ofint?

s.t.

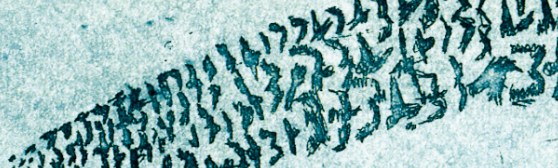

(1)

(n)

\title{
The role of carriage in the development of healthcare- associated infections with S. aureus and P. aeruginosa
}

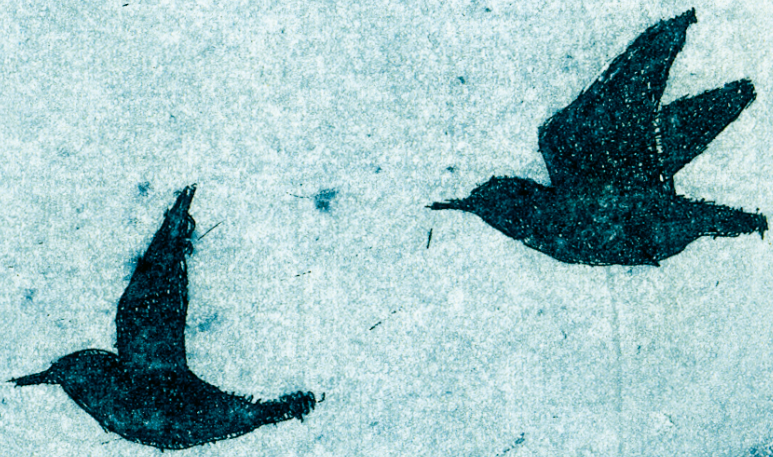

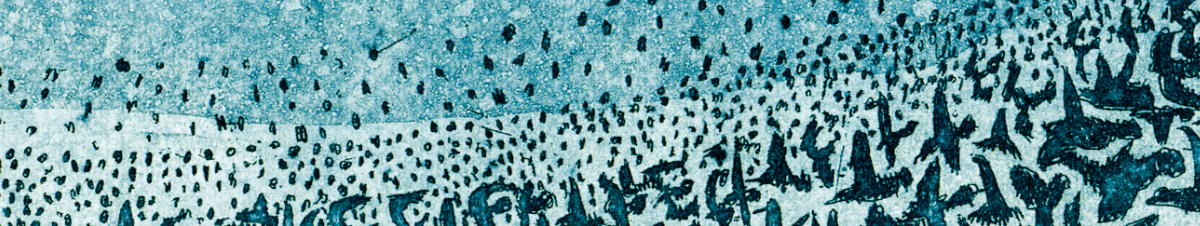
40,0 (s) 1 it 2 or An

Fleur Paling 



\section{The role of carriage in the development of healthcare-associated infections with $S$. aureus and $P$. aeruginosa}

Fleur Philine Paling 
Bekijk dit proefschrift in enkele minuten:

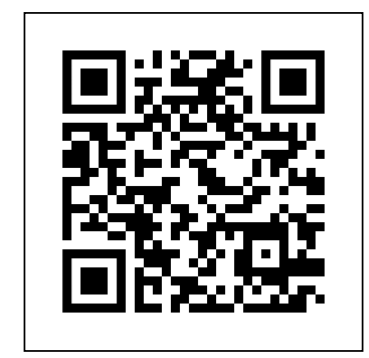

The role of carriage in the development of healthcare-associated infections with $S$. aureus and $P$. aeruginosa

ISBN/EAN: 978-90-393-7253-1

Copyright (C) 2020 F. P. Paling

All rights reserved. No part of this thesis may be reproduced, stored or transmitted in any way or by any means without the prior permission of the author, or when applicable, of the publishers of the scientific papers.

Cover design by Janis Goodman, Murmuration 2

Layout and design by Eduard Boxem, persoonlijkproefschrift.nl

Printed by Ipskamp Printing, proefschriften.net 


\section{The role of carriage in the development of healthcare-associated infections with $S$. aureus and $P$. aeruginosa}

Het aandeel van dragerschap in het ontwikkelen van gezondheidszorg-geassocieerde infecties met $S$. aureus en $P$. aeruginosa

(met een samenvatting in het Nederlands)

\section{Proefschrift}

ter verkrijging van de graad van doctor aan de Universiteit Utrecht

op gezag van de

rector magnificus, prof.dr. H.R.B.M. Kummeling, ingevolge het besluit van het college voor promoties

in het openbaar te verdedigen op

dinsdag 20 oktober 2020 des middags te 2.30 uur

door

\section{Fleur Philine Paling}

geboren op 28 augustus 1985

te Groningen 


\section{PROMOTOREN:}

Prof. dr. J.A.J.W. Kluytmans

Prof. dr. M.J.M. Bonten

Dit proefschrift werd (mede) mogelijk gemaakt met financiële steun van de SBOH. 


\section{TABLE OF CONTENTS}

Chapter 1 General introduction

PART ONE - RETROSPECTIVE

Chapter 2 S. aureus colonization at ICU admission as a risk factor for developing S. aureus ICU pneumonia

Chapter 3 Risk prediction for Staphylococcus aureus surgical site infection following cardiothoracic surgery; a secondary analysis of the V710P003 trial

Chapter 4 P. aeruginosa colonization at ICU admission as a risk factor for developing $P$. aeruginosa ICU pneumonia

PART TWO - PROSPECTIVE

Chapter 5 Rationale and design of ASPIRE-ICU: a prospective cohort study 72 on the incidence and predictors of Staphylococcus aureus and Pseudomonas aeruginosa pneumonia in the ICU

Chapter 6 Staphylococcus aureus colonization and the occurrence of ICU pneumonia; ASPIRE-ICU, a prospective international cohort study.

Chapter 7 Identifying patients at increased risk for S. aureus ICU pneumonia; results from an international prospective cohort study

PART THREE - CONCLUSIONS

Chapter 8 General discussion

Chapter 9 Appendix

Summary

Nederlandse samenvatting

Dankwoord

Curriculum Vitae

List of publications 


$$
\pi \pi
$$


General introduction

CHAPTER

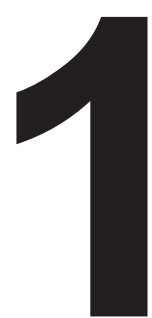


Each human being carries approximately 100 trillion bacteria in and on his/her body. Some say that we are inhabited with more bacterial cells than we are with human cells, although these estimates have substantial uncertainties. The aggregate of these (and all other) microorganisms in and on our body are called the human microbiota and includes on average $>1000$ different types of bacteria[1], [2]. Many of these do not cause any harm, a great deal of them are even essential for fundamental physiological processes. However, even though we know we cannot live without some of these coinhabitants, we do not nearly understand the role of all of them, not even are we aware of all of their presence.

Of some common resident microorganisms it is known that they have the ability to cause serious infections[2]. In this regard, Staphylococcus aureus and Pseudomonas aeruginosa are two very well-known examples, and a great body of evidence exists for both of them, describing numerous aspects of the interplay between human and bacterium[3]-[6]. Despite all knowledge it remains difficult to predict which person will get which infection and when this will occur. The research presented in this thesis intends to improve identification of these patients at risk.

\section{The usual suspects}

\section{Staphylococcus aureus}

To start with S. aureus, this truly is a pathogen in disguise. It is commonly carried on skin or anterior nares by many healthy people, not causing any harm. In some of us, the $S$. aureus are merely passing through every now and then, making the host an 'intermittent carrier'. Other people however, are more or less always carrying $S$. aureus in and on their body and are referred to as a 'persistent carrier'. There are reasons to believe that different carrier states relate to differences in disease risk[7] and the main study described in this thesis will generate longitudinal data on colonization status by that aiding to further substantiate or invalidate this hypothesis. Even so, the carriage rates described in this thesis are all resulting from cross-sectional assessments of colonization status, for reasons of availability as well as reproducibility in clinical practice. In the literature, these carriage rates range from 25-30 percent and differ per age group and body location[8], [9]. Furthermore, overall S. aureus carrier rates have decreased over the last decades, possibly due to improved personal hygiene and / or changes in socioeconomic status and family size [10].

Unfortunately, as already suggested in the previous paragraph, this colonizing resident is not only harmless. Although seeming so at first, S. aureus has the potential to quickly manifest into a serious or even life-threatening infection, needing invasive treatment. 
This can happen in case of weakening of the human defense system, e.g. after surgery, or when mechanically ventilated, but it can also happen in seemingly healthy people. The most important risk factor seems to be a break in the integrity of the skin. To complicate matters even further, not all carriers get infections once their defense decreases and also non-carriers can be victims of this pathogen[11], [12]. In this regard, prediction remains difficult. Nevertheless, knowledge of carrier status is an important step towards quantifying disease risk, as will be demonstrated in the following chapters of this thesis.

\section{Pseudomonas aeruginosa}

Whereas $S$. aureus is a very frequently carried bacterium, the carriage rates of $P$. aeruginosa are much lower. In ICU patients it was estimated to be around $10 \%$ or less [13]. Acquisition of this pathogen typically occurs after a patient has become ill, and from here subsequently progresses to serious infections. Examples are urogenital infections, but also pneumonia, especially in mechanically ventilated patients on an intensive care unit (ICU), is notorious. Once the infection is present, it is often difficult to treat due to multidrug resistance[14]. The association between prior colonization and subsequent infection is assumed, but compared to $S$. aureus, substantiated by less evidence. Furthermore, considering the lower rates of carriage, it will be necessary to also rely on other characteristics for the prediction of $P$. aeruginosa infections. In this thesis a first step will be taken towards this objective.

\section{Research network}

As can be imagined, infections caused by $S$. aureus and $P$. aeruginosa are frequently health-care associated and a major cause of morbidity as well as mortality, not to mention the subsequent financial burden[14]-[16]. The Combatting Bacterial Resistance in Europe groups (COMBACTE-NET for gram-positive infections and COMBACTEMAGNET for gram-negative infections) are 2 consortia that address, among other things, health-care associated infections (HAls) caused by $S$. aureus and $P$. aeruginosa. The consortia are a public-private partnership in which pharmaceutical companies work together with academic partners[17]. Having developed a network of hospitals (CLIN-Net) and associated laboratories (LAB-Net), that are available for the execution of clinical studies, this platform is ideal for assessing the research gaps described in the first paragraph, but reaches far beyond the scope of this thesis. 


\section{Aim and outline of this thesis}

All research results presented in this thesis were performed within the COMBACTE consortia described earlier, with the side note that the post-hoc analyses presented in the retrospective part (chapter 2-4) uses data that was collected outside of the COMBACTE groups.

In chapter $\mathbf{2}$ and chapter $\mathbf{4}$ we describe the analyses that were retrospectively performed on ICU cohorts of which the data was retrieved by searching the hospital network (CLIN-Net) of COMBACTE for existing databases eligible for our research question. Both analyses aimed to aid the design of the prospective study called ASPIRE-ICU (Advanced understanding of Staphylococcus aureus and Pseudomonas aeruginosa Infections in EuRopE - Intensive Care Units), which was conceptualized simultaneously, but executed after the results became available. In these chapters we assess the occurrence of ICU pneumonia caused by $S$. aureus and $P$. aeruginosa in three European hospitals as well as means to identify predisposing factors. The results described here, lead for example to using mechanical ventilation at ICU admission as an inclusion criterion for ASPIRE-ICU. More about the study design of ASPIRE-ICU, as well as the rationale behind it, can be found in chapter $\mathbf{5}$, where a summary of the study protocol is presented.

In the same way as in chapters 2 and 4 , in chapter 3 we intended to find predisposing factors for the development of $S$. aureus surgical site infection, another HAl, and quantify the share of prior $S$. aureus carriage in this regard. The data used for this analysis originated from an internationally executed vaccine study [18]. The subsequent observational cohort study (ASPIRE-SSI), which was designed partly using results of this analysis, is still ongoing, and the results will be described outside of this thesis.

The prospective part of this thesis discusses the first results from the main study, ASPIREICU. Chapter 6 addresses its primary objective, which is describing the epidemiology of $S$. aureus ICU pneumonia in relation to carriage of $S$. aureus, as well as other risk factors. In chapter $\mathbf{7}$ this culminates in a risk prediction model, aiming to identify those patients that are at highest risk of developing $S$. aureus ICU pneumonia.

Lastly, chapter $\mathbf{8}$ discusses the conclusions that can or cannot be drawn from all data present in this thesis, upcoming results and possible challenges for future research. 


\section{REFERENCES}

1. R. Sender, S. Fuchs, and R. Milo, "Revised Estimates for the Number of Human and Bacteria Cells in the Body," PLOS Biol., vol. 14, no. 8, p. e1002533, Aug. 2016.

2. M. J. B. Silva, M. B. H. Carneiro, B. dos Anjos Pultz, D. Pereira Silva, M. E. de M. Lopes, and L. M. dos Santos, "The multifaceted role of commensal microbiota in homeostasis and gastrointestinal diseases.," J. Immunol. Res., vol. 2015, p. 321241, 2015.

3. J. Kluytmans, A. van Belkum, and H. Verbrugh, "Nasal carriage of Staphylococcus aureus: epidemiology, underlying mechanisms, and associated risks.," Clin. Microbiol. Rev., vol. 10, no. 3, pp. 505-20, Jul. 1997.

4. M. Bassetti, A. Vena, A. Croxatto, E. Righi, and B. Guery, "How to manage Pseudomonas aeruginosa infections.," Drugs Context, vol. 7, p. 212527, 2018.

5. M. van Rijen, M. Bonten, R. Wenzel, and J. Kluytmans, "Mupirocin ointment for preventing Staphylococcus aureus infections in nasal carriers.," Cochrane database Syst. Rev., no. 4, p. CD006216, Jan. 2008.

6. L. Berra, J. Sampson, and J. Wiener-Kronish, "Pseudomonas aeruginosa: acute lung injury or ventilator-associated pneumonia?," Minerva Anestesiol., vol. 76, no. 10, pp. 824-32, Oct. 2010.

7. J. L. Nouwen, M. W. J. A. Fieren, S. Snijders, H. A. Verbrugh, and A. Van Belkum, "Persistent (not intermittent) nasal carriage ofStaphylococcus aureus is the determinant of CPD-related infections," Kidney Int., vol. 67, no. 3, pp. 1084-1092, Mar. 2005.

8. R. E. WILLIAMS, "Healthy carriage of Staphylococcus aureus: its prevalence and importance.," Bacteriol. Rev., vol. 27, pp. 56-71, Mar. 1963.

9. J. Kluytmans, A. van Belkum, and H. Verbrugh, "Nasal carriage of Staphylococcus aureus: epidemiology, underlying mechanisms, and associated risks.," Clin. Microbiol. Rev., vol. 10, no. 3, pp. 505-20, Jul. 1997.

10. H. F. L. Wertheim et al., "The role of nasal carriage in Staphylococcus aureus infections.," Lancet Infect. Dis., vol. 5, no. 12, pp. 751-62, Dec. 2005.

11. P. Berthelot et al., "Is nasal carriage of Staphylococcus aureus the main acquisition pathway for surgical-site infection in orthopaedic surgery?," Eur. J. Clin. Microbiol. Infect. Dis., vol. 29, no. 4, pp. 373-82, Apr. 2010. 
12. C. von Eiff, K. Becker, K. Machka, H. Stammer, and G. Peters, "Nasal carriage as a source of Staphylococcus aureus bacteremia. Study Group.," N. Engl. J. Med., vol. 344, no. 1, pp. 11-6, Jan. 2001.

13. A. D. Harris et al., "Pseudomonas aeruginosa Colonization in the Intensive Care Unit: Prevalence, Risk Factors, and Clinical Outcomes," Infect. Control Hosp. Epidemiol., vol. 37, no. 5, pp. 544-548, May 2016.

14. J. Walter et al., "Healthcare-associated pneumonia in acute care hospitals in European Union/ European Economic Area countries: an analysis of data from a point prevalence survey, 2011 to 2012," Eurosurveillance, vol. 23, no. 32, Aug. 2018.

15. C. Suetens et al., "Prevalence of healthcare-associated infections, estimated incidence and composite antimicrobial resistance index in acute care hospitals and long-term care facilities: results from two European point prevalence surveys, 2016 to 2017.," Euro Surveill., vol. 23, no. 46, 2018.

16. S. S. Magill et al., "Multistate Point-Prevalence Survey of Health Care-Associated Infections," N. Engl. J. Med., vol. 370, no. 13, pp. 1198-1208, Mar. 2014.

17. T. Kostyanev et al., "The Innovative Medicines Initiative's New Drugs for Bad Bugs programme: European public-private partnerships for the development of new strategies to tackle antibiotic resistance.," J. Antimicrob. Chemother., vol. 71, no. 2, pp. 290-5, Feb. 2016.

18. V. G. Fowler et al., "Effect of an investigational vaccine for preventing Staphylococcus aureus infections after cardiothoracic surgery: a randomized trial.," JAMA, vol. 309, no. 13, pp. 1368-78, Apr. 2013. 



\section{PART ONE}

RETROSPECTIVE 


$$
\pi \pi
$$




\title{
S. aureus colonization at ICU admission as a risk factor for developing $S$. aureus ICU pneumonia
}

\author{
Fleur P. Paling ${ }^{1}$ \\ Martin Wolkewitz ${ }^{2}$ \\ Lonneke G.M. Bode ${ }^{3}$ \\ Peter M.C. Klein Klouwenberg ${ }^{3,4}$ \\ David S.Y. Ong ${ }^{3,4}$ \\ Pieter Depuydt ${ }^{5}$ \\ Liesbet de Bus ${ }^{5}$ \\ Frangiscos Sifakis ${ }^{6}$ \\ Marc J.M. Bonten ${ }^{1,3}$ \\ Jan A.J.W. Kluytmans ${ }^{1}$
}

Adapted from: Clinical Microbiology and Infection, 23(1)

2017. https://doi.org/10.1016/j.cmi.2016.09.022

\footnotetext{
${ }^{1}$ Julius Center for Health Sciences and Primary Care, University Medical Center Utrecht, Utrecht, Netherlands

${ }^{2}$ Institute for Medical Biometry and Statistics, University Medical Center Freiburg, Freiburg, Germany

${ }^{3}$ Department of Medical Microbiology, University Medical Center Utrecht, Utrecht, Netherlands

${ }^{4}$ Department of Intensive Care Medicine, University Medical Center Utrecht, Utrecht, Netherlands

${ }^{5}$ Department of Intensive Care Medicine, University Hospital of Ghent, Ghent, Belgium

${ }^{6}$ AstraZeneca LP, Gaithersburg, Maryland, U.S.A.
} 


\section{ABSTRACT}

Objective: To quantify the incidence of intensive care unit (ICU) acquired pneumonia caused by Staphylococcus aureus (S. aureus) and its association with S. aureus colonization at ICU admission.

Methods: This was a post-hoc analysis of two cohort studies in critically ill patients. The primary outcome was the incidence of microbiologically confirmed S. aureus ICUacquired pneumonia. Incidences of $S$. aureus ICU pneumonia and associations with $S$. aureus colonization at ICU admission were determined using competing risks analyses. In all ICUs, patients were screened for respiratory tract $S$. aureus carriage on admission as part of infection control policies. Pooling of data was deemed not possible due to heterogeneity in baseline differences in patient population.

Results: The two cohort studies contained data of 9,156 ICU patients. The average carriage rate of $S$. aureus among screened patients was $12.7 \%$. In total, 1,185 (12.9\%) patients developed ICU pneumonia. Incidences of S. aureus ICU pneumonia were $1.33 \%$ and $1.08 \%$ in cohorts 1 and 2, respectively. After accounting for competing events, the adjusted subdistribution hazard ratio (SHR) of S. aureus colonization at admission for developing S. aureus ICU pneumonia was 9.55, (95\% confidence interval [CI] 5.31-17.18) in cohort 1 and $14.54(95 \% \mathrm{Cl} 7.24-29.21)$ in cohort 2.

Conclusion: The overall cumulative incidence of S. aureus ICU pneumonia in these ICUs was low. Patients colonized with S. aureus at ICU admission had an up to 15 times increased risk for developing this outcome compared to non-colonized patients. 


\section{INTRODUCTION}

Pneumonia acquired during treatment in the Intensive Care Unit (ICU), or ICU-acquired pneumonia, causes considerable morbidity and mortality, and contributes significantly to the financial burden of the healthcare system[1]. The epidemiology of ICU pneumonia, including ventilator-associated pneumonia (VAP), has not been fully described. This is in part due to variations in case definitions and surveillance systems utilized in different settings. Efforts to standardize assessments of disease measures and definitions in hospitals across different countries are hampered by temporal and geographic variation in disease risk [2, 3].

ICU pneumonia is frequently caused by Staphylococcus aureus, which is a human commensal and a frequent colonizer in healthy people. However, even seemingly 'innocent' and antibiotic-susceptible isolates frequently cause life-threatening infections in high-risk patients[4]. Colonization occurs most frequently in the nose, but other sites have been identified, e.g. the pharynx, perineum, or other parts of the skin. Cross-sectional colonization rates are on average around $25-30 \%$ in the general adult population. Longitudinal studies describe three patterns of carriage: persistent, intermittent, and non-carriers[5]. Associations between S. aureus colonization and staphylococcal disease have been demonstrated repeatedly [5-7] and eradication interventions reduced post-surgical infection rates $[8,9]$.

The aim of the current study was to systematically assess the impact of $S$. aureus colonization at the time of ICU admission on the incidence of ICU-acquired S. aureus pneumonia. Identifying the patient populations at risk for developing S. aureus ICU pneumonia is important to support effective interventions that aim to prevent $S$. aureus infections. 


\section{METHODS}

\section{Study design and patient population}

We used data of two independent, prospectively collected observational cohort studies. The independent medical ethics committee in participating countries waived the need for both informed consent and full ethical review of this post-hoc analysis.

One cohort study contained information from two tertiary hospitals in the Netherlands (cohort 1), from January 2011 until December 2013, collected for the MARS (Molecular Diagnosis and Risk Stratification of Sepsis) project[10]. The other study was performed in a tertiary hospital in Belgium (cohort 2), where data on epidemiology of ICU-acquired infections were collected, from January 2010 until June 2014, by means of the locally developed COSARA software application which allows a continuous prospective registration of all infection- and antibiotic-related data[11]. Both databases contained data from mixed ICU populations and were initially developed as biobanks with a view to future medical research of unknown nature at the time of sampling and data collection. The databases were retrieved through the Clin-NET network of hospitals, which is a developing network of European COMBACTE hospitals to be used for clinical trials of antimicrobials $[12,13]$.

Subjects with a length of stay (LOS) of $\geq 48 \mathrm{~h}$ were regarded as the patient population at risk for the primary outcome of ICU pneumonia. For the outcome VAP the patient population at risk was defined as subjects with LOS $\geq 48 \mathrm{~h}$ and ever on mechanical ventilation (MV). All patients in cohort 1 with an expected LOS in ICU of $\geq 48$ hours or expected duration of $M V$ of $\geq 24$ hours had received prophylactic systemic antibiotics (selective digestive tract decontamination [SDD]) [20], which included antibiotics directed at $S$. aureus. In comparison, chlorhexidine body washes were used routinely on all patients in cohort 2 , which also target $S$. aureus.

\section{Definition of $S$. aureus colonization}

In cohort 1 routine endotracheal aspirate (ETA)/sputum and rectal screening was performed in all patients receiving SDD (e.g. expected LOS $\geq 48 \mathrm{~h}$ or expected MV duration of $\geq 24 \mathrm{~h}$ ); in cohort 2 routine ETA/sputum and rectal screening was performed in all patients with an expected LOS of $\geq 48 \mathrm{~h}$. No enrichment plates were used for culture of $S$. aureus. Patients were regarded as $S$. aureus colonized at ICU admission if S. aureus was cultured from nasopharynx/sputum/skin/bronchoalveolar lavage on the day of ICU admission (or 2 days before or after) and if there was no S. aureus infection 
diagnosed on these days. If a patient was not screened it was coded as 'unknown colonization status'.

\section{Outcome of interest}

Our primary outcome of interest is the incidence of all episodes of $S$. aureus ICU pneumonia occurring $\geq 48$ hours after ICU admission. Patients with (hospital-acquired) pneumonia $\leq 48$ hours of ICU admission were not excluded from the analysis, as they were still at risk to develop new ICU pneumonia. Confirmed endpoints are those with ICU pneumonia and laboratory isolation of $S$. aureus from any location in the lower respiratory tract.

Our secondary outcome is the incidence of $S$. aureus pneumonia occurring $\geq 48$ hours after start of MV (including $\leq 48$ hours after weaning), defined as VAP. In cohort 1, ICU pneumonia was defined on radiologic criteria and one or more clinical sign/symptom or laboratory parameter, such as cough, fever, elevated CRP or leukocyte count. In cohort 2 the definitions were based on radiologic criteria in combination with at least one or more clinical or laboratory criteria. In both sites there was a cross-validation of the assigned diagnoses by a research physician of the project.

\section{Statistical analysis}

Two separate analyses were performed; one evaluating the incidence density of $S$. aureus ICU pneumonia, calculated depending on time from admission; and one evaluating the incidence density of $S$. aureus VAP, calculated depending on time from ventilation. A competing risks analysis was performed. This is a special type of Cox survival analysis that allows controlling for events that are 'competing' with the event of interest and thus have their effects on the observed risks. In our case, for example, if a certain exposure status is associated with a prolonged or shortened stay in ICU (in other words, if it is associated with one of the competing events), this will have its effect on the absolute cumulative risk of acquiring our event of interest.[14, 15]

For our analysis, ICU discharge/death without ICU pneumonia (/VAP) were considered to be competing events for the outcome ICU pneumonia or VAP, respectively (see figure 1). Cause-specific hazards were calculated for each event, which can be interpreted as the daily 'risk' of observing that specific event. Each day that a patient is in the ICU the patient is exposed to these cause-specific hazards, which are 'pulling' the patient towards a certain event. From this, cause-specific hazard ratios were calculated to comp are the separate exposure statuses. In a second step, subdistribution hazard ratios were calculated to draw conclusions about cumulative risks; they can be interpreted 
as a comparison of the cumulative incidence functions, which in their turn describe how the absolute risk of infection is developing during the at-risk time in the ICU, while accounting for competing events.
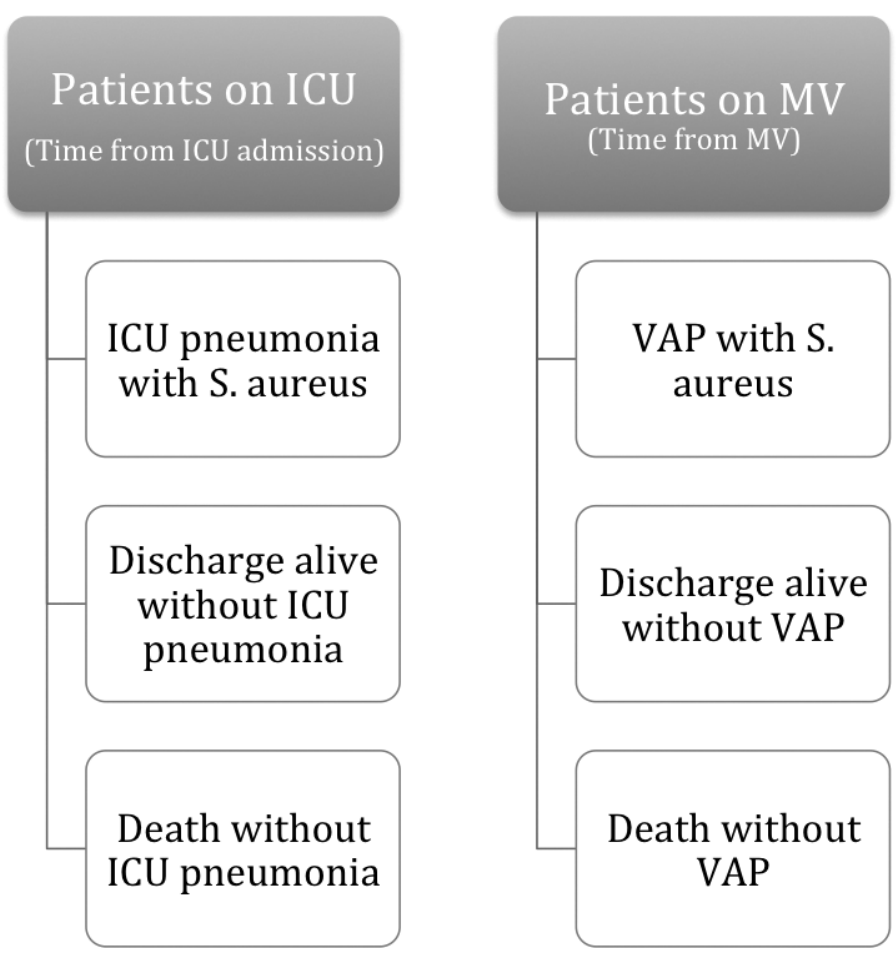

Figure 1. Competing risks models for ICU pneumonia (left model) and VAP (right model). ICU, intensive care unit; VAP, ventilator associated pneumonia; MV, mechanical ventilation.

\section{Model building}

Considering the method of capturing outcome in the database and differences in casemix, of which the latter will be discussed in more detail later, the statistical analyses could only be performed separately for each cohort.

A Fine \& Gray model was fitted, covariables being added to the model based on literature and clinical reasoning based on literature and clinical reasoning, abiding by the rule of thumb of 1 covariate per 10 events. Statistical analyses were performed using SAS version 9.2 and $R$ version 2.10.00. Included covariables in the models assessing ICU pneumonia were colonization at admission, MV at admission, ICU admission type (medical vs. surgical), age (continuous variable), Acute Physiological and Chronic Health 
Evaluation (APACHE) IV score[16] (cohort 1 only, continuous variable) and gender (cohort 2 only).

Table 1. Baseline characteristics of full cohorts

\begin{tabular}{lcc}
\hline & $\begin{array}{c}\text { Cohort 1 } \\
\mathbf{N}=4063\end{array}$ & $\begin{array}{c}\text { Cohort 2 } \\
\mathbf{N}=5092\end{array}$ \\
& \multicolumn{1}{c}{$N$ (\%) or mean (SD) } \\
\hline Gender: male & $2502(61.6)$ & $3178(62.4)$ \\
Age & $59.3(15.9)$ & $59.4(16.1)$ \\
Length of stay in days & $9.2(11.6)$ & $9.1(11.7)$ \\
Median & 6 & 5 \\
Surgical admission & $1599(39.4)$ & $2917(57.3)$ \\
APACHE IV score ${ }^{\dagger}$ & $76(29)$ & $N A(N A)$ \\
Colonization status ${ }^{\dagger}$ & & $314(6.2)$ \\
- S. aureus positive & $399(9.8)$ & $2133(41.9)$ \\
- S. aureus negative & $2753(67.8)$ & $2645(51.9)$ \\
- Unknown / missing & $911(22.4)$ & $691(13.6)$ \\
ICU mortality & $656(16.2)$ & $2591(50.9)$ \\
Mechanical ventilation ${ }^{\dagger}$ & $3356(82.6)$ & $675(13.25)$ \\
ICU pneumonia & $510(12.55)$ & $55(1.08)$ \\
S. aureus & $54(1.33)$ & $360(7.07)$ \\
Other pathogen & $344(8.47)$ & $260(5.11)$ \\
Unknown / missing pathogen & $112(2.76)$ & \\
\hline
\end{tabular}

+ measured at ICU admission

$\mathrm{SD}=$ standard deviation, APACHE=Acute Physiology \& Chronic Health Evaluation, ICU=Intensive Care Unit Number of VAP cases are not included here, considering that they come from a different baseline population (only patients on mechanical ventilation)

\section{RESULTS}

\section{Patient population and incidence of ICU pneumonia/VAP}

Together, both cohort studies contained information on 9,155 patients $(84,002$ patient days); 4,063 and 5,092 in cohort 1 and 2, respectively (see Table 1 for baseline characteristics). Cohort 2 had a higher proportion of surgical admissions and lower proportion of patients with MV on admission. S. aureus colonization status was assessed in $80 \%$ and $58 \%$ of the patients, the average carriage rate being $12.7 \%$, which was mostly based on lower respiratory tract samples (in cohort 1 and 2, 61\% and 58\%, respectively). In cohort 1 , routine nasopharyngeal/oral swabs were taken in $41 \%$ as part of SDD, which were positive for S. aureus in $9 \%$. In cohort 2, nose swabs were 
positive for S. aureus in $33 \%$, but were only done in $10 \%$ of the patients, as this was not performed routinely.

In total, 1,185 (12.9\%) developed ICU pneumonia. Of these pneumonias, 9.2\% were caused by $S$. aureus ( 10.6 and $8.1 \%$ in cohort 1 and 2 respectively). This corresponds to an incidence proportion of S. aureus ICU pneumonia of 1.33 and $1.08 \%$ in cohort 1 and 2, respectively. Within S. aureus colonized patients, the incidence proportion of S. aureus ICU pneumonia was 7.27 and $8.28 \%$ respectively (supplementary Table 6). The median time from ICU admission to $S$. aureus ICU pneumonia was 6 days for both cohorts.

For the VAP analyses we had information available on a total of 6,736 patients $(73,217$ patient days); 3,801 and 2,935 in cohort 1 and 2, respectively (see supplementary Table 1 for baseline characteristics). The incidence proportion of $S$. aureus VAP was 1.08 and $1.43 \%$ in cohort 1 and 2 , respectively (Table 2 ). Within $S$. aureus colonized patients, the incidence proportion of $S$. aureus VAP was 5.40 and $9.72 \%$ in cohort 1 and 2 respectively (supplementary Table 7).

Table 2. Incidence proportions of ventilator associated pneumonia (VAP)

\begin{tabular}{lcc}
\hline & Cohort $\mathbf{1}(\mathbf{n}=\mathbf{3}, \mathbf{8 0 1 *})$ & Cohort 2 (n=2,935*) \\
& $N(\%)$ & $N(\%)$ \\
\hline Ventilator-associated pneumonia & $\mathbf{3 5 2 ( 9 . 2 9 )}$ & $\mathbf{4 1 0 ( 1 3 . 9 7 )}$ \\
S. aureus & $41(1.08)$ & $42(1.43)$ \\
Other pathogen & $263(6.94)$ & $245(8.35)$ \\
Unknown / missing pathogen & $48(1.27)$ & $123(4.19)$ \\
\hline
\end{tabular}

* Please note that for this analysis a subgroup of patients was used from both cohorts: only patients ever on mechanical ventilation were included. For baseline characteristics of this subgroup, see supplementary Table 1. 


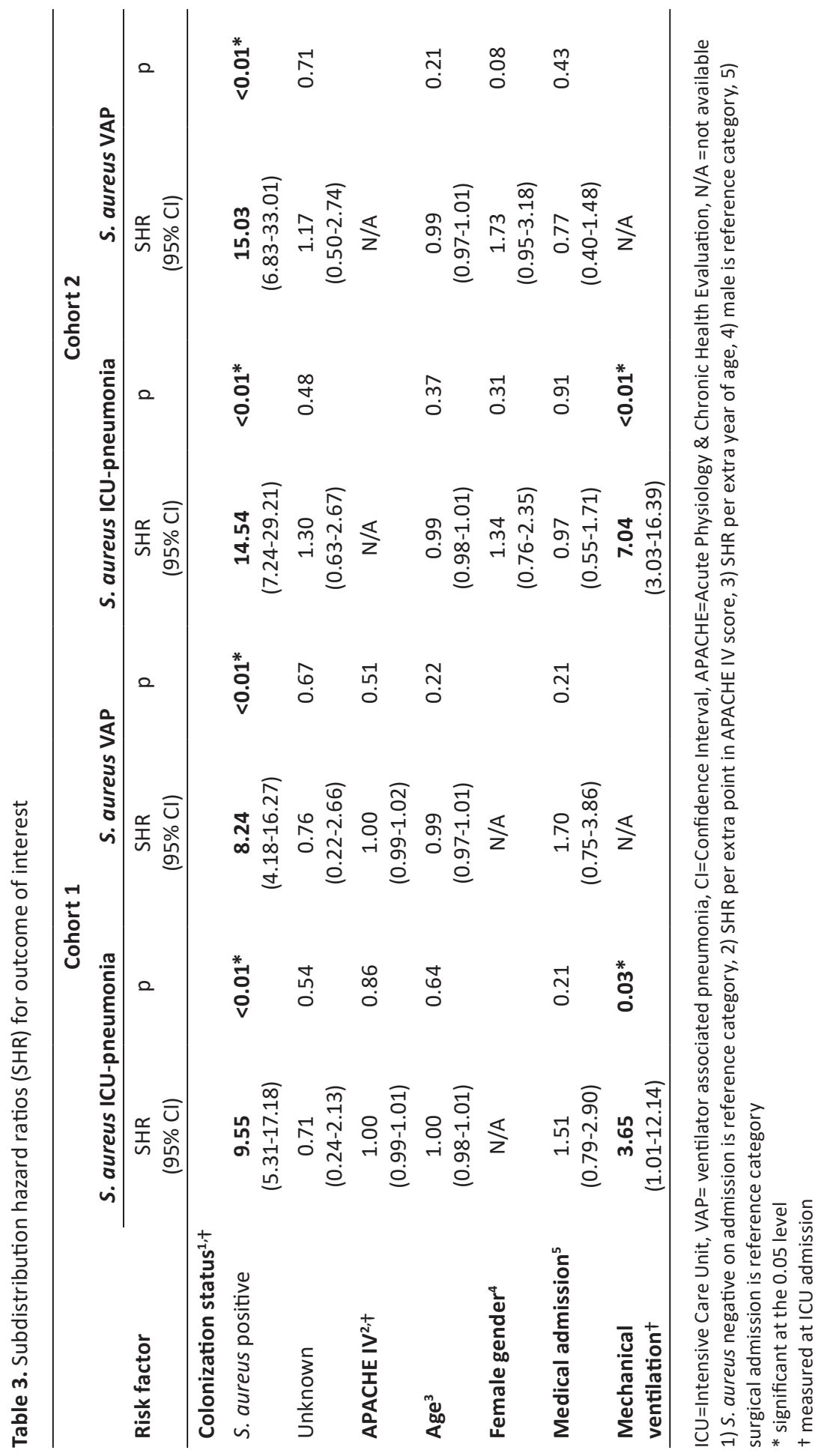




\section{Cause-specific and subdistribution hazard ratios - cohort 1}

Cause-specific hazard ratios (CSHRs) for developing S. aureus ICU pneumonia or VAP for patients colonized at ICU admission were 11.05 and 9.41, respectively $(p<0.001)$, compared to non-colonized patients. See supplementary Tables 2 and 3 for detailed information on all CSHRs.

After accounting for competing events, S. aureus colonization at ICU admission was still a risk factor for developing S. aureus ICU pneumonia or VAP: a subdistribution hazard ratio (SHR) of 9.55 and 8.24 was found respectively $(p<0.001)$. This can be interpreted as follows: on average the cumulative incidence function for developing $S$. aureus ICU pneumonia is 9.55 times higher than the cumulative incidence function for a noncolonized ICU patient (see also figure 2). Mechanical ventilation at ICU admission was found to be a risk factor for S. aureus ICU pneumonia with a SHR of $3.65(p=0.03)$ for patients with MV on admission (Table 3).

\section{Cause-specific and subdistribution hazard ratios - cohort 2}

S. aureus colonization status at ICU admission was a risk factor for the development of both S. aureus ICU pneumonia and VAP with CSHRs of 15.15 and 15.84 respectively $(p<0.001)$. See supplementary Tables 4 and 5 for detailed information on all CSHRs.

Similarly as in cohort 1 , after accounting for competing events, S. aureus colonization at ICU admission was still a risk factor for the development of $S$. aureus ICU-pneumonia and VAP (SHR 14.54 and 15.03, p<0.001), as was MV at ICU admission for S. aureus ICU pneumonia (SHR 7.04, p<0.001, table 3). The cumulative incidence functions, describing the development of risk of infection during ICU stay can be found in figure 2 .

\section{DISCUSSION}

This study showed an incidence proportion of $S$. aureus ICU pneumonia between 1.1 and $1.3 \%$ in ICU patients with a LOS of $\geq 48$ hours. Hazard ratios for $S$. aureus colonized patients compared to non-colonized were up to 14.5 ; , meaning that colonized patients roughly have a 15 times higher chance of developing SA ICU pneumonia throughout their ICU stay than non-colonized. In many studies, associations between S. aureus carriage and S. aureus infection have been reported[5-7]. However, studies investigating the association between respiratory tract colonization with any $S$. aureus at ICU admission and S. aureus VAP or ICU pneumonia are few $[17,18,19]$; and while these studies reported an increased risk of $S$. aureus disease for carriers of $S$. aureus, most did not perform multivariate analyses or a competing risks analysis taking into account 
a) S. aureus ICU pneumonia, cohort 1

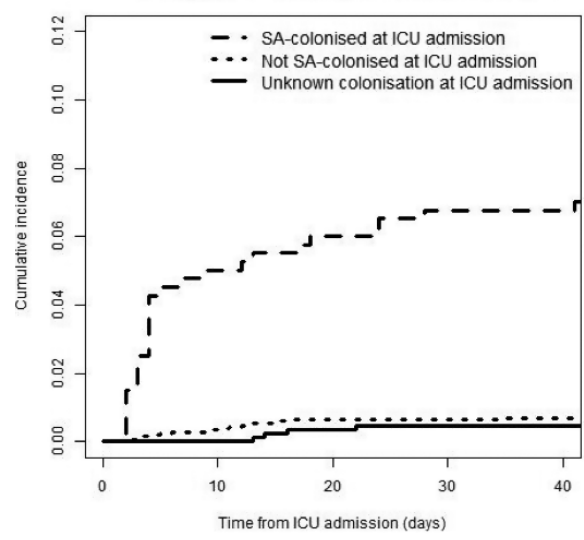

c) S. aureus ICU pneumonia, cohort 2

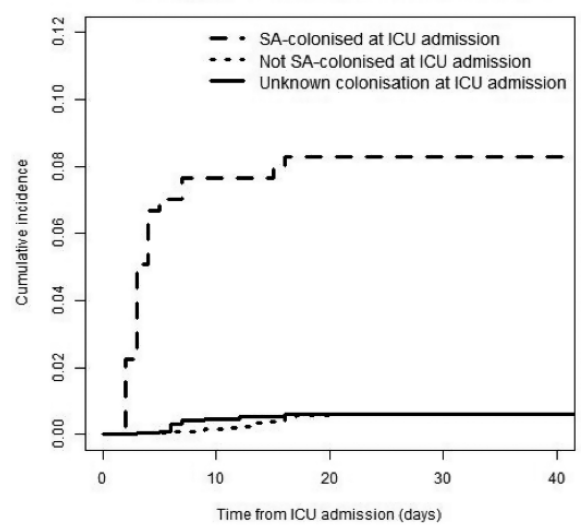

b) S. aureus VAP, cohort 1

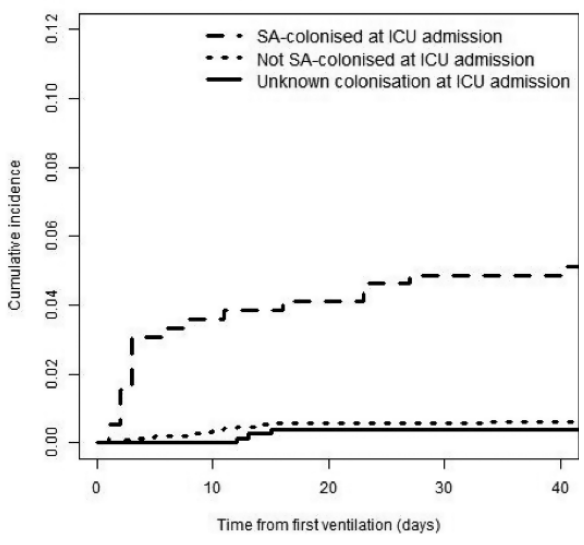

d) S. aureus VAP, cohort 2

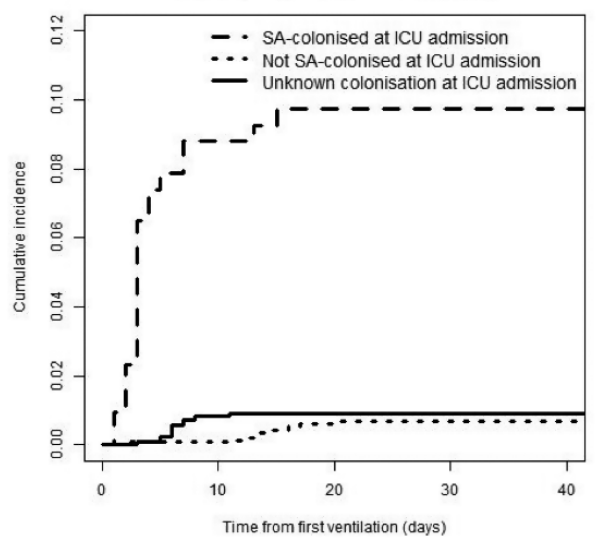

Figure 2. Cumulative incidence functions. (a) $S$. aureus ICU pneumonia, cohort 1. (b) S. aureus VAP, cohort 1. (c) S. aureus ICU pneumonia, cohort 2. (d) S. aureus VAP, cohort 2. ICU, intensive care unit; VAP, ventilator associated pneumonia.

the competing events (e.g. ICU discharge and death without S. aureus ICU pneumonia / VAP).. In the setting of an ICU, with competing events present, this could result in biased estimates [14]. Understanding the true impact of carriage (and other risk factors) on development of S. aureus ICU pneumonia is important for the identification of the patient population that will benefit the most from preventive interventions that may become available in the future. 
Two cohort studies from university hospitals were selected for this post-hoc analysis. Even though the studies were performed in neighbouring countries, there were differences between their ICU populations. The case-mix in cohort 2 contained more unventilated and surgical patients at baseline, suggesting a relatively healthier population. Unfortunately, APACHE scores were not available in the database of cohort 2, thus it was not possible to use a standardized scoring system to confirm this assumption. However, the finding that screening for colonization at baseline was performed in $48 \%$ of cohort 2, compared to $78 \%$ of cohort 1 may support the assumption that patients in cohort 2 were healthier, considering that in both cohorts, screening is done in patients with an expected LOS of $\geq 48 \mathrm{~h}$.

Differences in case-mix, local practices and disease severity may have resulted in different background risks for the outcomes of interest, and thus have influenced the interpretation of the calculated incidence of ICU pneumonia as a whole. All patients in cohort 1 with an expected LOS in ICU of $\geq 48$ hours had received prophylactic systemic antibiotics (selective digestive tract decontamination) [20], which included antibiotics directed at S. aureus. In comparison, chlorhexidine body washes were used routinely on all patients in cohort 2 , which also targets $S$. aureus, but in a different manner.

Furthermore, it is questionable whether the definition of outcome was the same across the two sites; even though radiologic criteria in combination with one or more clinical/ laboratory signs were used to diagnose ICU pneumonia at both sites, and dedicated researchers cross-validated all diagnoses. One could argue that the definitions were at least comparable, despite not being standardized. In general the definition of ICU pneumonia, and especially VAP, is a topic upon which much discussion exists. While a standardized definition has been proposed, the standard requires burdensome diagnostics [21]. Until now, there are no reliable, non-invasive tests available that have a satisfactory sensitivity and specificity and positive predictive value. $[2,3]$ to verify the proposed definitions; and for this reason the definitions used at the two sites were the best available for this analysis.

Interestingly, we found that the percentage of ICU pneumonias and VAPs without a causative pathogen was somewhat higher in cohort 2, even though all long-stay ICU patients in cohort 1 were routinely given prophylactic antibiotics targeting $S$. aureus. It is unclear whether this is due to a difference in (antibiotic) management, culture frequency or colony selection for species determination in the laboratory.

One may argue that those who were known to be colonized with $S$. aureus were possibly more often diagnosed with a $S$. aureus ICU pneumonia or VAP, resulting in a 
positive association. However, only culture-proven S. aureus outcome were considered for this analysis; and an association of this magnitude is unlikely to be explained by prior knowledge on colonization status alone. Some ICU pneumonias or VAPs with an unknown causative pathogen could have been caused by non-cultured $S$. aureus. To assess if there were any trends to support this hypothesis, we performed a sensitivity analysis, also taking into account ICU pneumonia with any other confirmed pathogen and ICU pneumonia with an unknown pathogen as competing events, while prioritizing S. aureus ICU pneumonia. We did not find such a trend.

In summary, there are large differences in case-mix, laboratory- and clinical management, and minor differences in the definition of outcome. The difference in case-mix makes comparison of crude incidences especially difficult, since adjustment for disease severity was not possible; however, findings from both cohorts were comparable, which may suggest generalizability of the results across these two ICU centers. Easier to interpret are the cumulative incidence functions. When comparing these, they clearly show an increased risk of the outcome for colonized compared to non-colonized patients during the at-risk time in ICU.

An important lesson that can be drawn from this study is that performing post-hoc studies, including merging of databases, is cumbersome due to limited availability and differences in surveillance methods, case-mix of patients and outcome definitions. It underscores the need for, at the least, a more universal definition of (ICU) pneumonia, but at the best, a multinational systematic surveillance system that systematically collects de-identified individual patient data; and use of advanced statistical methods to control for competing risks in the ICU patient population to better identify high risk patients.

\section{CONCLUSION}

The overall incidence proportion of S. aureus ICU pneumonia was relatively low (1.11.3\%). Patients colonized at ICU admission with S. aureus had a 10-15 times increased hazard for developing S. aureus ICU pneumonia, compared to non-colonized patients. For S. aureus VAP, incidences were similar (1.1-1.4\%), as well as the hazard ratios for $S$. aureus colonized vs. non-colonized (8.4-15.0). Interventions should consider targeting this high-risk population. 
Acknowledgements: Results were previously presented at ECCMID 2015 in Copenhagen, Denmark.

Funding: This research project receives support from the Innovative Medicines Initiative Joint Undertaking under grant agreement no. 115523/ 115620/ 115737 resources of which are composed of financial contribution from the European Union Seventh Framework Program (FP7/2007-2013) and EFPIA companies in kind contribution. 


\section{REFERENCES}

1. Safdar N, Dezfulian C, Collard HR, Saint S (2005) Clinical and economic consequences of ventilator-associated pneumonia: a systematic review. Crit Care Med 33:2184-93.

2. Ottosen J, Evans $H$ (2014) Pneumonia: challenges in the definition, diagnosis, and management of disease. Surg Clin North Am 94:1305-17. doi: 10.1016/j.suc.2014.09.001

3. Nair GB, Niederman MS (2015) Ventilator-associated pneumonia: present understanding and ongoing debates. Intensive Care Med 41:34-48. doi: 10.1007/s00134-014-3564-5

4. Lowy FD (1998) Staphylococcus aureus infections. N Engl J Med 339:520-32. doi: 10.1056/ NEJM199808203390806

5. Wertheim HFL, Melles DC, Vos MC, et al. (2005) The role of nasal carriage in Staphylococcus aureus infections. Lancet Infect Dis 5:751-62. doi: 10.1016/S1473-3099(05)70295-4

6. Pujol M, Peña C, Pallares R, et al. (1996) Nosocomial Staphylococcus aureus bacteremia among nasal carriers of methicillin-resistant and methicillin-susceptible strains. Am J Med 100:509-16.

7. Von Eiff C, Becker K, Machka K, et al. (2001) Nasal carriage as a source of Staphylococcus aureus bacteremia. Study Group. N Engl J Med 344:11-6. doi: 10.1056/NEJM200101043440102

8. Bode LGM, Kluytmans JAJW, Wertheim HFL, et al. (2010) Preventing surgical-site infections in nasal carriers of Staphylococcus aureus. N Engl J Med 362:9-17. doi: 10.1056/NEJMoa0808939

9. Van Rijen M, Bonten M, Wenzel R, Kluytmans J (2008) Mupirocin ointment for preventing Staphylococcus aureus infections in nasal carriers. Cochrane database Syst Rev CD006216. doi: 10.1002/14651858.CD006216.pub2

10. Klein Klouwenberg PMC, Ong DSY, Bos LDJ, et al. (2013) Interobserver agreement of Centers for Disease Control and Prevention criteria for classifying infections in critically ill patients. Crit Care Med 41:2373-8. doi: 10.1097/CCM.0b013e3182923712

11. De Bus L, Diet G, Gadeyne B, et al. (2014) Validity analysis of a unique infection surveillance system in the intensive care unit by analysis of a data warehouse built through a workflowintegrated software application. J Hosp Infect 87:159-64. doi: 10.1016/j.jhin.2014.03.010

12. Rex JH (2014) ND4BB: addressing the antimicrobial resistance crisis. Nat Rev Microbiol 12:231-232. doi: 10.1038/nrmicro3245

13. Combatting Bacterial Resistance in Europe. www.combacte.com. Accessed September 15, 2015 
14. Wolkewitz M, Vonberg RP, Grundmann H, et al. (2008) Risk factors for the development of nosocomial pneumonia and mortality on intensive care units: application of competing risks models. Crit Care 12:R44. doi: 10.1186/cc6852

15. Wolkewitz M, Cooper BS, Bonten MJM, et al. (2014) Interpreting and comparing risks in the presence of competing events. BMJ 349:g5060.

16. Zimmerman JE, Kramer AA, McNair DS, Malila FM (2006) Acute Physiology and Chronic Health Evaluation (APACHE) IV: hospital mortality assessment for today's critically ill patients. Crit Care Med 34:1297-310. doi: 10.1097/01.CCM.0000215112.84523.F0

17. Corbella X, Domínguez MA, Pujol M, et al. (1997) Staphylococcus aureus nasal carriage as a marker for subsequent staphylococcal infections in intensive care unit patients. Eur J Clin Microbiol Infect Dis 16:351-7.

18. Rocha LA, Marques Ribas R, da Costa Darini AL, Gontijo Filho PP (2013) Relationship between nasal colonization and ventilator-associated pneumonia and the role of the environment in transmission of Staphylococcus aureus in intensive care units. Am J Infect Control 41:123640. doi: 10.1016/j.ajic.2013.04.009

19. Garrouste-Orgeas M, Timsit JF, Kallel H, et al. (2001) Colonization with methicillin-resistant Staphylococcus aureus in ICU patients: morbidity, mortality, and glycopeptide use. Infect Control Hosp Epidemiol 22:687-92. doi: 10.1086/501846

20. De Smet AMGA, Kluytmans JAJW, Cooper BS, et al. (2009) Decontamination of the digestive tract and oropharynx in ICU patients. N EngI J Med 360:20-31. doi: 10.1056/NEJMoa0800394

21. Centers for Disease Control (2014) Ventilator-Associated Pneumonia ( VAP ) Event. http:// www.cdc.gov/nhsn/pdfs/pscmanual/6pscvapcurrent.pdf. Accessed September 15, 2015 


\section{SUPPLEMENTARY MATERIAL}

Supplementary Table 1: Baseline characteristics of subgroup at risk for ventilator associated pneumonia

Supplementary Table 2: Cause-specific hazard ratios (CSHR) per competing event for S. aureus ICU pneumonia in cohort 1

Supplementary Table 3: Cause-specific hazard ratios (CSHR) per competing event for S. aureus VAP in cohort 1

Supplementary Table 4: Cause-specific hazard ratios (CSHR) competing event for S. aureus ICU pneumonia cohort 2

Supplementary Table 5: Cause-specific hazard ratios (CSHR) per competing event for S. aureus VAP in cohort 2

Supplementary Table 6: Incidence proportions of ICU pneumonia in patients colonized with $S$. aureus at ICU admission

Supplementary Table 7: Incidence proportions of ventilator-associated pneumonia in patients colonized with S. aureus at ICU admission

Supplementary Table 8: Cumulative incidences (absolute and \%) of primary and secondary outcome, grouped by $S$. aureus colonization status at ICU admission. 
Supplementary Table 1. Baseline characteristics of subgroup at risk for ventilator associated pneumonia

\begin{tabular}{|c|c|c|}
\hline & $\begin{array}{l}\text { Cohort } 1 \\
\mathrm{~N}=3,801\end{array}$ & $\begin{array}{l}\text { Cohort } 2 \\
\mathrm{~N}=2,935\end{array}$ \\
\hline & \multicolumn{2}{|c|}{$N(\%)$ or mean $(S D)$} \\
\hline Gender: male & $2366(62.3)$ & $1858(63.3)$ \\
\hline Age & $59.3(16.0)$ & $59.9(15.8)$ \\
\hline Length of stay in days & $9.6(11.2)$ & $12.5(14.2)$ \\
\hline Median & 6 & 8 \\
\hline Surgical admission & $1531(40.3)$ & $1677(57.1)$ \\
\hline APACHE IV score ${ }^{\dagger}$ & $77.4(29.2)$ & NA (NA) \\
\hline \multicolumn{3}{|l|}{ Colonization status ${ }^{\dagger}$} \\
\hline - S. aureus positive & $390(10.3)$ & $216(7.4)$ \\
\hline - S. aureus negative & $2650(69.7)$ & $1487(50.1)$ \\
\hline - Unknown / missing & $761(20.2)$ & $1232(42.0)$ \\
\hline ICU mortality & $649(17.1)$ & $626(21.3)$ \\
\hline $\begin{array}{l}\text { Ventilator associated } \\
\text { pneumonia }\end{array}$ & $352(9.3)$ & $410(14.0)$ \\
\hline S. aureus & $41(1.1)$ & $42(1.43)$ \\
\hline Other pathogen & $263(6.9)$ & $245(8.4)$ \\
\hline Unknown / missing pathogen & $48(1.3)$ & $123(4.2)$ \\
\hline
\end{tabular}

+ measured at ICU admission

$\mathrm{SD}=$ standard deviation, $\mathrm{APACHE}=$ Acute Physiology \& Chronic Health Evaluation, ICU=Intensive Care Unit 


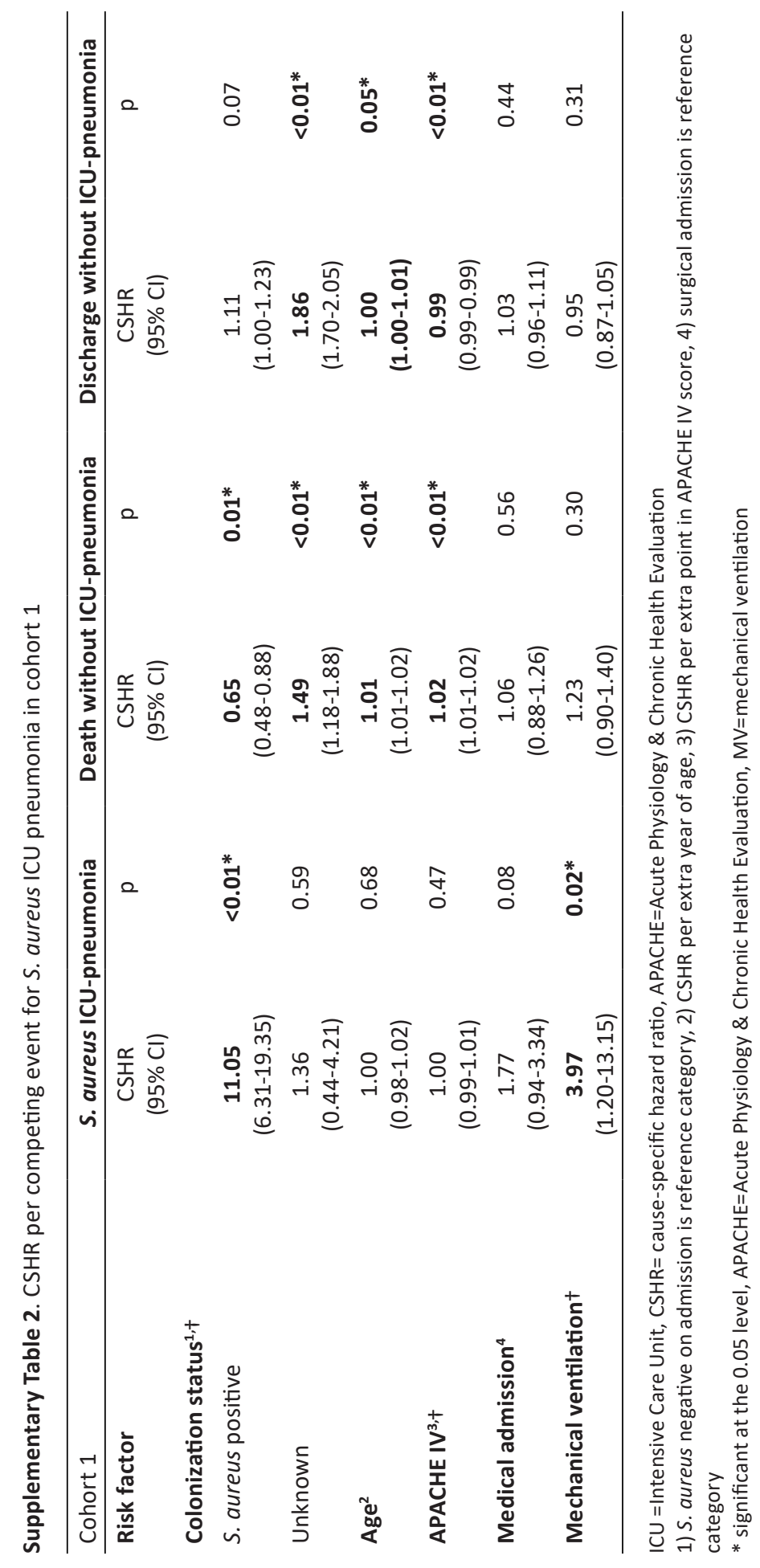




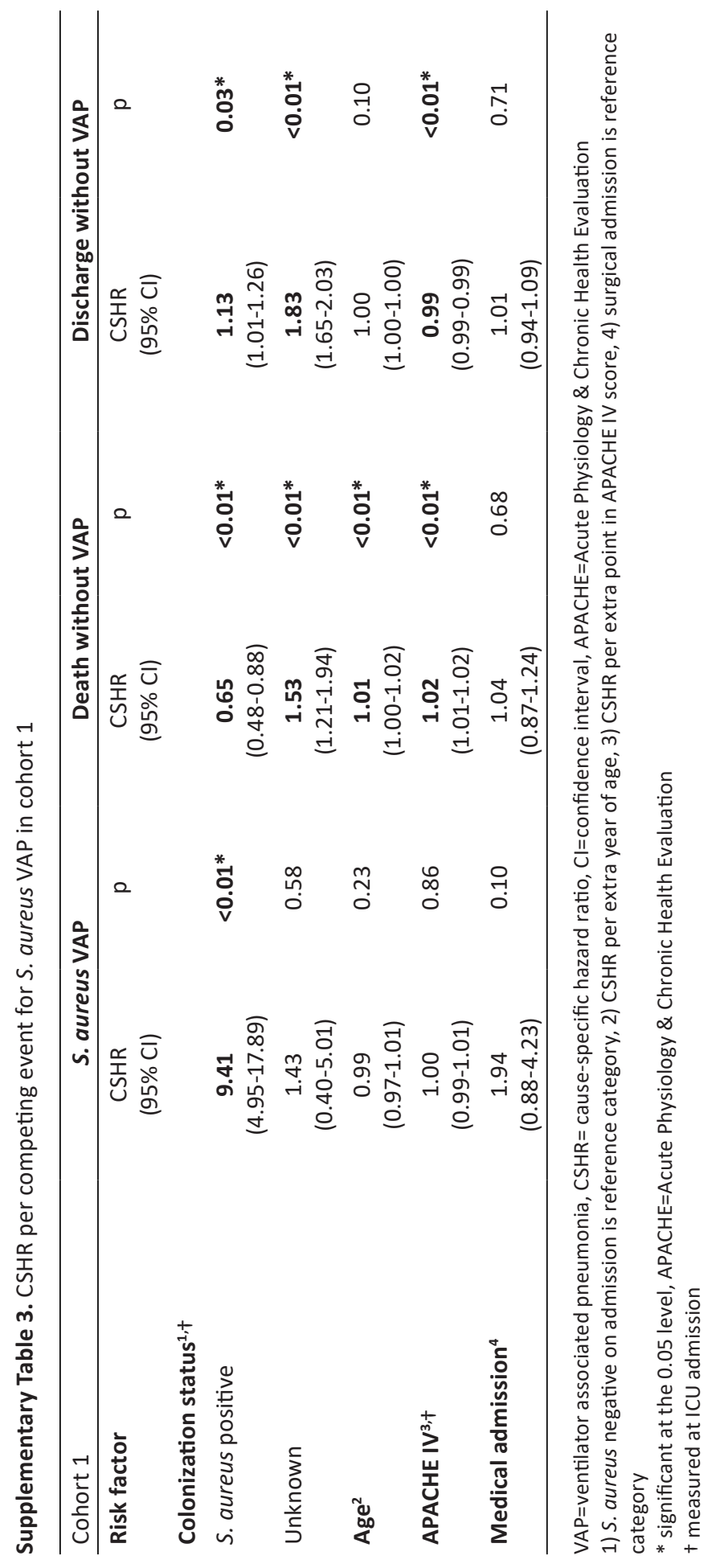




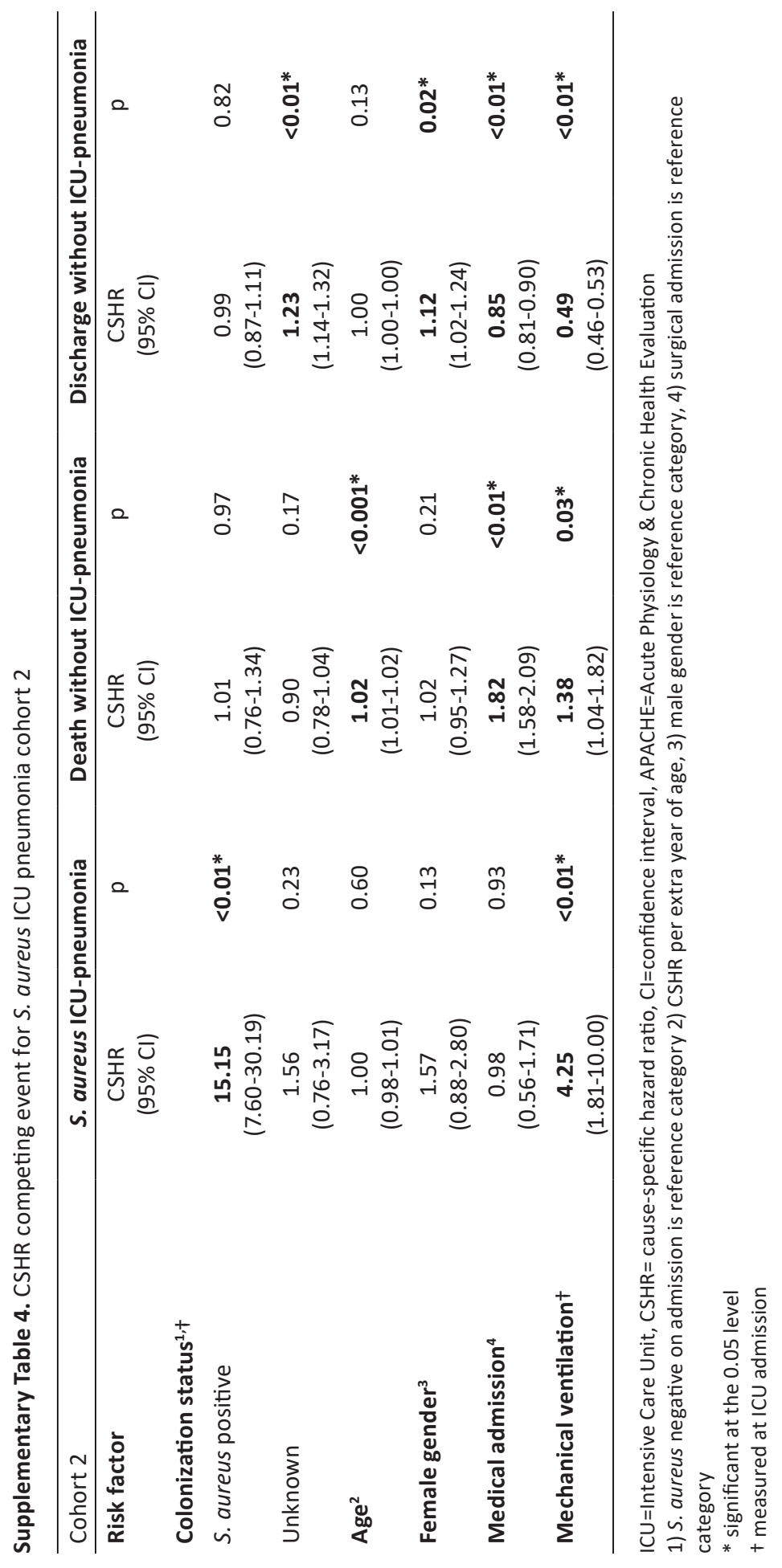




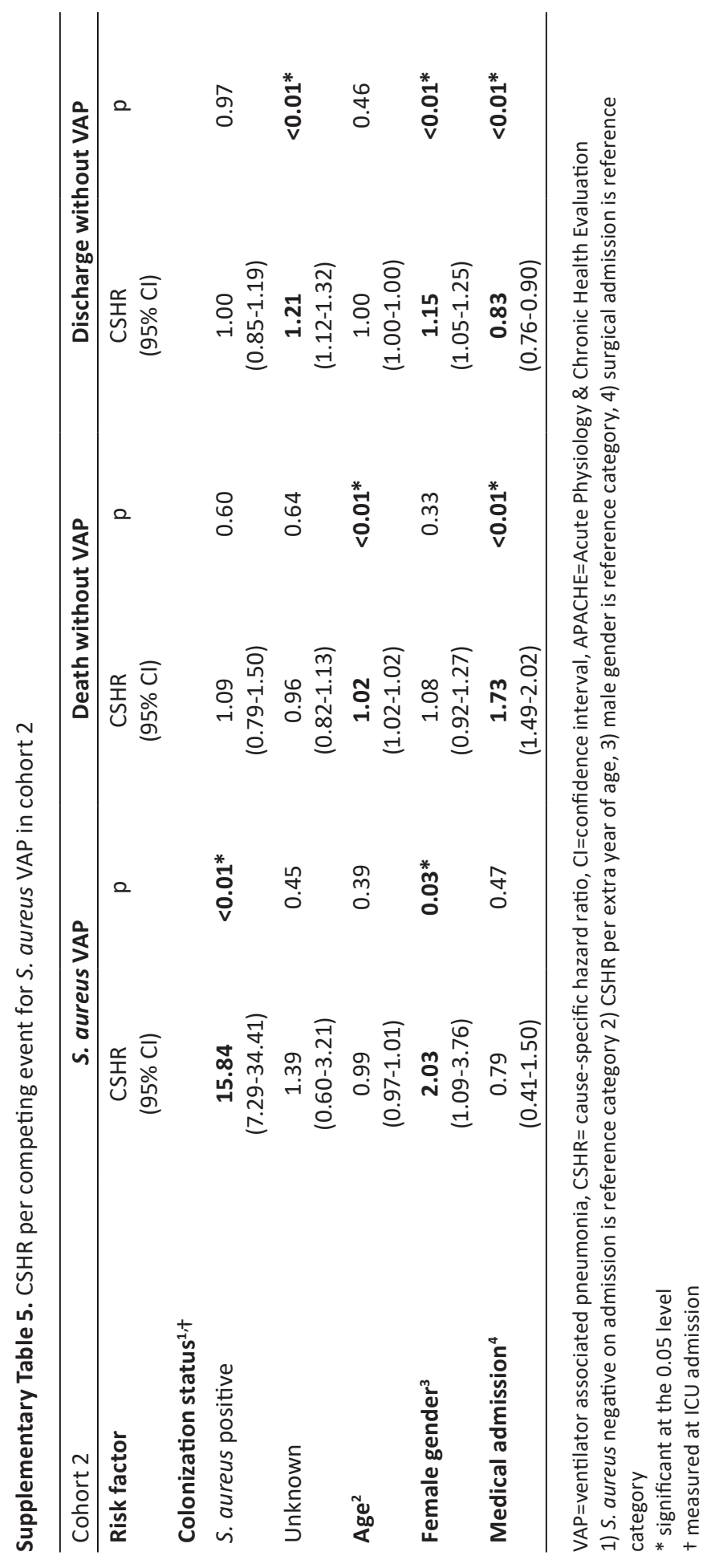


Supplementary Table 6. Incidence proportions of ICU pneumonia in patients colonized with $S$. aureus at ICU admission

\begin{tabular}{lcc}
\hline & Cohort $\mathbf{1}(\mathbf{n}=399 *)$ & Cohort $\mathbf{2}(\mathbf{n = 3 1 4 * )}$ \\
& $N(\%)$ & $N(\%)$ \\
\hline ICU pneumonia & $60(15.04)$ & $62(19.75)$ \\
S. aureus & $29(7.27)$ & $26(8.28)$ \\
Other pathogen & $24(6.02)$ & $19(6.05)$ \\
Unknown / missing pathogen & $7(1.75)$ & $17(5.41)$ \\
\hline
\end{tabular}

* Please note that for this analysis a subgroup of patients was used from both cohorts: only patients who were colonized with $S$. aureus at ICU admission were included. 
Supplementary Table 7. Incidence proportions of ventilator-associated pneumonia in patients colonized with S. aureus at ICU admission

\begin{tabular}{lcc}
\hline & Cohort $\mathbf{1}(\mathbf{n}=\mathbf{3 8 9 *})$ & Cohort $\mathbf{2}(\mathbf{n}=\mathbf{2 1 6 *})$ \\
& $N(\%)$ & $N(\%)$ \\
\hline Ventilator-associated pneumonia & $\mathbf{4 8 ( 1 2 . 3 3 )}$ & $\mathbf{4 7 ( 2 1 . 7 6 )}$ \\
S. aureus & $21(5.40)$ & $21(9.72)$ \\
Other pathogen & $21(5.40)$ & $18(8.33)$ \\
Unknown / missing pathogen & $6(1.54)$ & $8(3.70)$ \\
\hline
\end{tabular}

* Please note that for this analysis a subgroup of patients was used from both cohorts: only patients who were colonized with S. aureus at ICU admission AND ever on mechanical ventilation were included. 
Supplementary Table 8. Cumulative incidences (absolute and \%) of primary and secondary outcome, grouped by $S$. aureus colonization status at ICU admission.

\begin{tabular}{lccc}
\hline & $\begin{array}{c}\text { S. aureus positive } \\
\mathrm{N}(\%)\end{array}$ & $\begin{array}{c}\text { S. aureus } \\
\text { negative } \\
\mathrm{N}(\%)\end{array}$ & $\begin{array}{c}\text { S. aureus } \\
\text { unknown N (\%) }\end{array}$ \\
\hline Cohort 1: S. aureus ICU pneumonia & $29(7.27)$ & $21(0.76)$ & $4(0.44)$ \\
Cohort 1: S. aureus VAP & $21(5.38)$ & $17(0.64)$ & $3(0.39)$ \\
Cohort 2: S. aureus ICU pneumonia & $26(8.28)$ & $13(0.61)$ & $16(0.60)$ \\
Cohort 2: S. aureus VAP & $21(9.72)$ & $10(0.67)$ & $11(0.89)$ \\
\hline
\end{tabular}

VAP=ventilator associated pneumonia 


$$
\pi \pi
$$




\title{
Risk prediction for Staphylococcus aureus surgical site infection following cardiothoracic surgery; a secondary
analysis of the V710-P003 trial cardiothoracic surgery; a secondary
analysis of the V710-P003 trial
}

\section{CHAPTER}

\author{
Fleur P. Paling ${ }^{1}$ \\ Karina Olsen ${ }^{1}$ \\ Kristin Ohneberg ${ }^{2}$ \\ Martin Wolkewitz ${ }^{2}$ \\ Vance G. Fowler Jr. ${ }^{3}$ \\ Mark J. DiNubile ${ }^{4}$ \\ Hasan S. Jafri ${ }^{5}$ \\ Frangiscos Sifakis ${ }^{6}$ \\ Marc J.M. Bonten ${ }^{1,7}$ \\ Stephan J. Harbarth ${ }^{8}$ \\ Jan A.J.W. Kluytmans ${ }^{1,9}$
}

Published in: PLoS One. 2018 Mar 21;13(3):e0193445.

doi: 10.1371/journal.pone.0193445.

\footnotetext{
${ }^{1}$ Julius Center for Health Sciences and Primary Care, University Medical Center Utrecht, Utrecht, Netherlands

${ }^{2}$ Institute for Medical Biometry and Statistics, University Medical Center Freiburg, Freiburg, Germany

${ }^{3}$ Division of Infectious Diseases, Duke University Medical Center, Durham, North Carolina, United States of America

${ }^{4}$ Merck \& Company Incorporation, Kenilworth, New Jersey, United States of America

${ }^{5}$ Medlmmune, Gaithersburg, Maryland, United States of America

${ }^{6}$ AstraZeneca Pharmaceuticals LP, Gaithersburg, Maryland, United States of America

${ }^{7}$ Department of Medical Microbiology, University Medical Center Utrecht, Utrecht, Netherlands

${ }^{8}$ Geneva University Hospitals and Medical School, Geneva, Switzerland

${ }^{9}$ Amphia Hospital, Breda, The Netherlands
} 


\section{ABSTRACT}

Background: Identifying patients undergoing cardiothoracic surgery at high risk of Staphylococcus aureus surgical site infection (SSI) is a prerequisite for implementing effective preventive interventions. The objective of this study was to develop a risk prediction model for S. aureus SSI or bacteremia after cardiothoracic surgery based on pre-operative variables.

Materials / methods: Data from the Merck Phase IIb/III S. aureus vaccine (V710-P003) clinical trial were analyzed. In this randomized placebo-controlled trial, the effect of preoperative vaccination against $S$. aureus was investigated in patients undergoing cardiothoracic surgery. The primary outcome was deep/superficial S. aureus SSI or S. aureus bacteremia through day 90 after surgery. Performance, calibration, and discrimination of the final model were assessed.

Results: Overall 164 out of 7,647 included patients (2.1\%) developed S. aureus infection (149 SSI, 15 bacteremia, 28 both). Independent risk factors for developing the primary outcome were pre-operative colonization with S. aureus (OR 3.08, 95\% confidence interval [CI] 2.23-4.22), diabetes mellitus (OR 1.87, 95\% Cl 1.34-2.60), BMI (OR 1.02 per $\mathrm{kg} / \mathrm{m}^{2}, 95 \% \mathrm{Cl} 0.99-1.05$ ), and CABG (OR 2.67, 95\% Cl 1.91-3.78). Although vaccination had a significant (albeit modest) protective effect, it was omitted from the model because its addition did not significantly change the coefficients of the final model and V710-vaccine development has been discontinued due to insufficient efficacy. The final prediction model had moderate discriminative accuracy (AUC-value, 0.72).

Conclusion: Pre-operative S. aureus colonization status, diabetes mellitus, BMI, and type of surgical procedure moderately predicted the risk of S. aureus SSI and/or bacteremia among patients undergoing cardiothoracic surgery. 


\section{INTRODUCTION}

Surgical site infection (SSI) with or without bacteremia is a common post-operative complication responsible for increased morbidity, mortality, and health care costs[13]. The most important cause of SSIs among patients undergoing clean surgery is Staphylococcus aureus [4-6] which frequently colonizes the nares and skin in the healthy population. In preoperative patients, carriage is associated with an elevated risk for post-operative SSI and bacteremia [7,8]. Yet the ability to identify preoperative patients at highest risk for S. aureus SSI or post-operative bacteremia is inadequate [9]. As preemptive pathogen-specific preventive interventions are under development, it is important to reliably identify those patients at substantial risk for this complication [10].

For this study, data from the Merck Phase IIb/III S. aureus vaccine study (V710P003) were analyzed [11]. This double-blinded, randomized, placebo-controlled trial investigated the effect of a pre-operative vaccine targeting $S$. aureus on the incidence of postoperative S. aureus bacteremia and/or deep sternal wound infection in adult patients undergoing cardiothoracic surgery through postoperative day 90 . V710 was not sufficiently efficacious in preventing the primary endpoint by prespecified criteria, and overall mortality rates for the placebo or vaccine group were not significantly different. The trial was stopped prematurely after interim analysis showed lack of efficacy as well as a numerically higher mortality rate in the subset of vaccine recipients developing $S$. aureus infections. Pre-operative S. aureus colonization status was documented as part of protocol-stipulated procedures.

In the current post hoc analysis of the prospectively collected data from this clinical trial, we aimed to develop a pathogen-specific risk prediction model for S. aureus SSI and/or bacteremia in patients after cardiothoracic surgery based on information ascertainable preoperatively.

\section{Materials and methods}

Data from the randomized, double-blind, placebo-controlled trial of Merck Phase IIb/ III S. aureus vaccine (V710-P003, registered at clinicaltrials.gov under the identifier NCT00518687) were used for this post hoc analysis [11]. Because the clinical trial was stopped in part due to unacceptably low vaccine efficacy, we included both placebo and vaccine recipients in this analysis. Data were available on all efficacy outcomes. Decolonization procedures and pre-operative surgical prophylaxis were provided according to local standards of care for the international sites participating in the trial. However, decolonization methods were neither mandated by protocol nor routinely 
recorded. The original study protocol was approved by the institutional review boards or ethical review committees at each site and executed in accordance with Good Clinical Practice guidelines.

\section{Patient population}

Adult patients undergoing elective cardiothoracic surgery were eligible for inclusion. Exclusion criteria, described in more detail elsewhere, included active infection, pregnancy, and immunosuppression[11,12].

\section{Primary outcome}

The primary outcome was a binary (yes/no) composite endpoint through day 90 after surgery, which included at least one of the following S. aureus diagnoses: deep/ superficial sternal wound infection (including mediastinitis), deep/superficial harvest site infection, and bacteremia (defined as at least one positive blood culture growing S. aureus). All cases were adjudicated by an independent committee using diagnostic criteria established by the Center for Disease Control and Prevention (CDC) [13].

\section{Potential predictors and their management}

A list of candidate predictors was defined prior to initiating this analysis, based on clinical judgment and availability in the database, including pre-operative $S$. aureus colonization status, pre-operative antibiotic use, diabetes mellitus, type of cardiothoracic procedure, body mass index (BMI), age, and sex.

We defined a patient to be colonized if nasal S. aureus carriage was documented by culture at any moment before surgery. This assumption was chosen because literature indicates that colonization status is largely dependent on the patient's constitution and thus relatively constant over time[7].

Pre-operative antibiotic use was defined as any systemic antibiotic use within 6 months before surgery, excluding pre-operative prophylaxis. A timeframe of 6 months pre-operatively was chosen, considering that previous studies had shown that the microbiome can be affected after antibiotic usage for this period of time[14]. Diabetes mellitus was coded as yes if there was a confirmed diagnosis of diabetes mellitus, regardless of duration of disease or need for diabetic agents. Gestational diabetes was not included. Surgical procedure type was dichotomized to coronary artery bypass grafting (CABG) or not. The combination of CABG and cardiac valve surgery was coded as $C A B G$. Cardiac valve surgery alone or other cardiothoracic surgery types including median sternotomy were coded as 'no CABG'. 
Age and BMI were used as continuous variables; it was checked whether fractional polynomials improved model performance[15]. Missing values $(n=152)$ of $S$. aureus colonization status were imputed using multiple imputation techniques[16].

Univariate logistic regression analysis was performed on the mentioned variables. Variables with a univariate $p \leq 0.157$ were entered into the final multivariable model, roughly corresponding to the selection threshold based on the Akaike information criterion when considering $p$-values [17]. Tests of interactions between pre-operative $S$. aureus colonization status and BMI or diabetes mellitus were performed ( $p$-value $<0.05$ ).

\section{Regression model and model performance}

A logistic regression model was fitted with the variables described above. Overall model performance was assessed by measuring the explained variation (Nagelkerke $R^{2}$ )[18]. Calibration of the model was assessed by plotting the observed proportion of events against the predicted risks for groups defined by ranges of individual predicted risks. For the assessment of the discrimination of the model, a receiver operating characteristic (ROC) curve was plotted and the area under the curve (AUC or c-statistic) was computed. Internal validation was assessed by performing 200 bootstrap samples.

\section{Sensitivity analyses}

\section{Competing events}

Patients might have died within 90 days post-surgery without reaching the primary outcome, which means that death is a competing event for the primary outcome. As a sensitivity analysis, a Fine \& Gray model was fitted to account for the time-to-event, considering death as a competing event [19]. Subdistribution hazard ratios for SSI were calculated as an alternative measure (by acknowledging the time-dependency) for the odds ratios. Cumulative incidence functions were calculated with stratification by risk score groups using the Aalen-Johansen estimator[20].

\section{Vaccine effect}

Considering that we used a slightly different primary outcome compared to the initial study (originally superficial or harvest site infections were not included), it was assessed whether a vaccine-effect was present $(p$-value $<0.05)$ and whether adding vaccination to the model significantly altered the effect estimates.

All statistical analyses were performed using R version 2.10.00. [21] 


\section{RESULTS}

In the final analysis, 7,647 patients were included. Their baseline characteristics are described in table 1. Overall 165 out of 7,647 included patients (2.1\%) developed $S$. aureus SSI and/or bloodstream infection, including 122 (1.6\%) patients with SSI without bacteremia, $28(0.4 \%)$ patients with bacteremic SSI, and 15 patients $(0.2 \%)$ with postoperative bacteremia without SSI.

\section{Predictors of $S$. aureus SSI and/or bacteremia}

Several pre-operative variables were univariately associated with the primary outcome: pre-operative colonization status with S. aureus (OR 3.07, 95\% confidence interval [CI] 2.23-4.20), diabetes mellitus (OR 2.45, 95\% Cl 1.78-3.34), CABG (OR 3.01, 95\% Cl 2.244.35), and $\mathrm{BMI}$ (OR 1.04 per $\mathrm{kg} / \mathrm{m}^{2}$ increase, $95 \% \mathrm{Cl} 1.02-1.07$ ). No significant interaction was found between pre-operative $S$. aureus colonization and either BMI or diabetes mellitus ( $p$-values 0.196 and 0.089 , respectively).

Independent risk factors identified during multivariate analysis were pre-operative colonization status (OR 3.08, 95\% Cl 2.23-4.22), diabetes mellitus (OR 1.87, 95\% Cl 1.34-2.60), CABG (OR 2.67, 95\% CI 1.91-3.78) and BMI (OR 1.02 per unit increase, $95 \%$ Cl 0.99-1.05) (Table 2).

Table 1. Baseline characteristics

\begin{tabular}{|c|c|c|c|}
\hline & $\begin{array}{l}\text { With outcome } \\
\mathrm{N}=165\end{array}$ & $\begin{array}{l}\text { Without outcome } \\
N=7,482\end{array}$ & $\begin{array}{l}\text { Total } \\
\mathrm{N}=7,647\end{array}$ \\
\hline Age (years) & $64.9(10.8)$ & $63.9(12.4)$ & $63.9(12.4)$ \\
\hline Gender: female & $53(30.0)$ & $2,467(33.0)$ & $2,520(33.0)$ \\
\hline $\begin{array}{l}\text { Pre-operative } S \text {. aureus } \\
\text { colonization }\end{array}$ & $67(42.0)$ & $1,364(18.2)$ & 1,431 (18.7) \\
\hline BMI $\left(\mathrm{kg} / \mathrm{M}^{2}\right)$ & $29.0(5.7)$ & $27.6(5.3)$ & $27.6(5.3)$ \\
\hline Diabetes mellitus & $71(45.3)$ & $1,765(23.6)$ & $1836(24.0)$ \\
\hline Pre-operative antibiotic use & $10(5.3)$ & $653(8.7)$ & $663(8.7)$ \\
\hline CABG & $113(68.5)$ & $3,075(41.1)$ & $3,188(41.7)$ \\
\hline Vaccination & $66(40.1)$ & $3,747(50.0)$ & $3,813(49.9)$ \\
\hline Death* & $7(4.2)$ & $229(3.1)$ & 236 (3.1) \\
\hline
\end{tabular}

Values are given as means (SD), and numbers (\%).

$\mathrm{SD}=$ standard deviation, $\mathrm{BMI}=$ body mass index, $\mathrm{CABG}=$ coronary artery bypass grafting

*Death within 90 days post-surgery 


\section{Model performance}

The mean explained variation of the model as indicated by the Nagelkerke $R^{2}$ was 0.08 . The distribution of predicted risks for the event of interest was highly skewed to the left, with more patients in the low risk categories than in the high-risk categories. Only $8.2 \%$ of the patients had a risk of $\geq 5 \%$. Of the 209 S. aureus colonized, diabetic patients undergoing $\mathrm{CABG}$ (i.e. who had all three major risk factors), the risk of developing the event was $11 \%$ ( $n=23$ ). Of the 3,012 patients without any preoperative risk factor, 28 (0.9\%) developed the event.

Table 2. Univariate and multivariate logistic regression analysis

\begin{tabular}{lllll}
\hline & $\begin{array}{l}\text { Unadjusted OR } \\
(\mathbf{9 5 \%} \mathrm{Cl})\end{array}$ & p-value & $\begin{array}{l}\text { Adjusted OR } \\
(\mathbf{9 5 \%} \mathrm{Cl})\end{array}$ & p-value \\
\hline Age $^{1}$ & $1.01(0.99-1.02)$ & 0.315 & Not included & \\
Gender: female $^{2}$ & $0.96(0.69-1.33)$ & 0.818 & Not included & \\
$\begin{array}{l}\text { Pre-operative S.aureus } \\
\text { colonization }\end{array}$ & $3.01(2.23-4.20)$ & $<0.001^{*}$ & $3.08(2.23-4.22)$ & $<0.001^{*}$ \\
BMI $^{1}$ & $1.04(1.02-1.07)$ & $\mathbf{0 . 0 0 1 *}$ & $1.02(0.99-1.05)$ & 0.148 \\
Diabetes mellitus & $2.45(1.78-3.34)$ & $<0.001^{*}$ & $1.87(1.34-2.60)$ & $<0.001^{*}$ \\
Pre-operative antibiotic & $0.67(0.33-1.22)$ & 0.231 & Not included & \\
use & $3.10(2.24-4.35)$ & $<0.001^{*}$ & $2.67(1.91-3.78)$ & $<0.001^{*}$ \\
CABG & $0.67(0.48-0.91)$ & $0.011^{*}$ & $0.67(0.48-0.91)$ & $0.012^{*}$ \\
Vaccination & & & & \\
\hline
\end{tabular}

* Significant at the 0.05 level. OR=odds ratio

1) OR per year of age or $\mathrm{kg} / \mathrm{M}^{2}$ increase, 2) Male is reference category

Figure 1 shows a calibration plot with average agreement between the observed events and the predicted risks by ranges of individual predicted risks (Hosmer-Lemeshow $\chi 2=13.0, p=0.11$ ). Discrimination of the model was average, with an area under the ROC curve of 0.72 (95\% Cl 0.68-0.76) (Figures 2 and 3).

\section{Internal validation}

The stability of the final model was further assessed in 200 bootstrap samples. Using these samples, we derived an $\mathrm{R}^{2}$ of 0.07 and AUC of 0.72 after correction for optimism. The Somers' Dxy rank correlation between predicted probabilities and observed responses was 0.43 ( 0 indicating completely random predictions and 1 indicating perfect predictions). 


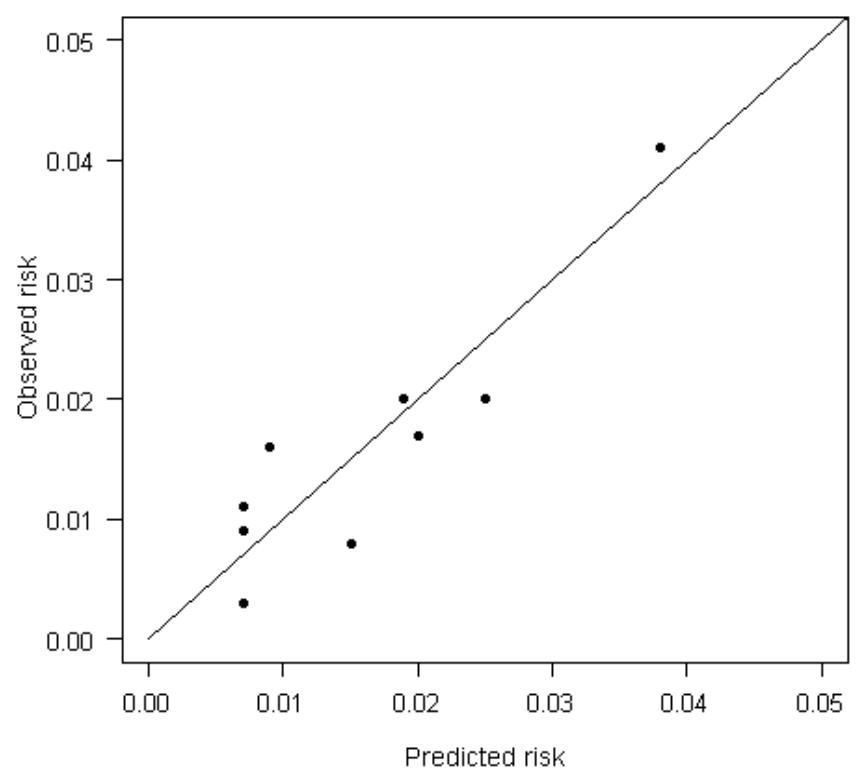

Figure 1. Calibration plot of final model, showing observed risks vs. predicted risks on the primary outcome.

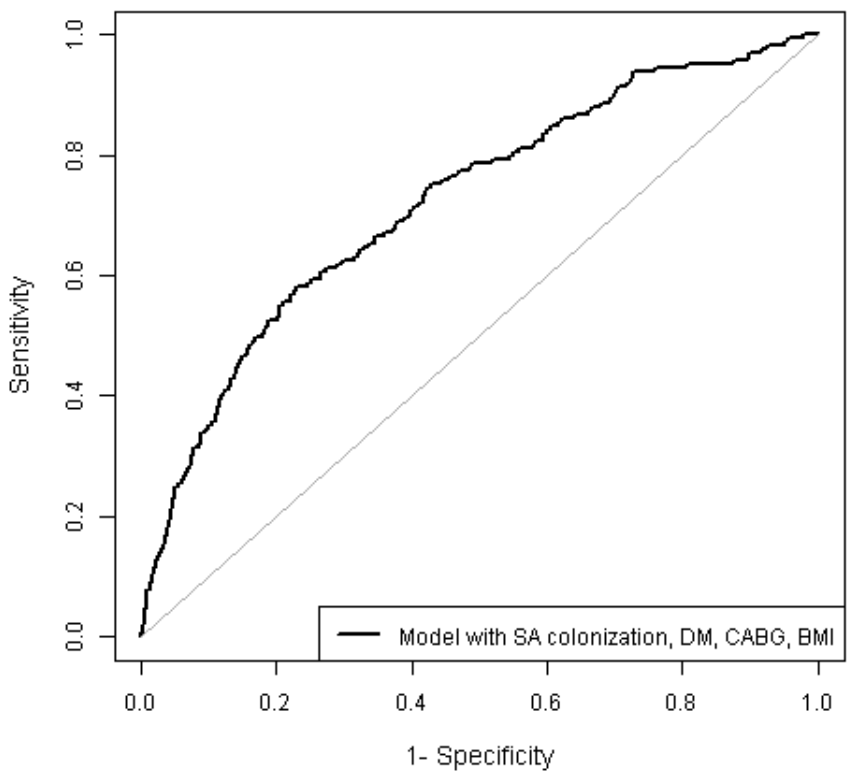

Figure 2. ROC curve of final model, with an AUC of 0.72 ( $95 \% \mathrm{Cl} 0.68-0.76)$. 


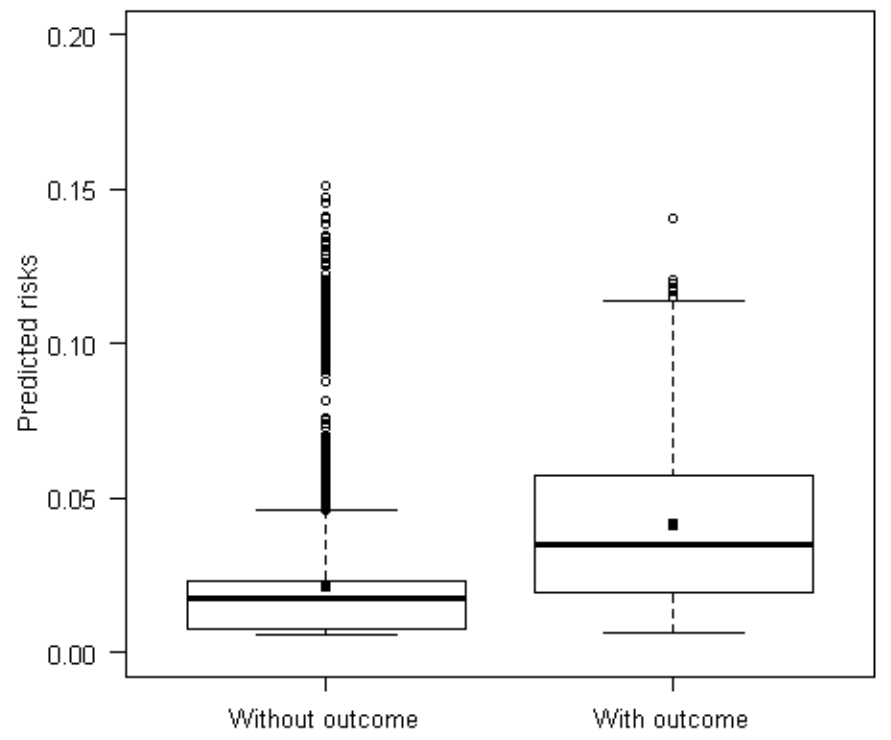

Figure 3. Boxplot showing distribution of predicted risks stratified for groups with/without primary outcome.

\section{Sensitivity analysis}

\section{Competing risks}

A total number of 236 patients died within 90 days post-surgery. Of these, 229 had not yet developed the primary event of interest. Using the Fine \& Gray competing risks analysis to assess whether the subdistribution hazard ratios differ from the odds ratios from the logistic regression model, the estimates did not change significantly (maximum observed change was $2 \%$ ). Hence, the effect of death as a competing risk can largely be ignored.

\section{Vaccine effect}

Vaccination was univariately associated with the primary outcome. V710 was protective against $S$. aureus infection (OR $0.67,95 \% \mathrm{Cl} 0.48-0.91, \mathrm{p}=0.011$ ), and remained so after correction for other predictors (OR $0.67,95 \% \mathrm{Cl} 0.48-0.91, p=0.012$ ). However, other predictor estimates did not change significantly after incorporating vaccination status, indicating a lack of confounding effect. Furthermore, because the development of this specific vaccine has been discontinued, vaccination was not included as a predictor in the final model. 


\section{DISCUSSION}

In this analysis, we built a risk prediction model to determine which preoperative characteristics put patients at higher risk of developing S. aureus SSI and/or bacteremia after cardiothoracic surgery. We identified $S$. aureus colonization, diabetes, increasing $\mathrm{BMI}$, and CABG surgery as independent risk factors. The final prediction model using these readily available predictors performed satisfactorily.

As the frequency and impact of post-surgical infections remain substantial, the relevance of an accurate prediction model remains. Many previous studies have developed and validated risk prediction tools for all-cause surgical site infection in cardiothoracic patients, some of which are frequently used in practice [22,23]. However, practical pathogen-specific models for postoperative $S$. aureus infections are scarce. Pathogen-specific prediction may be preferable, anticipating the arrival of targeted preventive measures in the near future [10,24-26]. Furthermore, patients suffering from S. aureus infections are at substantial risk for bad outcomes and incur higher health care costs[27-30]. This prediction model advances existing literature because it employs simple predictors routinely available in the preoperative patient. The risk difference between a patient not having any risk factor compared with one that has three is $10.1 \%$ (0.9\% vs. $11.0 \%)$. However, in this derivation set, even though the predictors frequently occurred independently of each other, there were only 209 patients (2.7\%) having all three factors, still leaving many patients at low or intermediate risk. A previous study by Kanafani et al. showed similar results [9]. Better discrimination between infected and non-infected patients is required to identify a larger patient group that would benefit from new interventions. Comprehensive prospective studies will be required, such as the prospective cohort study called ASPIRE-SSI (Advanced Understanding of Staphylococcus Aureus Infections in Europe - Surgical Site Infections), which is part of the COMBACTE-NET initiative[31,32]. This study will describe risk factors for $S$. aureus SSI of approximately 5000 patients across Europe undergoing different types of surgery and is currently ongoing.

A possible option for new model developers could be to use an established, validated prediction score like Euroscore and assess whether adding pathogen-specific variables like colonization status can make the model pathogen-specific[33]. This could have wider implications, considering that implementation would not require any major change in routine practice, should the new prediction model be successful. The recently published 'Global guidelines on the prevention of surgical site infection' specifically stress the need for such a simple, inexpensive screening process, considering that in 
low- and middle-income countries the logistical and financial burdens that come with a screening and decolonization intervention may be too burdensome to implement on all preoperative patients [34].

A major strength of the current study is the size of the study and the number of participating countries/centers. Furthermore, data collection and patient follow-up was stipulated by protocol and closely monitored, minimizing the amount of missing data during follow-up, and ensuring a high proportion of patients screened for $S$. aureus colonization unlikely to occur outside the setting of a clinical trial. Last, but not least, the statistical analyses performed here, including the sensitivity analyses taking into account competing risks were sophisticated and comprehensive.

There are several limitations to this analysis. First of all, decolonization strategies for S. aureus were neither standardized nor documented. Decolonization methods were likely applied to colonized patients at a majority of the sites [35]. If indeed accurate, this practice would decrease the difference in incidence rate of the primary outcome between colonized and non-colonized patients, as decolonization reduces infection rates in carriers [36,37].

Furthermore, in this study only nares were screened for $S$. aureus colonization, thus, carriage on skin or at other sites may have been missed. In other words, there is potential misclassification bias, since some of the "non-colonized" patients may have been colonized elsewhere. This misclassification likely would be independent of $S$. aureus bacteremia and SSI, giving rise to a non-differential misclassification of the S. aureus carrier status. The non-differential misclassification may have biased our estimates towards the null and reduced the discriminative effect of the new prediction model.

Despite the limitations described above, the model performed moderately well. In its present form it may only be useful to indicate an especially high risk for patients having all three risk factors. For subtler prediction and external validation, further enhancement of the model is necessary. 


\section{CONCLUSION}

From this analysis, we can conclude that pre-operative $S$. aureus colonization gives a $3 x$ higher OR for S. aureus SSI / bacteremia in the unsubstantiated (but likely) presence of decolonization procedures. Without decolonization, the risk is likely to be higher. This model that included colonization status, diabetes, and CABG had overall average performance.

Acknowledgments: The authors would like to thank Dr. Flaminia Olearo (Geneva) for her contributions to the project.

Funding: This research project has received support from the Innovative Medicines Initiative Joint Undertaking under grant agreement no. 115523 resources which are composed of financial contribution from the European Union Seventh Framework Programme (FP7/2007-2013) and EFPIA companies in kind contribution. This project used data collected earlier in a randomized-controlled study sponsored and funded by Merck \& Co. Inc., Kenilworth, NJ, USA. 


\section{REFERENCES}

1. Douglas Scott II R, Promotion. NC for PD and C of ID (Centers for DC and PD of HQ. The Direct medical costs of healthcare-associated infections in U.S. hospitals and the benefits of prevention [Internet]. 2009. Available: https://www.cdc.gov/HAl/pdfs/hai/Scott_CostPaper. pdf

2. Kirkland KB, Briggs JP, Trivette SL, Wilkinson WE, Sexton DJ. The impact of surgical-site infections in the 1990s: attributable mortality, excess length of hospitalization, and extra costs. Infect Control Hosp Epidemiol. 1999;20: 725-30. doi:10.1086/501572

3. Gottlieb GS, Fowler VG, Kong LK, McClelland RS, Gopal AK, Marr KA, et al. Staphylococcus aureus bacteremia in the surgical patient: a prospective analysis of 73 postoperative patients who developed Staphylococcus aureus bacteremia at a tertiary care facility. J Am Coll Surg. 2000;190: 50-7. Available: http://www.ncbi.nlm.nih.gov/pubmed/10625232

4. Young B, Ng TM, Teng C, Ang B, Tai HY, Lye DC. Nonconcordance with surgical site infection prevention guidelines and rates of surgical site infections for general surgical, neurological, and orthopedic procedures. Antimicrob Agents Chemother. 2011;55: 4659-63. doi:10.1128/ AAC. 00562-11

5. Anderson DJ. Surgical site infections. Infect Dis Clin North Am. 2011;25: 135-53. doi:10.1016/j. idc.2010.11.004

6. Anderson DJ, Kaye KS. Staphylococcal surgical site infections. Infect Dis Clin North Am. 2009;23: 53-72. doi:10.1016/j.idc.2008.10.004

7. Wertheim HFL, Melles DC, Vos MC, van Leeuwen W, van Belkum A, Verbrugh HA, et al. The role of nasal carriage in Staphylococcus aureus infections. Lancet Infect Dis. 2005;5: 751-62. doi:10.1016/S1473-3099(05)70295-4

8. Gervaz P, Bandiera-Clerc C, Buchs NC, Eisenring M-C, Troillet N, Perneger T, et al. Scoring system to predict the risk of surgical-site infection after colorectal resection. Br J Surg. 2012;99: 589-595. doi:10.1002/bjs.8656

9. Kanafani ZA, Arduino JM, Muhlbaier LH, Kaye KS, Allen KB, Carmeli Y, et al. Incidence of and preoperative risk factors for Staphylococcus aureus bacteremia and chest wound infection after cardiac surgery. Infect Control Hosp Epidemiol. 2009;30: 242-8. doi:10.1086/596015

10. Kostyanev T, Bonten MJM, O'Brien S, Steel H, Ross S, François B, et al. The Innovative Medicines Initiative's New Drugs for Bad Bugs programme: European public-private partnerships for the development of new strategies to tackle antibiotic resistance. J Antimicrob Chemother. Oxford University Press; 2016;71: 290-5. doi:10.1093/jac/dkv339 
11. Fowler VG, Allen KB, Moreira ED, Moustafa M, Isgro F, Boucher HW, et al. Effect of an investigational vaccine for preventing Staphylococcus aureus infections after cardiothoracic surgery: a randomized trial. JAMA. 2013;309: 1368-78. doi:10.1001/jama.2013.3010

12. Allen KB, Fowler VG, Gammie JS, Hartzel JS, Onorato MT, DiNubile MJ, et al. Staphylococcus aureus Infections After Elective Cardiothoracic Surgery: Observations From an International Randomized Placebo-Controlled Trial of an Investigational S aureus Vaccine. Open Forum Infect Dis. 2014;1: ofu071. doi:10.1093/ofid/ofu071

13. Centers for Disease Control. Surgical Site Infection ( SSI ) Event. 2014.

14. Malhotra-Kumar S, Lammens C, Coenen S, Van Herck K, Goossens H. Effect of azithromycin and clarithromycin therapy on pharyngeal carriage of macrolide-resistant streptococci in healthy volunteers: a randomised, double-blind, placebo-controlled study. Lancet (London, England). 2007;369: 482-90. doi:10.1016/S0140-6736(07)60235-9

15. Royston P, Sauerbrei W. Multivariable model-building: a pragmatic approach to regression anaylsis based on fractional polynomials for modelling continuous variables. John Wiley \& Sons;

16. Sterne JAC, White IR, Carlin JB, Spratt M, Royston P, Kenward MG, et al. Multiple imputation for missing data in epidemiological and clinical research: potential and pitfalls. BMJ. 2009;338: b2393. doi:10.1136/bmj.b2393

17. Sauerbrei W. The Use of Resampling Methods to Simplify Regression Models in Medical Statistics. J R Stat Soc Ser C (Applied Stat. Blackwell Publishers Ltd.; 1999;48: 313-329. doi:10.1111/1467-9876.00155

18. Mittlböck M, Schemper M. Explained variation for logistic regression. Stat Med. 1996;15: 1987-1997. doi:10.1002/(SICI)1097-0258(19961015)15:19<1987::AID-SIM318>3.0.CO;2-9

19. Fine JP, Gray RJ. A Proportional Hazards Model for the Subdistribution of a Competing Risk. J Am Stat Assoc. 1999;94: 496-509. doi:10.1080/01621459.1999.10474144

20. Allignol A, Schumacher M, Beyersmann J. A Note on Variance Estimation of the AalenJohansen Estimator of the Cumulative Incidence Function in Competing Risks, with a View towards Left-Truncated Data. Biometrical J. 2010;52: 126-137. doi:10.1002/bimj.200900039

21. R Core Team. R: A Language and Environment for Statistical Computing. R Foundation for Statistical Computing [Internet]. 2017. Available: https://www.r-project.org

22. Fowler VG, O'Brien SM, Muhlbaier LH, Corey GR, Ferguson TB, Peterson ED. Clinical predictors of major infections after cardiac surgery. Circulation. 2005;112: I358-65. doi:10.1161/ CIRCULATIONAHA.104.525790 
23. Nashef SAM, Roques F, Hammill BG, Peterson ED, Michel P, Grover FL, et al. Validation of European System for Cardiac Operative Risk Evaluation (EuroSCORE) in North American cardiac surgery. Eur J Cardiothorac Surg. 2002;22: 101-5. Available: http://www.ncbi.nlm. nih.gov/pubmed/12103381

24. François B, Jafri HS, Bonten M. Alternatives to antibiotics. Intensive Care Med. 2016;42: 2034-2036. doi:10.1007/s00134-016-4339-y

25. Giersing BK, Dastgheyb SS, Modjarrad K, Moorthy V. Status of vaccine research and development of vaccines for Staphylococcus aureus. Vaccine. 2016;34: 2962-2966. doi:10.1016/j.vaccine.2016.03.110

26. Frenck RW, Buddy Creech C, Sheldon EA, Seiden DJ, Kankam MK, Baber J, et al. Safety, tolerability, and immunogenicity of a 4-antigen Staphylococcus aureus vaccine (SA4Ag): Results from a first-in-human randomised, placebo-controlled phase 1/2 study. Vaccine. 2017;35: 375-384. doi:10.1016/j.vaccine.2016.11.010

27. Broex ECJ, van Asselt ADI, Bruggeman CA, van Tiel FH. Surgical site infections: how high are the costs? J Hosp Infect. 2009;72: 193-201. doi:10.1016/j.jhin.2009.03.020

28. McGarry SA, Engemann JJ, Schmader K, Sexton DJ, Kaye KS. Surgical-site infection due to Staphylococcus aureus among elderly patients: mortality, duration of hospitalization, and cost. Infect Control Hosp Epidemiol. 2004;25: 461-7. doi:10.1086/502422

29. Noskin GA, Rubin RJ, Schentag JJ, Kluytmans J, Hedblom EC, Smulders M, et al. The burden of Staphylococcus aureus infections on hospitals in the United States: an analysis of the 2000 and 2001 Nationwide Inpatient Sample Database. Arch Intern Med. American Medical Association; 2005;165: 1756-61. doi:10.1001/archinte.165.15.1756

30. Olsen MA, Krauss M, Agniel D, Schootman M, Gentry CN, Yan Y, et al. Mortality associated with bloodstream infection after coronary artery bypass surgery. Clin Infect Dis. 2008;46: 1537-46. doi:10.1086/587672

31. Rex JH. ND4BB: addressing the antimicrobial resistance crisis. Nat Rev Microbiol. Nature Publishing Group, a division of Macmillan Publishers Limited. All Rights Reserved.; 2014;12: 231-232. doi:10.1038/nrmicro3245

32. Combatting Bacterial Resistance in Europe [Internet]. Available: www.combacte.com

33. Roques F, Nashef SA, Michel P, Gauducheau E, de Vincentiis C, Baudet E, et al. Risk factors and outcome in European cardiac surgery: analysis of the EuroSCORE multinational database of 19030 patients. Eur J Cardiothorac Surg. 1999;15: 816-22-3. Available: http://www.ncbi. nlm.nih.gov/pubmed/10431864 
34. Allegranzi B, Zayed B, Bischoff P, Kubilay NZ, de Jonge S, de Vries F, et al. New WHO recommendations on intraoperative and postoperative measures for surgical site infection prevention: an evidence-based global perspective. Lancet Infect Dis. 2016;16: e288-e303. doi:10.1016/S1473-3099(16)30402-9

35. Noskin GA, Rubin RJ, Schentag JJ, Kluytmans J, Hedblom EC, Jacobson C, et al. Budget impact analysis of rapid screening for Staphylococcus aureus colonization among patients undergoing elective surgery in US hospitals . Infect Control Hosp Epidemiol. 2008;29: 16-24. doi:10.1086/524327

36. Bode LGM, Kluytmans JAJW, Wertheim HFL, Bogaers D, Vandenbroucke-Grauls CMJE, Roosendaal $\mathrm{R}$, et al. Preventing surgical-site infections in nasal carriers of Staphylococcus aureus. N Engl J Med. 2010;362: 9-17. doi:10.1056/NEJMoa0808939

37. van Rijen $M$, Bonten $M$, Wenzel R, Kluytmans J. Mupirocin ointment for preventing Staphylococcus aureus infections in nasal carriers. Cochrane database Syst Rev. 2008; CD006216. doi:10.1002/14651858.CD006216.pub2 


$$
\pi \pi
$$




\section{P. aeruginosa colonization at ICU admission as a risk factor for developing $P$. aeruginosa ICU pneumonia}

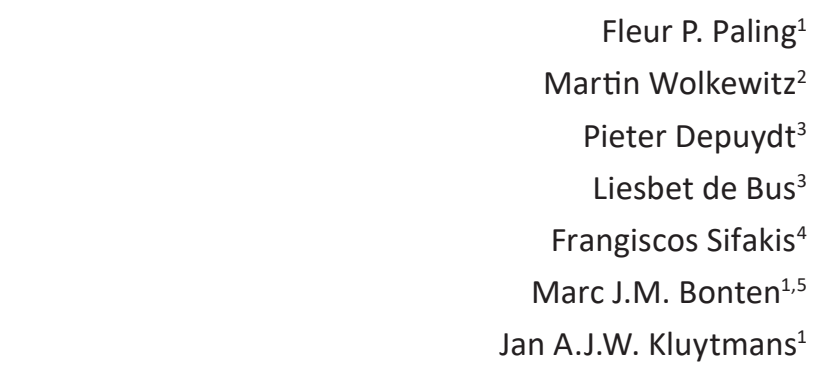

Published (as short report) in: Antimicrob Resist Infect Control. 2017 Apr 20;6:38. doi: 10.1186/s13756-017-0197-9.

\section{CHAPTER}

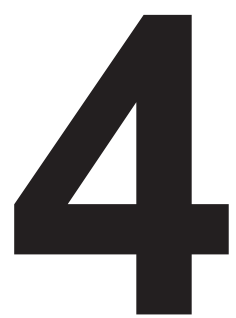

\footnotetext{
${ }^{1}$ Julius Center for Health Sciences and Primary Care, University Medical Center Utrecht, Utrecht, Netherlands

${ }^{2}$ Institute for Medical Biometry and Statistics, University Medical Center Freiburg, Freiburg, Germany

${ }^{3}$ Department of Intensive Care Medicine, University Hospital of Ghent, Ghent, Belgium

${ }^{4}$ AstraZeneca LP, Gaithersburg, Maryland, U.S.A.

${ }^{5}$ Department of Medical Microbiology, University Medical Center Utrecht, Utrecht, Netherlands
} 


\section{ABSTRACT}

Objective: To determine the incidence of $P$. aeruginosa (PA) ICU pneumonia and its independent association with PA colonization at ICU admission.

Methods: This was a post-hoc analysis of a prospectively collected cohort study. Adult ICU patients with a length of stay of $\geq 48 \mathrm{~h}$ were included and assessed for microbiologically confirmed PA ICU pneumonia. Multivariate survival analysis was performed, including the covariates age, gender, PA colonization at ICU admission, ICU admission specialty and mechanical ventilation at ICU admission, while taking into account the effect of competing risks.

Results: We included 5093 patients, 2447 (48\%) were tested for colonization; of those 226 (9.2\%) were PA colonized at ICU admission. The incidence of PA ICU pneumonia was $1.34 \%(n=68)$. PA colonization was an independent risk factor (subdistribution hazard ratio $[\mathrm{SHR}]$ 8.8; 95\% confidence interval $[\mathrm{Cl}]$ 4.9-15.7), as was mechanical ventilation (SHR 5.3, 95\% Cl 2.7-10.6).

Conclusion: In this study the incidence of $P$. aeruginosa ICU pneumonia was $1.34 \%$. Hazard ratios for PA colonized patients compared to non-colonized to develop PA ICU pneumonia were 8.8. The high risk associated with $P$. aeruginosa colonization for subsequent infection may offer a target for future interventions. 


\section{INTRODUCTION}

$P$. aeruginosa $(\mathrm{PA})$ is a frequently occurring nosocomial pathogen, causing potentially life threating infections, one of them being Intensive Care Unit (ICU) pneumonia, or pneumonia acquired while hospitalized on the ICU [1], [2]. PA colonization might be a risk factor for PA ICU pneumonia, but the bacterium may also be an innocent bystander in patients with pneumonia caused by another pathogen[1], [3], [4]. The association between PA carriage on ICU admission and the occurrence of PA ICU pneumonia remains relatively unexplored.

\section{OBJECTIVE}

To estimate the incidence of PA ICU pneumonia and its independent association with PA colonization at ICU admission.

\section{METHODS}

This analysis was performed on the data of a prospectively collected observational cohort study, performed in a mixed ICU of a tertiary hospital in Belgium. Data on epidemiology of ICU-acquired infections were collected from January 2010 until June 2014 , by means of the locally developed COSARA software application, allowing a continuous prospective registration of all infection- and antibiotic-related data.[5]

Adult patients with a length of stay of $\geq 48 \mathrm{~h}$ were included; screening for PA was part of routine care in patients with an expected length of stay of $\geq 48 \mathrm{~h}$ and was based on endotracheal aspirate (ETA), oropharyngeal and/or rectal cultures. Pneumonia diagnosis was based on radiologic criteria in combination with at least 1 clinical or laboratory criterion. Confirmed PA ICU pneumonia cases are those with pneumonia occurring $\geq 48 \mathrm{~h}$ after ICU admission and laboratory isolation of PA from any location in the lower respiratory tract. All PA pneumonia diagnoses were cross-validated by trained research physicians. More information on the methods of this analysis are described elsewhere. [6]

Patients were regarded as PA colonized at ICU admission if there was a PA positive screening sample or in case of another PA positive respiratory/skin sample on ICU admission \pm 2 days and if there was no PA infection diagnosed on these days. The incidence density of PA ICU pneumonia was determined using a Cox survival analysis that allows controlling for competing events for the occurrence of ICU pneumonia, in this case ICU discharge/death without ICU pneumonia. The final model yielded 
subdistribution hazard ratios (SHRs) reflecting the relative effect estimates that account for competing events and the other covariates included in the model. The included covariates were age (as a continuous variable), gender, PA colonization at ICU admission, ICU admission specialty (medical vs. surgical) and mechanical ventilation at ICU admission.

Table 1. Baseline characteristics and subdistribution hazard ratio (SHR) for $P$. aeruginosa (PA) ICU pneumonia.

\begin{tabular}{|c|c|c|c|}
\hline & $N(\%)$ or mean (SD) & SHR $(95 \% \mathrm{Cl})$ & $p$ \\
\hline Gender: female $^{1}$ & 1915 (37.6) & $0.73(0.43-1.24)$ & 0.24 \\
\hline $\mathrm{Age}^{2}$ & $59.4(16.1)$ & $0.99(0.98-1.01)$ & 0.43 \\
\hline $\begin{array}{l}\text { Length of stay in days } \\
\text { Median }\end{array}$ & $\begin{array}{l}9.1(11.7) \\
5.0\end{array}$ & - & - \\
\hline Medical admission ${ }^{3}$ & $2176(42.7)$ & $1.34(0.82-2.20)$ & 0.24 \\
\hline \multicolumn{4}{|l|}{$\begin{array}{l}\text { Colonization status at ICU } \\
\text { admission }\end{array}$} \\
\hline - PA - & 2221 (43.6) & ref & ref \\
\hline - PA + & $226(4.4)$ & $8.84(4.96-15.74)$ & $<0.001^{*}$ \\
\hline - Unknown / missing & 2645 (51.9) & $1.04(0.58-1.86)$ & 0.89 \\
\hline ICU mortality & $691(13.6)$ & - & - \\
\hline $\begin{array}{l}\text { Mechanical ventilation at ICU } \\
\text { admission }\end{array}$ & 2591 (50.9) & $5.2(2.70-10.47)$ & $<0.001 *$ \\
\hline ICU pneumonia & $675(13.3)$ & - & - \\
\hline - PA & $68(1.3)$ & & \\
\hline - other confirmed pathogen & $347(6.8)$ & & \\
\hline - unknown pathogen & $260(5.1)$ & & \\
\hline
\end{tabular}

1) male gender is reference category 2) SHR per extra year of age, 3) surgical admission is reference category

* significant at the 0.05 level

- = not measured $/$ not applicable

$\mathrm{SD}=$ standard deviation, $\mathrm{ICU}=$ intensive care unit

\section{RESULTS}

Data were collected from 5093 patients, of whom baseline characteristics can be found in table 1. A total of 2447 patients (48\%) were tested for PA colonization at ICU admission; of those 226 (9.2\%) were PA colonized. A total of 675 (13.3) patients developed ICU pneumonia. Microbiologically confirmed PA ICU pneumonia occurred in 68 patients (1.34\%). In PA colonized patients PA ICU pneumonia occurred in 9.3\% ( $n=21)$; in confirmed non-colonized it occurred in $1.1 \%(n=25)$. The median time to PA ICU 
pneumonia was 7 days. PA colonization was a risk factor for the development of PA ICU pneumonia with a cause-specific hazard ratio (CSHR) of 9.6 (95\% Cl 5.3-17.2, p<0.001). Mechanical ventilation at ICU admission was associated with higher CSHR for developing PA ICU pneumonia (CSHR 2.9; 95\% CI 1.4-6.0; p=0.004). After accounting for competing events, PA colonization at admission remained a risk factor for the development of PA ICU-pneumonia (SHR 8.8, 95\% CI 5.0-15.7, p<0.001, Table 1, Figure 1), as was mechanical ventilation at ICU admission (SHR 5.3, 95\% Cl 2.7-10.5, $\mathrm{p}<0.001$ ).

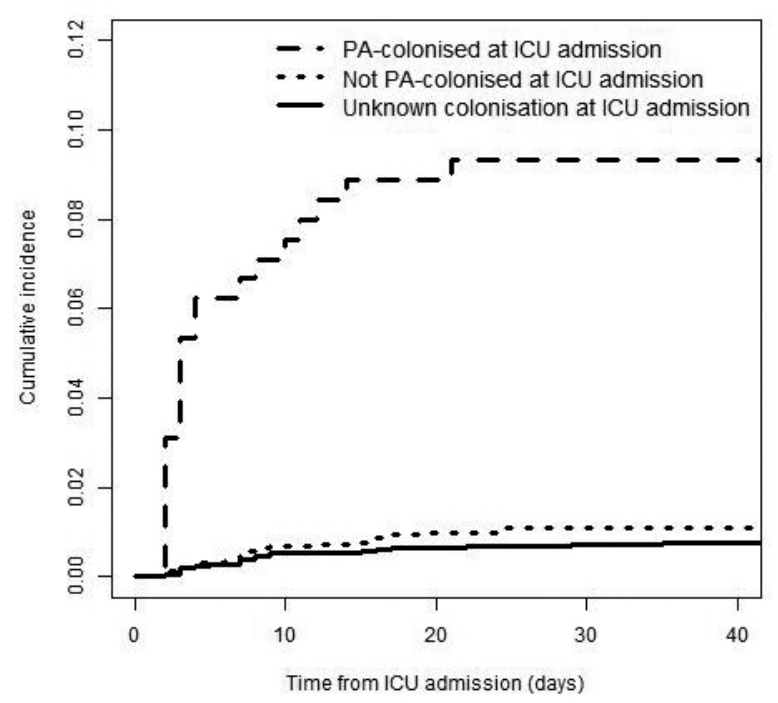

Figure 1. Cumulative incidence function. Cumulative risk of acquiring P. aeruginosa ICU pneumonia

\section{DISCUSSION}

In this study PA colonized ICU patients with a length of stay of $\geq 48$ hours had an almost nine times higher risk of developing PA ICU pneumonia than non-colonized patients. Studies that investigate PA colonization as a risk factor for subsequent PA infection are very scarce, and they do not perform multivariate time-to-event analysis in combination with competing risk analyses. [3], [4], [7]-[9]

This study has several limitations, one of them being a single-center study, another being the fact that only half of the patients were tested for PA colonization at ICU admission. Reasons for not testing included the anticipated short stay on ICU for post- 
surgical patients. Unfortunately, reasons for admission were not recorded and thus we cannot validate this explanation. A second draw-back is the relatively high number of pneumonias caused by unknown pathogens. We cannot rule out that these were caused by (non-cultured) $P$. aeruginosa. However, we performed a sensitivity analyses taking into account pneumonias caused by other and unknown pathogens as competing events, to assess if this changed our final estimates. This was not the case.

Despite the limitations, this study suggests that previous PA colonization contributes to the development of PA ICU pneumonia. Identifying patients at higher risk for developing subsequent infection is important in case that preventive medication becomes available, but also when empirical therapy needs to be started.

\section{CONCLUSION}

In this study the incidence of $P$. aeruginosa ICU pneumonia was $1.34 \%$. Hazard ratios for PA colonized patients compared to non-colonized to develop PA ICU pneumonia were 8.8 .

Funding: This study was funded by the COMBACTE Consortium, which in turn was funded by Innovative Medicines Initiative Joint Undertaking (grant no. 115523). 


\section{REFERENCES}

1. S. Fujitani, H.-Y. Sun, V. L. Yu, and J. A. Weingarten, "Pneumonia due to Pseudomonas aeruginosa: part I: epidemiology, clinical diagnosis, and source.," Chest, vol. 139, no. 4, pp. 909-19, Apr. 2011.

2. R. Gaynes, J. R. Edwards, and National Nosocomial Infections Surveillance System, "Overview of nosocomial infections caused by gram-negative bacilli.," Clin. Infect. Dis., vol. 41, no. 6, pp. 848-54, Sep. 2005.

3. M. H. Kollef et al., "Global prospective epidemiologic and surveillance study of ventilatorassociated pneumonia due to Pseudomonas aeruginosa*.," Crit. Care Med., vol. 42, no. 10, pp. 2178-87, Oct. 2014.

4. L. Fernández-Barat et al., "Intensive care unit-acquired pneumonia due to Pseudomonas aeruginosa with and without multidrug resistance.," J. Infect., vol. 74, no. 2, pp. 142-152, Feb. 2017.

5. L. De Bus et al., "Validity analysis of a unique infection surveillance system in the intensive care unit by analysis of a data warehouse built through a workflow-integrated software application.," J. Hosp. Infect., vol. 87, no. 3, pp. 159-64, Jul. 2014.

6. F. P. Paling et al., "Staphylococcus aureus colonization at ICU admission as a risk factor for developing S. aureus ICU pneumonia," Clin. Microbiol. Infect., vol. 23, no. 1, p. 49.e9-49.e14, Jan. 2017.

7. A. D. Harris et al., "Pseudomonas aeruginosa Colonization in the Intensive Care Unit: Prevalence, Risk Factors, and Clinical Outcomes," Infect. Control Hosp. Epidemiol., vol. 37, no. 5, pp. 544-548, May 2016.

8. A. G. Venier et al., "Identifying new risk factors for Pseudomonas aeruginosa pneumonia in intensive care units: experience of the French national surveillance, REA-RAISIN.," J. Hosp. Infect., vol. 79, no. 1, pp. 44-8, Sep. 2011.

9. J. Vallés et al., "Patterns of colonization by Pseudomonas aeruginosa in intubated patients: a 3-year prospective study of 1,607 isolates using pulsed-field gel electrophoresis with implications for prevention of ventilator-associated pneumonia.," Intensive Care Med., vol. 30, no. 9, pp. 1768-75, Sep. 2004. 



\section{PART TWO}

PROSPECTIVE 


$$
\pi \pi
$$




\title{
Rationale and design of ASPIRE- \\ ICU: a prospective cohort study on the incidence and predictors of Staphylococcus aureus and Pseudomonas aeruginosa pneumonia in the ICU
}

\section{CHAPTER}

\author{
Fleur P. Paling ${ }^{1}$ \\ Darren P.R. Troeman ${ }^{1}$ \\ Martin Wolkewitz ${ }^{2}$ \\ Rubana Kalyani ${ }^{3}$ \\ Daniël R. Prins ${ }^{1}$ \\ Susanne Weber ${ }^{2}$ \\ Christine Lammens ${ }^{4}$ \\ Leen Timbermont ${ }^{4}$ \\ Herman Goossens ${ }^{4}$ \\ Surbhi Malhotra-Kumar ${ }^{4}$ \\ Frangiscos Sifakis ${ }^{5}$ \\ Marc J.M. Bonten ${ }^{1,6}$ \\ Jan A.J.W. Kluytmans ${ }^{1,7}$
}

Published in: BMC Infectious Diseases, 2017 Sep 25;17(1):643.

doi: 10.1186/s12879-017-2739-4

\footnotetext{
${ }^{1}$ Julius Center for Health Sciences and Primary Care, University Medical Center Utrecht, Utrecht, Netherlands

${ }^{2}$ Institute for Medical Biometry and Statistics, Faculty of Medicine and Medical Center,

University of Freiburg, Germany

${ }^{3}$ MedImmune, Gaithersburg, Maryland, U.S.A.

${ }^{4}$ Laboratory of Medical Microbiology, Vaccine \& Infectious Disease Institute (VAXINFECTIO), University of Antwerp, Wilrijk, Belgium

${ }^{5}$ AstraZeneca Pharmaceuticals LP, Gaithersburg, Maryland, U.S.A.

${ }^{6}$ Department of Medical Microbiology, University Medical Center Utrecht, Utrecht, Netherlands

${ }^{7}$ Amphia Hospital, Breda, The Netherlands
} 


\section{ABSTRACT}

Background: The epidemiology of ICU pneumonia caused by Staphylococcus aureus (S. aureus) and Pseudomonas aeruginosa (P. aeruginosa) is not fully described, but is urgently needed to support the development of effective interventions. The objective of this study is to estimate the incidence of $S$. aureus and $P$. aeruginosa ICU pneumonia and to assess its association with patient-related and contextual risk factors.

Methods: ASPIRE-ICU is a prospective, observational, multi-center cohort study nested within routine surveillance among ICU patients in Europe describing the occurrence of $S$. aureus and $P$. aeruginosa ICU pneumonia. Two thousand $(2,000)$ study cohort subjects will be enrolled ( $50 \%$ S. aureus colonized) in which specimens and data will be collected. Study cohort subjects will be enrolled from a larger surveillance population, in which basic surveillance data is captured. The primary outcomes are the incidence of $S$. aureus ICU acquired pneumonia and the incidence of $P$. aeruginosa ICU acquired pneumonia through ICU stay.

The analysis will include advanced survival techniques (competing risks and multistate models) for each event separately as well as for the sub-distribution of ICU pneumonia to determine independent association of outcomes with risk factors.. A risk prediction model will be developed to quantify the risk for acquiring $S$. aureus or $P$. aeruginosa ICU pneumonia during ICU stay by using a composite score of independent risk factors.

Discussion: The diagnosis of pathogen-specific ICU pneumonia is difficult, however, the criteria used in this study are objective and comparable to those in the literature.

Trial registration: This study is registered on clinicaltrials.gov under identifier NCT02413242. 


\section{BACKGROUND}

Patients hospitalized in the Intensive Care Unit (ICU) are at risk of acquiring pneumonia, especially if they are mechanically ventilated. Despite extensive efforts, ICU-acquired pneumonia continues to be one of the most frequently occurring complications in the ICU and increases morbidity as well as mortality [1], [2]. Accurately describing and predicting the occurrence of ICU pneumonia is difficult as a result of inconsistencies in the case definition and surveillance methods in different countries [1], [3]. The need for an accurate and standardized prognosis is important considering current interventions are preventive rather than therapeutic [4]. As part of the COMBACTE consortium (COMBatting AntibiotiC resisTance in Europe), two monoclonal antibodies (mAbs) are currently being developed that target $S$. aureus and $P$. aeruginosa, respectively [5], [6]. Both bacteria are frequently occurring causative pathogens of ICU pneumonia and administration of a mAb may prevent the development of ICU pneumonia with these pathogens [7], [8]. Prospective studies can assess possible risk factors that can identify subsets of patients that may benefit most from these interventions. Thus, the goal of this study is to systematically assess the impact of patient-related and contextual factors on the incidence of $S$. aureus and $P$. aeruginosa ICU pneumonia in Europe and to identify the patient subgroups that are at greater risk for disease and bear a disproportionate disease burden. These objectives will directly contribute to the sample size and feasibility calculations for clinical trial design of the mAb interventions [8]. 


\section{METHODS}

\section{Objectives}

The primary objectives of this study are to determine the incidence of:

(1) S. aureus ICU pneumonia through ICU stay; and

(2) P. aeruginosa ICU pneumonia through ICU stay; and

their independent associations with patient-related factors (e.g. colonization status, baseline serum antibody levels against $S$. aureus or $P$. aeruginosa antigens) and contextual factors.

The key secondary objectives are to develop risk prediction models to quantify the risk of acquiring (i) S. aureus or (ii) P. aeruginosa ICU pneumonia during ICU stay, by using a composite score of independent risk factors identified through primary objective 1 and 2. Other secondary and exploratory objectives can be found on ClinicalTrials.gov, under identifier NCT02413242, or in the online supplemental material (S.01).

\section{Study design}

ASPIRE-ICU (Advanced understanding of Staphylococcus aureus and Pseudomonas aeruginosa Infections in EuRopE - Intensive Care Units) is a multi-center, prospective, observational cohort study nested within ongoing routine surveillance among ICU patients in Europe. The study is composed of two study populations, the surveillance population and study cohort population. The study cohort is nested within the larger surveillance population; this means that all data and specimens collected specifically for study cohort participants is in addition to data already captured by ways of surveillance. An overview of the schedule of procedures, including all sample collection types and time points can be found in the online supplemental material (Table S.02).

\section{Study populations and recruitment}

\section{Surveillance population}

Patients eligible to participate in the surveillance population must be on mechanical ventilation (MV) upon or (expected to be) within 24 hours after ICU admission and have an expected length of stay (LOS) of at least 48 hours. Patients with an expected ICU stay of less than 48 hours are at a lower risk for developing ICU infections since this population is generally healthier, without significant comorbidities and shorter in the ICU. 
The surveillance population are considered to be the source population from which the study cohort subjects are derived. No informed consent is required for participation in the surveillance population, but depending on local legislation, patients of the surveillance population will receive information (i.e. leaflet/flyer) on the ASPIRE-ICU study and are able to deny use of their de-identified data for scientific purposes.

\section{Study cohort population}

Surveillance patients that meet the eligibility criteria described below for the study cohort population will be enrolled. 2,000 study cohort subjects are required to meet the objectives of this study. Study cohort subjects are approached for informed consent based on their S. aureus colonization status at ICU admission. Subjects will be enrolled in a 1:1 ratio of $S$. aureus colonized subjects to non $S$. aureus colonized subjects with 1,000 subjects in each stratum. A similar temporal distribution of enrolled $S$. aureus colonized and non S. aureus -colonized subjects will be managed by selecting the first non-colonized subject after including a colonized subject. For subjects unable to provide consent for any reason, a legally accepted representative may consent on the subject's behalf at the time of enrollment.

Inclusion criteria for study cohort

1. Participant is 18 years or older at the time of enrollment.

2. Participant is on mechanical ventilation at ICU admission, or is (expected to be) within 24 hours thereafter, based on investigator's judgment.

3. Expected stay in ICU is 48 hours or longer based on investigator's judgment.

4. S. aureus colonization status is known within 72 hours after start of first episode of mechanical ventilation and according to the result, the patient qualifies for enrollment.

5. Written informed consent from subject / legally accepted representative within 72 hours after start of first episode of mechanical ventilation.

Exclusion criteria for study cohort

1. Previous participation as a subject in the study cohort of this study.

2. Simultaneous participation of the subject in any preventive experimental study into anti-staphylococcus or anti-pseudomonas aeruginosa interventions.

3. Expected death (moribund status) within 48h, or ICU discharge of the participant within $24 \mathrm{~h}$, at the moment of informed consent. 


\section{Study outcome definitions}

Considering that the definition of our primary outcome, ICU pneumonia caused by $S$. aureus or $P$. aeruginosa, is very extensive, we would like to refer to the supplemental material (S.03) containing the full definition. In summary, ICU pneumonia is defined as pneumonia occurring $\geq 48 \mathrm{~h}$ after admission to the ICU and is confirmed by a new or worsening infiltrate on chest X-ray or CT-thorax. Furthermore, the patient must fulfill specific clinical criteria (for example abnormal temperature, production of sputum, auscultatory abnormalities, acute changes in the ventilatory support system), in addition to at least 1 microbiological criterion (positive respiratory specimen, blood culture, pleural fluid aspirate or lung tissue culture).

\section{Site selection}

In total, 30 sites in 12 countries were selected from sources including but not limited to the COMBACTE CLIN-Net and LAB-Net databases [7]. To ensure the pan-European continent is represented, there is at least one country included from the Northern / Southern / Eastern and Western region of Europe. The selected sites adequately balance different factors such as geography, background antibiotic resistance prevalence, etc.. To ensure enrollment is met within the expected timelines, back-up sites were selected for various reasons (i.e. low enrollment numbers, decline further participation) that can supplement or replace primary sites selected. For each site also a local laboratory was selected to participate in the study.

A Site Selection Committee selected sites and laboratories based on pre-defined criteria in the Site Selection Plan. Assessment of these criteria was aided by site feasibility questionnaires. Participating ICUs must have a routine $S$. aureus screening protocol in order to be selected for participation.

Screening should consist of a minimum of one nasal swab and one lower respiratory tract (LRT) sample analyzed locally on the day of ICU admission. The LRT sample is defined as the collection of an endotracheal aspirate (ETA) sample, or, if an ETA cannot be collected, a sputum sample may be taken. As an exception, for routine $S$. aureus colonization screening at ICU admission only, if both the ETA and sputum cannot be collected, a throat swab may be taken. 


\section{Statistical analysis}

Sample size calculation

Sample size calculations are based on the expected incidence precision of $S$. aureus ICU pneumonia and $P$. aeruginosa ICU pneumonia, since the primary objective of the study is to identify the patient groups most at risk for this outcome.

Assuming an incidence of S. aureus ICU pneumonia of $12.5 \%$ and $1.5 \%$ in the S. aureus colonized group and non $S$. aureus colonized group respectively, this would result in an overall incidence of $7 \%$ within the 2,000 study cohort subjects, or $140 \mathrm{~S}$. aureus ICU pneumonia endpoints[9]. The overall incidence estimate would have precision of $1.12 \%$ (95\% confidence interval $[\mathrm{Cl}]$ : 5.9\%-8.12\%, using normal approximation).

For $P$. aeruginosa ICU pneumonia, the overall incidence is estimated regardless of colonization status at ICU admission. Assuming an overall incidence of $P$. aeruginosa ICU pneumonia of $2.5 \%$ within the 2,000 study cohort subjects, this would result in 50 $P$. aeruginosa ICU pneumonia endpoints. The overall incidence estimate would have precision of $0.68 \%$ (95\% $\mathrm{Cl} 1.82 \%-3.18 \%$, using normal approximation).

\section{Planned analysis}

The primary analysis will evaluate the incidence density of $S$. aureus or $P$. aeruginosa ICU pneumonia; its calculation will depend on time from admission (for outcome ICU pneumonia) or time from ventilation (for outcome ventilator-associated pneumonia).

For the primary and secondary objectives, advanced survival techniques (competing risks and multistate models) will be applied. Discharge and death will be considered as competing events for ICU pneumonia. Adapted Cox regression models will be applied for each event separately as well as for the sub-distribution of ICU pneumonia. The clustering of the data (readmission, patients within ICU, country) will be acknowledged using shared frailty methodology, stratification or robust variance. The timedependency of cumulative hazards and incidences will be graphically displayed, by risk factors of interest. Hazard ratios with $95 \% \mathrm{Cl}$ will be calculated univariately and selected for the multivariate model using an established Akaike's information criterion for model selection. A risk prediction model will be developed to quantify the risk for acquiring $S$. aureus or $P$. aeruginosa ICU pneumonia during ICU stay by using a composite score of independent risk factors. 
Quality assurance

Data will be entered in a web-based electronic data capture system that was designed for ASPIRE-ICU. The study site will enter data in the electronic data capture system from the subject's source documents (i.e. medical chart). Information linking the subject ID to the subject's medical file (only applicable for study cohort subjects) will be kept in a secure place at the participating study site.

Monitoring will include $100 \%$ source data verification for the first three enrolled study cohort subjects at each site, and then $10 \%$ of the remaining enrolled study cohort subjects.

\section{DISCUSSION}

This manuscript describes the objectives and design of ASPIRE-ICU, an observational cohort study addressing risk factors for $S$. aureus and $P$. aeruginosa ICU pneumonia. Certain choices have been made in the design that warrant mention and further discussion.

\section{Definition of pathogen-specific pneumonia}

The definition of $S$. aureus or $P$. aeruginosa ICU pneumonia is based on pre-defined pneumonia criteria in combination with the presence of the bacterium in an appropriate sample around the time of diagnosis, which are aligned with endpoints being used for two randomized controlled trials for prevention of $S$. aureus and $P$. aeruginosa pneumonia[8]. Thus, in case of cultures that yield multiple possible pathogens, it may be that the diagnosis of $S$. aureus or $P$. aeruginosa pneumonia is made incorrectly. The distinction between colonization and infection is sometimes difficult to assess. There is however no 'reference standard' to reliably assess the causative pathogen[3]. We have contemplated quantitative cultures in all pneumonia patients, but it was not feasible to implement this at each site. Quantitative measurements will however be applied on study samples received by the central laboratory, and thus will provide additional retrospective information. Furthermore, other outcomes, such as mortality will provide objective outcome information in addition to a diagnosis of pneumonia.

\section{Enrichment strategy}

In this study, $50 \%$ of the study cohort population is S. aureus colonized at ICU admission to 'enrich' the study population with $S$. aureus carriers (in nose or lower respiratory tract), while this naturally occurs in approximately $20-25 \%$ of the ICU population [10]. This was chosen as $S$. aureus carriage is being studied as one of the main known risk 
factors for subsequent $S$. aureus disease, thus without enrichment, the population needed for equal precision would be much larger [9], [11], [12]. However, the limitation of this choice is that one can argue that a population as such is not representative of the general ICU population, thus incidence estimation as well as assessing risk factors for $P$. aeruginosa ICU pneumonia may be suboptimal. We acknowledge this, and for this reason the surveillance population was included. Their data will allow distribution of baseline factors with the study cohort in an effort to assess the ubiquity of results across both groups. Furthermore, it can serve to identify potential bias between participants and non-participants.

Considering that $P$. aeruginosa colonization at ICU admission is relatively rare and that colonization often occurs after ICU admission, no recruitment selection for $P$. aeruginosa colonized subjects will take place[13].

\section{Routine surveillance}

This study utilizes routine $S$. aureus screening conducted at each participating site as the basis for eligibility assessment. For consistency of screening results across sites, all swabs will be plated under protocol on the same chromogenic agar plates (Colorex agar, bioTRADING Benelux) provided by the ASPIRE-ICU study team. In the original protocol, a nose swab and ETA sample (sputum sample if non-intubated) are collected at screening. However, soon after study start, the protocol was amended to include a throat sample as a LRT sample, in case ETA and sputum were both not feasible.

\section{Future perspectives}

In this era of increasing antimicrobial resistance, the research field is steadily exploring other therapies, for example prophylactic therapies such as antibody-based preventive measures. A potential advantage of monoclonal antibodies (mAbs) is that they will not encourage bacterial resistance to the same extent as antibiotics, and may even augment antibiotic effectiveness [14]. This study was designed in part to provide crucial information on the incidence, patient-related and contextual factors of ICU pneumonia caused by $S$. aureus and $P$. aeruginosa, but also inform the design of future phase III trials, that will investigate $\mathrm{mAbs}$ effectiveness against $S$. aureus and $P$. aeruginosa. Two large COMBACTE phase II trials, SAATELLITE (A Human Monoclonal Antibody Against Staphylococcus aureus Alpha Toxin in Mechanically Ventilated Adult Subjects) and EVADE (Effort to Prevent Nosocomial Pneumonia Caused by Pseudomonas aeruginosa in Mechanically Ventilated Subjects) have already started [8]. SAATELLITE investigates the effect of MEDI4893, a mAb targeting S. aureus alpha toxin and EVADE studies MEDI3902, 
which is another mAb that simultaneously targets PcrV and Psl on the surface of the $P$. aeruginosa bacterium, in subjects at risk for ICU pneumonia [5], [6], [8]. These targeted therapies, if proven effective, may be used in the future for patients at highest risk. This study will help to identify the subset of patients that will likely benefit most.

\section{CONCLUSION}

This epidemiological cohort study on $S$. aureus and $P$. aeruginosa ICU pneumonia aims to add significant information to the literature on predictors for this event. This will help refine the design of Phase III trials and may benefit patients at risk for nosocomial infections, by providing protective measures.

\section{List of abbreviations}

$\begin{array}{ll}\text { ASPIRE-ICU } & \begin{array}{l}\text { Advanced understanding of Staphylococcus aureus and } \\ \text { Pseudomonas aeruginosa Infections in EuRopE } \\ \mathrm{CI}\end{array} \\ \text { COMfidence interval } \\ \text { COMBACTE } & \text { Endotracheal aspirate } \\ \text { ETA } & \text { Intensive Care Unit } \\ \text { ICU } & \text { Length of stay } \\ \text { LOS } & \text { Lower respiratory tract } \\ \text { LRT } & \text { Monoclonal antibody } \\ \text { mAb } & \text { Pseudomonas aeruginosa } \\ \text { P. aeruginosa } & \text { Staphylococcus aureus } \\ \text { S. aureus } & \end{array}$

Acknowledgments: We would like to thank the following people for their contributions while writing this study protocol: Stephan Harbarth, Mark Eickhoff, Bruno François, Frank Coenjaerts, Uwe Völker, Barbara Bröker, Andreas Peschel, Willem Van Wamel, Gerard Lina, Jos Van Strijp, Samir Kumar-Singh, Antonio Oliver, Christian Van Delden, Thilo Kohler, Tom van der Poll, Craig Maclean.

Funding: This research project receives support from the Innovative Medicines Initiative Joint Undertaking under grant agreement no. 115523 and 115737 resources which are composed of financial contribution from the European Union Seventh Framework Programme (FP7/2007-2013) and EFPIA companies in kind contribution. 


\section{REFERENCES}

1. J. Chastre and J.-Y. Fagon, "Ventilator-associated Pneumonia," Am. J. Respir. Crit. Care Med., vol. 165, no. 7, pp. 867-903, Apr. 2002.

2. J. L. Vincent et al., "The prevalence of nosocomial infection in intensive care units in Europe. Results of the European Prevalence of Infection in Intensive Care (EPIC) Study. EPIC International Advisory Committee.," JAMA, vol. 274, no. 8, pp. 639-44, 1995.

3. I. S. Douglas, "New diagnostic methods for pneumonia in the ICU," Curr. Opin. Infect. Dis., vol. 29, no. 2, pp. 197-204, Apr. 2016.

4. B. François, H. S. Jafri, and M. Bonten, "Alternatives to antibiotics.," Intensive Care Med., vol. 42, no. 12, pp. 2034-2036, Dec. 2016.

5. L. Hua et al., "Assessment of an anti-alpha-toxin monoclonal antibody for prevention and treatment of Staphylococcus aureus-induced pneumonia.," Antimicrob. Agents Chemother., vol. 58, no. 2, pp. 1108-17, 2014.

6. A. DiGiandomenico et al., "Identification of broadly protective human antibodies to Pseudomonas aeruginosa exopolysaccharide Psl by phenotypic screening.," J. Exp. Med., vol. 209, no. 7, pp. 1273-87, Jul. 2012.

7. T. Kostyanev et al., "The Innovative Medicines Initiative's New Drugs for Bad Bugs programme: European public-private partnerships for the development of new strategies to tackle antibiotic resistance.," J. Antimicrob. Chemother., vol. 71, no. 2, pp. 290-5, Feb. 2016.

8. B. François et al., "The SAATELLITE and EVADE Clinical Studies Within the COMBACTE Consortium: A Public-Private Collaborative Effort in Designing and Performing Clinical Trials for Novel Antibacterial Drugs to Prevent Nosocomial Pneumonia: Table 1.," Clin. Infect. Dis., vol. 63, no. suppl 2, pp. S46-S51, Aug. 2016.

9. F. P. Paling et al., "Staphylococcus aureus colonization at ICU admission as a risk factor for developing S. aureus ICU pneumonia," Clin. Microbiol. Infect., vol. 23, no. 1, p. 49.e9-49. e14, Jan. 2017.

10. H. F. L. Wertheim et al., "The role of nasal carriage in Staphylococcus aureus infections.," Lancet Infect. Dis., vol. 5, no. 12, pp. 751-62, Dec. 2005.

11. P. D. Ziakas, T. Anagnostou, and E. Mylonakis, "The prevalence and significance of methicillinresistant Staphylococcus aureus colonization at admission in the general ICU Setting: a metaanalysis of published studies.," Crit. Care Med., vol. 42, no. 2, pp. 433-44, Feb. 2014. 
12. K. A. Davis, J. J. Stewart, H. K. Crouch, C. E. Florez, and D. R. Hospenthal, "Methicillin-resistant Staphylococcus aureus (MRSA) nares colonization at hospital admission and its effect on subsequent MRSA infection.," Clin. Infect. Dis., vol. 39, no. 6, pp. 776-82, Sep. 2004.

13. M. H. Kollef et al., "Global prospective epidemiologic and surveillance study of ventilatorassociated pneumonia due to Pseudomonas aeruginosa.," Crit. Care Med., vol. 42, no. 10, pp. 2178-87, Oct. 2014.

14. C. Saylor, E. Dadachova, and A. Casadevall, "Monoclonal antibody-based therapies for microbial diseases.," Vaccine, vol. 27 Suppl 6, pp. G38-46, Dec. 2009. 


\section{SUPPLEMENTARY MATERIAL}

S01. Supplementary appendix 1: Complete list of objectives and endpoints.

S02. Supplementary table 2: Schedule of procedures.

S03. Supplementary appendix 3: Complete definition of study endpoints. 
S01. Supplementary appendix 1. Complete list of objectives and endpoints

\section{Objectives}

\section{Primary objectives}

1. To determine the incidence of ICU pneumonia caused by S. aureus through ICU stay and its independent association with patient-related factors (e.g. baseline serum antibody levels against $S$. aureus alpha toxin [binding and functional], S. aureus colonization in nose/ETA) and contextual factors.

2. To determine the incidence of ICU pneumonia caused by $P$. aeruginosa through ICU stay and its independent association with patient-related factors (e.g. baseline $P$. aeruginosa serum antibody levels against Psl and PcrV [binding and functional], $P$. aeruginosa colonization in peri-anal region/ETA), and contextual factors.

\section{Secondary objectives}

1. To develop a risk prediction model to quantify the risk of acquiring S. aureus ICU pneumonia during ICU stay, by using a composite score of independent risk factors identified through primary objective 1.

2. To develop a risk prediction model to quantify the risk of acquiring $P$. aeruginosa ICU pneumonia during ICU stay, by using a composite score of independent risk factors identified through primary objective 2 .

3. Diagnostic

a. To determine if a rapid, PCR-based diagnostic is as sensitive and specific as traditional culture to identify $S$. aureus colonization .

b. To determine if a rapid, PCR-based diagnostic is as sensitive and specific as traditional culture to identify $P$. aeruginosa colonization.

4. To determine the incidence of all-cause ICU pneumonia (and VAP specifically) and to describe its temporal distribution in relation to hospitalization in the ICU.

5. To assess the incidence of ICU pneumonia attributable to $S$. aureus stratified by methicillin susceptibility (methicillin-susceptible S. aureus [MSSA] and methicillinresistant S. aureus [MRSA]).

6. To assess the incidence of ICU pneumonia attributable to $P$. aeruginosa stratified by multi-drug-resistance (multi-drug resistant $P$. aeruginosa [MDR-PA] and susceptible $P$. aeruginosa [S-PA]).

7. To assess the incidence of ICU acquired bacteremia by etiologic agent (S. aureus, $P$. aeruginosa and/or for all other clinically relevant other pathogens combined) and to describe its temporal distribution in relation to hospitalization in the ICU. 
8. To assess the independent association of $S$. aureus nasal colonization with all-cause mortality and risk of $S$. aureus infection.

9. To assess the independent association of $P$. aeruginosa peri-anal colonization with all-cause mortality and risk of $P$. aeruginosa infection.

10. To assess expression of known (AT, ClfA, SpA, ISDH etc.) and other virulence factors, as identified by transcriptomics/proteomics experiments (toxinome studies) in $S$. aureus isolates associated with colonization or ICU pneumonia.

11. To assess the gene sequence of $S$. aureus known virulence factors (AT, ClfA, SpA, ISDH etc.) and of those identified in toxinome studies in $S$. aureus isolates associated with colonization or ICU pneumonia.

12. To assess $P$. aeruginosa isolates associated with colonization or ICU pneumonia for variations in PcrV, Psl, and associated genes.

13. To assess PcrV, Psl, and other virulence factors expression under anti-infective pressure in in vitro biofilm model and in VAP and pneumonia animal models.

14. Biomarkers:

a. To explore the role of antibodies against S. aureus virulence factors (for example clumping factor A [ClfA], Staphylococcal protein A [SpA], and [ISDH] and those identified in toxinome studies) as potential biomarkers associated with $S$. aureus infection.

b. To assess the independent association between baseline serum antibody levels against the Pseudomonas aeruginosa PcrV and polysaccharide synthesis locus (Psl) virulence factors and $P$. aeruginosa infection.

c. To assess the independent association between host biomarkers (e.g. baseline antibody levels against pathogen virulence factors, inflammatory markers, differentially expressed RNA molecules and proteins), the occurrence of ICU pneumonia and clinical outcomes among cases of ICU pneumonia.

d. To assess the independent association between pathogen biomarkers (e.g. presence of $P$. aeruginosa or $S$. aureus virulence factors) and clinical outcomes among cases of $P$. aeruginosa or $S$. aureus infections.

\section{Exploratory objectives}

1. To describe magnitude of healthcare utilization associated with $S$. aureus ICU pneumonia (e.g. length of ICU stay and duration of mechanical ventilation).

2. To describe magnitude of healthcare utilization associated with $P$. aeruginosa ICU pneumonia (e.g. length of ICU stay and duration of mechanical ventilation). 
3. To determine strain characteristics of $S$. aureus and $P$. aeruginosa isolates from ICU pneumonia cases

a. Determinants of resistance, virulence and other relevant genes

b. To assess the proportion of S. aureus isolates in which the AT-gene is present on the genome.

c. Monitor prevalence of S. aureus clonal types associated with colonization and ICU pneumonia cases.

d. To assess the proportion of $P$. aeruginosa isolates on which the Pseudomonas aeruginosa PcrV or polysaccharide synthesis locus (Psl) gene is present.

4. To explore the role of antibodies against Gram-positive and Gram-negative bacterial virulence factors as biomarkers.

5. To identify independent risk factors for acquiring S. aureus colonization during ICU stay.

6. To identify independent risk factors for acquiring $P$. aeruginosa colonization during ICU stay.

7. To compare participating study sites and sites participating in routine HAI surveillance (e.g., ECDC HAI-Net or other external data sources) to further inform external validity of results.

\section{Endpoints}

Primary endpoints

1. Incidence of $S$. aureus ICU pneumonia in subjects until ICU discharge.

2. Incidence of $P$. aeruginosa ICU pneumonia in subjects until ICU discharge.

\section{Secondary endpoints}

1. Prevalence of S. aureus / P. aeruginosa colonization at ICU admission in subjects.

2. Incidence of all cause ICU pneumonia in subjects until ICU discharge.

3. Incidence of $S$. aureus ICU pneumonia stratified by MRSA vs. MSSA.

4. Incidence of $P$. aeruginosa ICU pneumonia stratified by MDR-PA vs. S-PA.

5. Incidence of ICU bacteremia per etiologic agent (in case of $S$. aureus and/or $P$. aeruginosa and for all clinically relevant other pathogens) in subjects until ICU discharge.

6. All-cause mortality throughout ICU stay.

7. All-cause mortality at day 30 after ICU admission.

8. All-cause mortality at day 90 after ICU admission.

9. Time to $S$. aureus ICU pneumonia until ICU discharge.

10. Time to $P$. aeruginosa ICU pneumonia until ICU discharge. 
11. Time to all cause ICU pneumonia until ICU discharge.

12. Time to all cause ICU bacteremia until ICU discharge.

13. Time to death of any cause up to 90 days following ICU admission or until ICU discharge.

\section{Exploratory endpoints}

1. Magnitude of healthcare utilization as measured by:
a. Duration of ICU stay including readmissions
b. Days on mechanical ventilation
c. Days of antibiotic usage
d. Duration of hospital stay, including readmissions

2. Incidence of $S$. aureus colonization after ICU admission but prior to ICU pneumonia.

3. Incidence of $P$. aeruginosa colonization after ICU admission but prior to ICU pneumonia. 


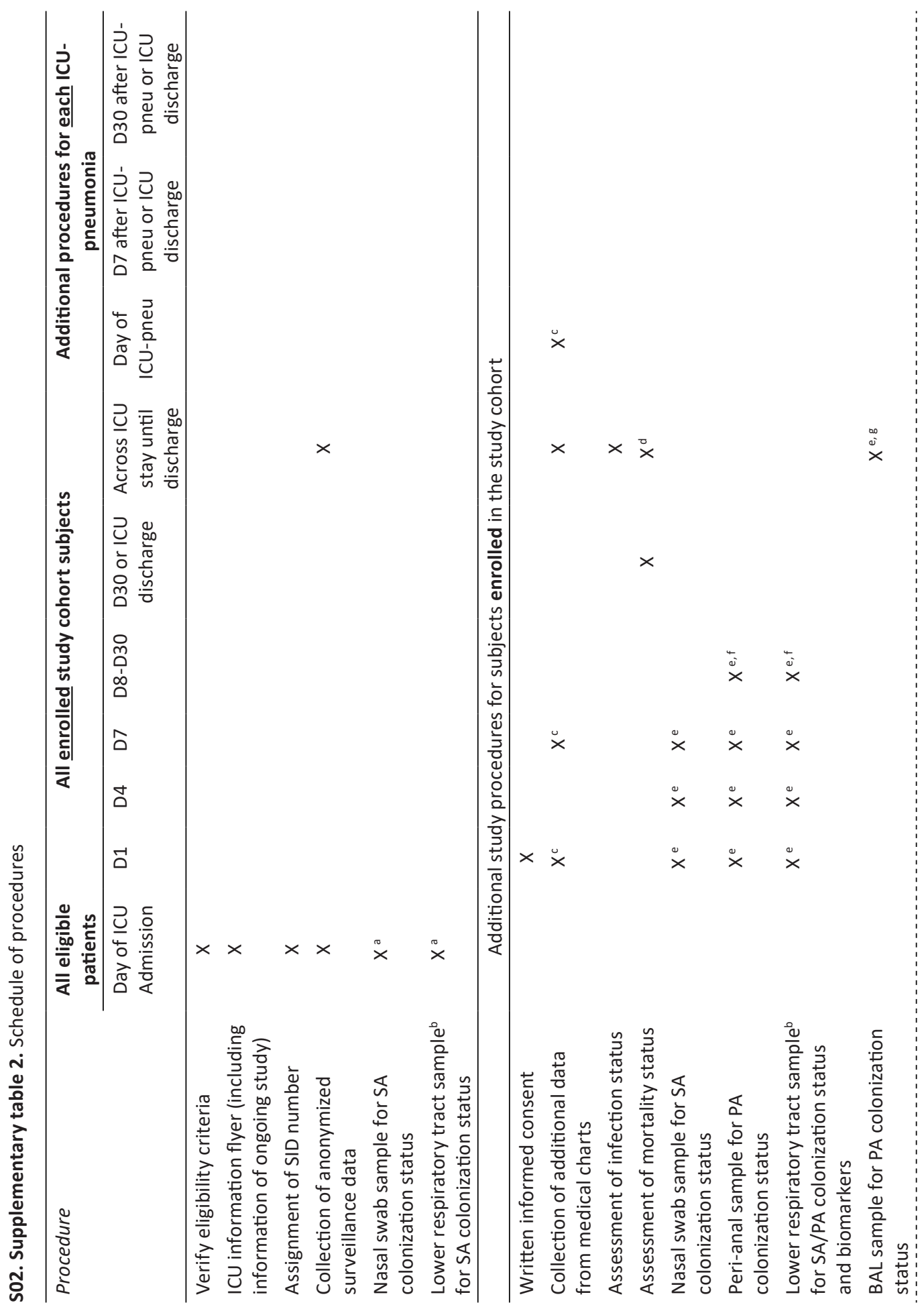




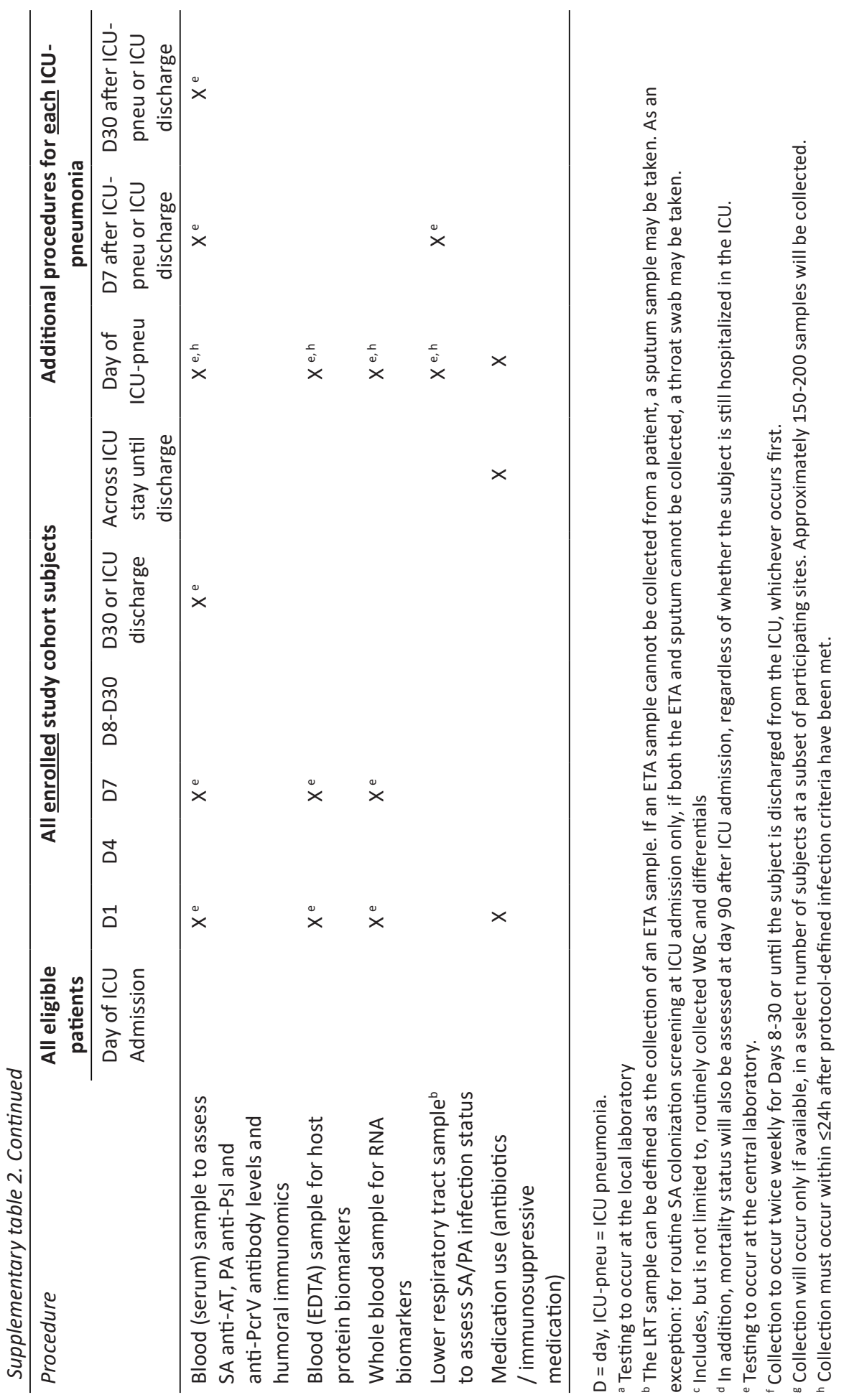


S03. Supplementary appendix 3. Complete definition of study endpoints

1. ICU pneumonia in mechanically ventilated patients

Patient should demonstrate the following new onset of symptoms/signs deemed not due to any overt non-infectious causes.

\section{a. Radiographic criteria:}

New or worsening infiltrate consistent with pneumonia on chest X-ray or CT-thorax obtained within 24 hours of the event (diagnosed by a qualified radiologist).

\section{AND}

\section{b. Clinical criteria:}

At least $\underline{\mathbf{2}}$ of the following minor or $\underline{\mathbf{1}}$ major respiratory sign or symptom of new onset:

Minor criteria:

- Systemic signs of infection (one or more of the following): Abnormal temperature (oral or tympanic temperature $>38^{\circ} \mathrm{C}$ or a core temperature $\geq 38.3^{\circ} \mathrm{C}$ or hypothermia, defined as a core body temperature of $<35^{\circ} \mathrm{C}$ ), and/or abnormal WBC (WBC count $>10,000$ cells $/ \mathrm{mm}^{3}$, WBC count $<4500$ cells $/ \mathrm{mm}^{3}$, or $>15 \%$ band neutrophils)

- Production of purulent endotracheal secretions

- Auscultatory findings consistent with pneumonia/pulmonary consolidation (e.g. rales, rhonchi, bronchial breath sounds, dullness to percussion)

Major criteria: Acute changes made in the ventilatory support system to enhance oxygenation, as determined by:

- $\mathrm{PaO}_{2} / \mathrm{FiO}_{2}$ ratio $<240 \mathrm{mmHg}$, or

- A decrease in $\mathrm{PaO}_{2} / \mathrm{FiO}_{2}$ by $\geq 50 \mathrm{mmHg}$ 
2. ICU pneumonia in not mechanically ventilated patients

Patient should demonstrate the following new onset of symptoms/signs deemed not due to any overt non-infectious causes.

\section{a. Radiographic criteria:}

New or worsening infiltrate consistent with pneumonia on chest X-ray or CT-thorax obtained within 24 hours of the event (diagnosed by qualified radiologist)

\section{AND}

\section{b. Clinical criteria:}

At least $\underline{\mathbf{2}}$ of the following minor or $\underline{\mathbf{1}}$ major respiratory signs or symptoms:

Minor criteria:

- Systemic signs of infection: Abnormal temperature (oral or tympanic temperature $>38^{\circ} \mathrm{C}$ or a core temperature $\geq 38.3^{\circ} \mathrm{C}$ or hypothermia, defined as a core body temperature of $<35^{\circ} \mathrm{C}$ ), and/or abnormal WBC (WBC count $>10,000$ cells $/ \mathrm{mm}^{3}$, WBC count $<4500$ cells $/ \mathrm{mm}^{3}$, or $>15 \%$ band neutrophils)

- A new onset of cough (or worsening of cough)

- Production of purulent sputum

- Physical examination findings consistent with pneumonia/pulmonary consolidation such as auscultatory findings (e.g. rales, rhonchi, bronchial breath sounds), dullness to percussion, or pleuritic chest pain

- Dyspnea, tachypnea (respiratory rate > 30 breaths/minute), or hypoxemia defined as:

- $\mathrm{O}_{2}$ saturation $<90 \%$ or $\mathrm{PaO}_{2}<60 \mathrm{mmHg}$ on room air if lower than baseline, or

- A need to initiate or increase sustained ( $\geq 3$ hours) supplemental oxygen to maintain pre-event baseline $\mathrm{O}_{2}$ saturations

Major criteria:

A need to initiate non-invasive mechanical ventilation or re-initiate invasive mechanical ventilation because of respiratory failure or worsening of respiratory status 
3. S. aureus ICU pneumonia in mechanically ventilated patients

Patient should meet all criteria as described for ICU pneumonia in mechanically ventilated patients (S.03.1) AND at least 1 of the following microbiological criteria:

- Respiratory specimen (obtained within 72 hours of onset of the event) is positive for $S$. aureus by culture. Includes a specimen of respiratory secretions obtained by endotracheal aspiration or by bronchoscopy with bronchoalveolar lavage (BAL) or protected-specimen brush (PSB) sampling in intubated subjects

- Blood culture positive for S. aureus (and no apparent primary source of infection outside the lung)

- Pleural fluid aspirate or lung tissue culture positive for S. aureus during episode of pneumonia (only if obtained as part of the subject's necessary clinical management or post-mortem)

4. S. aureus ICU pneumonia in not mechanically ventilated patients

Patient should meet all criteria as described for ICU pneumonia in not mechanically ventilated patients (S.03.2) AND at least 1 of the following microbiological criteria:

- Respiratory specimen (obtained within 72 hours of onset of the event) is positive for S. aureus by culture. Includes either expectorated sputum or (only if obtained as part of the subject's necessary clinical management or post-mortem) a specimen of respiratory secretions obtained by bronchoscopy with BAL or PSB sampling. Respiratory samples from expectoration must show $<10$ squamous epithelial cells and $>25$ polymorphonuclear neutrophils per $100 x$ field to be suitable.

- Blood culture positive for S. aureus (and no other apparent primary source of infection outside the lung)

- Pleural fluid aspirate or lung tissue culture positive for S. aureus (only if obtained as part of the subject's necessary clinical management or post-mortem)

5. P. aeruginosa ICU pneumonia in not mechanically ventilated patients See S.03.3 but replace S. aureus with P. aeruginosa.

6. $P$. aeruginosa ICU pneumonia in not mechanically ventilated patients See S.03.4 but replace S. aureus with P. aeruginosa. 


$$
\pi \pi
$$




\title{
Staphylococcus aureus colonization and the occurrence of ICU pneumonia; ASPIRE-ICU, a prospective international cohort study
}

\author{
Fleur P. Paling ${ }^{1}$ \\ Derek Hazard ${ }^{2}$ \\ Marc J.M. Bonten ${ }^{1,6}$ \\ Herman Goossens ${ }^{5}$ \\ Hasan S. Jafri ${ }^{3}$, \\ Surbhi Malhotra-Kumar ${ }^{5}$ \\ Frangiscos Sifakis ${ }^{3,4}$ \\ Susanne Weber ${ }^{2}$ \\ Jan A.J.W. Kluytmans ${ }^{1,7}$ \\ and the ASPIRE-ICU Study Team
}

Submitted

\section{CHAPTER}

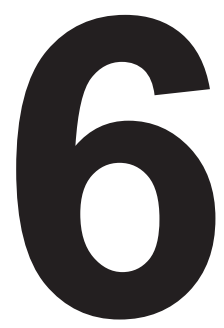

\footnotetext{
${ }^{1}$ Julius Center for Health Sciences and Primary Care, University Medical Center Utrecht, Utrecht University, Utrecht, Netherlands

${ }^{2}$ Institute of Medical Biometry and Statistics, University Medical Center Freiburg, Freiburg, Germany

${ }^{3}$ Microbial Sciences, R\&D BioPharmaceuticals, AstraZeneca, Gaithersburg, Maryland, United States of America

${ }^{4}$ Boehringer Ingelheim Pharmaceuticals, Inc, Ridgefield, CT, United States of America

${ }^{5}$ University of Antwerp, Antwerp, Belgium

${ }^{6}$ Department of Medical Microbiology, University Medical Center Utrecht, Utrecht University, Utrecht, Netherlands
} 


\section{ABSTRACT}

Importance: Carriage of Staphylococcus aureus (SA) is a risk factor for SA infection. Yet, associations between SA carriage and the development of SA Intensive Care Unit (ICU) Pneumonia (SAIP) have not been quantified accurately, and interpretation of available data is hampered because of variations in definitions.

Objective: To quantify associations between patient-related and contextual factors, including SA colonization status, and the occurrence of SAIP.

Design: Prospective, observational cohort study.

Setting: ICUs of 30 hospitals in eleven European countries, geographically spread in four regions.

Participants: In patients with an anticipated length of stay of $\geq 48$ hours and on mechanical ventilation (MV) at ICU admission, SA colonization was ascertained in the nose and lower respiratory tract. From this group SA colonized and non-colonized subjects were enrolled into the study cohort in a 1:1 ratio.

Main outcomes and measures: SAIP was defined as any pneumonia during ICU stay developing $\geq 48$ hours after ICU admission with SA isolated from lower respiratory tract or blood. The incidence of SAIP was derived in the study cohort and estimated upon weighted incidence calculation for the originating overarching population, while taking competing events into account. Weighted risk factor analysis was performed using Cox multivariate regression.

Results: The study cohort consisted of 1,933 patients, of whom 950 [49.1\%] were SA carrier at ICU admission. In all, 304 (15.7\%) developed ICU acquired pneumonia, of which 131 (6.8\%) had SAIP. Weighted SAIP incidences were 11.7 and 2.9 events per 1,000 patient days in ICU for SA colonized and non-colonized subjects, respectively (overall 4.9 per 1,000 patient days in ICU). The only independent risk factor for SAIP was SA colonization status at ICU admission (CSHR: 3.6, 95\% confidence interval: 2.2-6.0). There were marked regional differences in SAIP incidence and cause-specific hazard ratio (CSHR) for colonization status.

Conclusion and relevance: SAIP incidence was 4.9 per 1,000 ICU patient-days for patients on MV at ICU admission (or shortly thereafter). The daily risk of SAIP was 3.6 times higher in patients colonized with SA at ICU admission compared to patients without. 


\section{BACKGROUND}

Staphylococcus aureus (SA) both is a human commensal and an opportunistic pathogen. Healthy people carry the bacterium on the skin or in the respiratory tract, with a preference for the nose. Reported percentages of nasal carriage are around $25-30 \%{ }^{1,2}$. For healthy people, SA carriage is not a direct risk for infection ${ }^{3}$, but this changes in case of surgery or serious illness, for instance when treated in an intensive care unit (ICU). Although SA infections do occur in non-carriers, they occur far more frequently in those who are colonized with $S A^{4,5}$. Nosocomial pneumonia caused by SA frequently complicates hospitalization and may lead to severe consequences, especially when acquired in the $\mathrm{ICU}^{6,7}$.

Yet, little is known about the incidence of SA ICU pneumonia (SAIP) and about variations in incidence between countries and within countries, which partly results from differences in definitions and diagnostic detection methods used in previous studies ${ }^{5,8}$. Furthermore, for SAIP specifically, risk factors have not been quantified adequately. Apart from colonization status, other patient-related factors could increase the risk to develop SAIP. The ASPIRE-ICU (Advanced understanding of Staphylococcus aureus and Pseudomonas aeruginosa Infections in EuRopE - ICU) study was designed to quantify associations between patient-related and contextual factors, including SA colonization status at the time of ICU admission, and the occurrence of SAIP in eleven European countries ${ }^{9}$. 


\section{METHODS}

\section{Study Design, setting and participants}

ASPIRE-ICU was an observational, prospective cohort study among adult ICU patients at thirty hospitals in eleven European countries, recruiting subjects between June 2015 and October 2018. The study rationale and methods have been reported elsewhere ${ }^{9}$. For this study we identified ICUs with routine admission screening for SA carriage in nose and lower respiratory tract in patients with an expected LOS of $\geq 48 \mathrm{~h}$ and who were mechanically ventilated at ICU admission (or expected to be ventilated within $24 \mathrm{~h}$ ).

In summary, the study considered two populations; an overarching source population consisting of consecutive patients admitted to ICU with an expected length of stay of $\geq 48$ hours and mechanically ventilated at ICU admission (or expected to be ventilated within 24h), and the study cohort consisting of patients from the source population that provided consent for additional data and sample collection. Primary outcomes were derived from the study cohort. The source population was used to derive weighted incidence estimates using basic surveillance data and to determine differences between patients enrolled and not enrolled in the study cohort.

Patients in which both a nose and LRT screening sample could be obtained at ICU admission were eligible for the study cohort. LRT samples included endotracheal aspirate, spontaneously produced sputum or throat swabs if aspirates and sputum were not available. We aimed to enroll 2,000 study cohort subjects within 3 days after ICU admission, in a 1:1 ratio of SA colonized and non-colonized patients. Per ICU we enrolled all SA carriers, and approached the first eligible non-carrier after each enrolled SA carrier, in order to reach the predefined sample size with the pursued 1:1 ratio. Other in- and exclusion criteria and sample size calculations are described elsewhere ${ }^{9}$. Subjects with SA pneumonia at ICU admission were excluded from this analysis. During ICU stay study samples (e.g. endotracheal aspirates) were obtained three times weekly in the first week, two times weekly in the three weeks thereafter and at each day of protocol pneumonia (see elsewhere ${ }^{9}$ ). Criteria for establishing SAIP diagnosis were collected daily, as were the results from diagnostic tests taken during ICU stay for clinical reasons.

In each region in Europe, as described by the United Nations, we included at least one country ${ }^{10}$. A list of participating countries, including the final number of enrolled subjects per country can be found in the supplementary material (Table S1). The study protocol was approved by the institutional review boards or ethical review committees in each country and/or site. The study was registered as ClinicalTrials.gov: NCT02413242. 


\section{Study endpoints}

The primary outcome (incidence of SAIP through ICU stay) was assessed in several steps. First, protocol ICU pneumonia was based on daily assessment of four clinical criteria (the 'Daily pneumonia score', see Table 1). In case of one positive answer, a combination of objective major and minor criteria was assessed to categorize subjects as having protocol pneumonia or not (see elsewhere ${ }^{9}$ ). The primary endpoint SAIP was determined post-hoc based on isolation of SA from any LRT (including both clinical and study surveillance cultures) or blood culture in the three days before/after the day of pneumonia diagnosis. Secondary outcomes included all-cause ICU-acquired pneumonia and mortality at day 30 and day 90 after ICU admission.

Table 1. Criteria scored daily for each subject to assess protocol pneumonia diagnosis

\section{Daily pneumonia score}

\begin{tabular}{lc}
\hline - Any new antibiotic use in the last 24h & $\mathrm{Y} / \mathrm{N}$ \\
- Any new blood cultures drawn within the last 24h & $\mathrm{Y} / \mathrm{N}$ \\
- Any new chest X-ray (or CT) taken within the last 24h that show a new or worsening & $\mathrm{Y} / \mathrm{N}$ \\
& \\
infiltrate & $\mathrm{Y} / \mathrm{N}$ \\
\hline - Any other (new) reason occurred in the last 24h to suspect a pneumonia &
\end{tabular}

\section{Laboratory methods}

SA screening samples were processed locally on chromagar plates (Colorex ${ }^{\mathrm{TM}}$ staph aureus, Biotrading) using standardized methods. SA strains were selected on phenotypic criteria (pink or mauve color) and shipped to the central study laboratory. All predefined study samples were frozen (at - 80 degrees Celsius) and also shipped to the central lab. SA isolates from screening and clinical samples from patients with SAIP were compared using multilocus sequence typing (MLST).

\section{Statistical analysis}

Incidence calculation and primary endpoints

The incidence of SAIP was determined in the study cohort and estimated for the source population using weighting methods. The weighting methods used the observed proportion of SA carriage in the source population in combination with the likelihood of patients to be included as study subjects, stratified per country to calculate the incidence density estimate for the overall source population. These methods are described in more detail in supplementary file S1. Unweighted incidence calculations for the study subjects will be provided in the supplementary files. Incidence density is described by SA colonization status and region using a Cox survival analysis and taking into account the competing events death and ICU discharge without SAIP ${ }^{11,12}$. 


\section{Risk factor analysis}

Cause-specific hazards were determined for SAIP and the competing events, representing the daily 'risk' for a patient at a specific time to acquire each event. The next step was a weighted risk factor analysis for each competing event, yielding univariate causespecific hazard ratios (CSHRs) per exposure status. Because of anticipated differences between countries, the cause-specific Cox model was stratified per country. Lastly, a multivariate Cox regression survival analysis was performed, using variables selected from the univariate analysis to quantify the cumulative risk of acquiring SAIP in the presence of competing events. Statistical analyses were performed using $R$ version 3.6.1 ${ }^{13}$.

\section{Variable selection}

For univariate analysis, the following variables were selected prior to analysis, based on clinical reasoning and published data: SA colonization status, gender, body mass index (BMI), Acute Physiology, Age, Chronic Health Evaluation (APACHE) IV score, origin prior to ICU stay, prior antibiotic use (defined as any systemic antibiotic use for $\geq 1$ day within the 2 weeks prior to ICU stay), neurotrauma (admitted for trauma and Glasgow Coma Scale of $\leq 8$ ), pneumonia diagnosis, active SA infection (other than pneumonia), diabetes mellitus, bed head elevation during ICU stay, and peptic ulcer prophylaxis during ICU stay. Unless stated otherwise, all variables were measured at ICU admission. Variables such as age, chronic pulmonary disease, immunodeficiency status and mechanical ventilation were not included because of overlap with the APACHE IV score and/or inclusion criteria. Variables were selected for the multivariate model in case they yielded a p-value $<0.157$ (roughly corresponding to Akaike's information criterion) in any of the competing events' univariate analysis, abiding by the rule of thumb of 1 covariate per 10 events ${ }^{14,15}$. Missing data on risk factors were imputed using multiple imputation methods.

\section{Sensitivity analysis}

To determine the robustness of results we performed several sensitivity analyses, all on unweighted data. Firstly, we checked whether exclusion of 26 subjects from one site changed results, as contact with the site was lost at the end of subject recruitment and data could not be verified. Secondly, we determined to what extent excluding subjects with missing pneumonia information on at least two days and for $\geq 30 \%$ of days in total influenced results. Lastly, the complete analysis was repeated 11 times, each time excluding 1 country. 


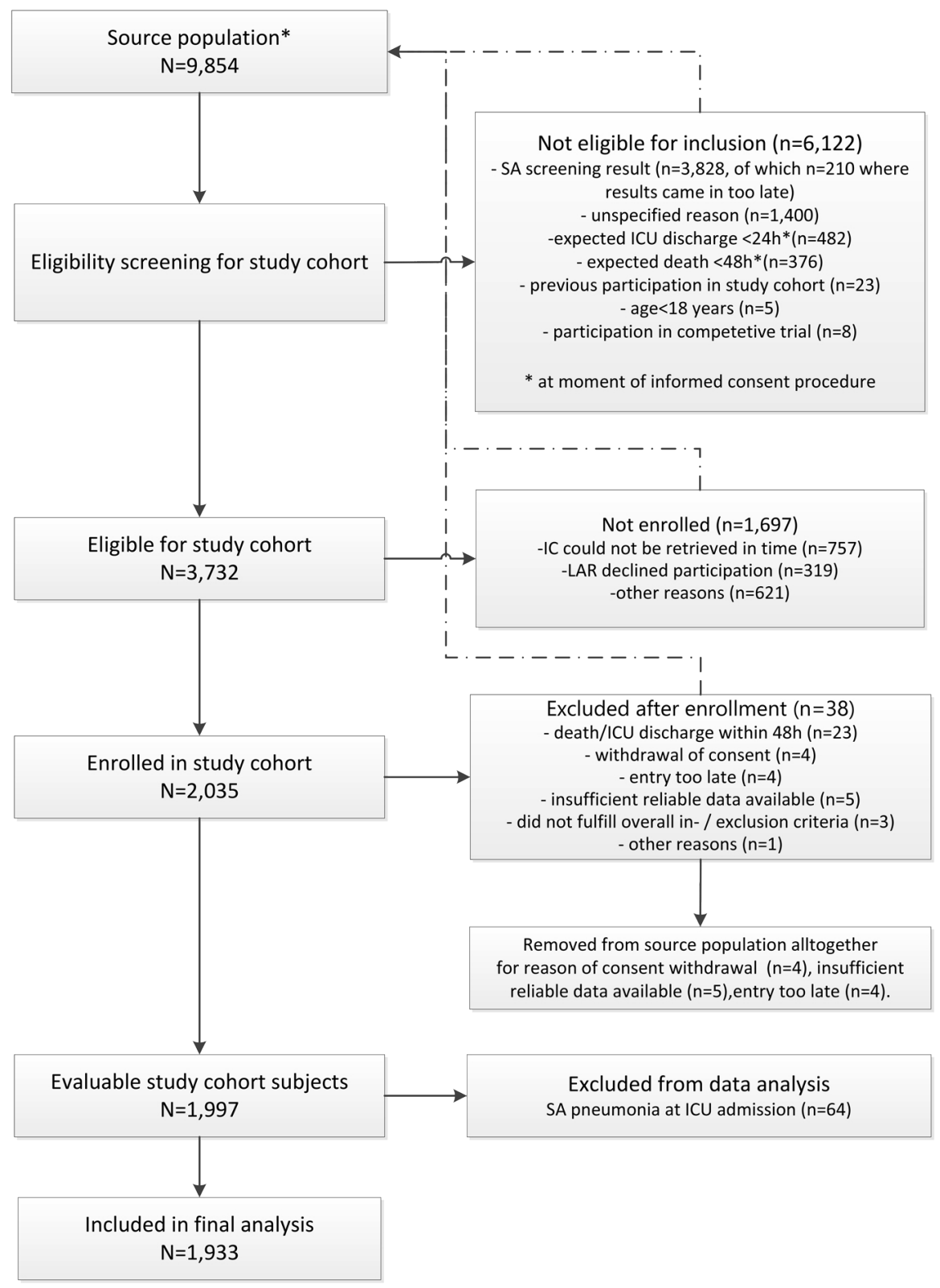

Figure 1. Flowchart of patients / subjects within ASPIRE-ICU

* Final evaluable number of patients in source population is 9,841 , because of the 13 subjects that were removed after enrollment in study cohort. 


\section{RESULTS}

\section{Patients}

In all, 9,841 patients were screened, of which 6,122 were considered ineligible for participation in the study cohort (Figure 1). Of those considered eligible ( $n=3,732), 2,035$ patients provided informed consent and were enrolled in the study cohort. Thirty-eight subjects were non-evaluable, mostly $(n=23)$ due to a length of stay (LOS) of $<48 \mathrm{~h}$. For the current analysis 64 subjects with SA pneumonia at ICU admission were excluded, resulting in a study cohort of 1,933 for this analysis.

\section{Source population and study cohort}

In the source population 2,440 patients (24.8\%) had SA colonization, 6,838 (69.5\%) had negative screening results and in 563 (5.7\%) colonization status could not be determined. In 445 patients, (4.5\%) either a nose or LRT sample was missing and SA colonization status was based on one available sample. Seventy patients were classified as SA carrier and 375 as non-carrier.

In the study cohort, 950 (49.1\%) of the 1,933 subjects were SA colonized at ICU admission. The average age of the subjects was 62.0 years and $64.8 \%$ were male (Table 2).

Most baseline characteristics were comparable between the source population and the study cohort, as were ICU mortality rates (see supplementary table S2). Study cohort subjects had slightly longer ICU stay (mean difference 1.3 days).

\section{Incidence of SAIP}

ICU-acquired pneumonia was observed in 304 subjects (15.7\%), 131 of which were categorized as SAIP, either based on local $(n=74)$ and/or central laboratory culture results ( $n=120)$; see supplementary Table S3). The weighted incidence estimate for SAIP in the original source population was 4.9 per 1000 days at risk. For SA colonized and non-colonized subjects, weighted incidences were 11.7 and 2.9 per 1000 days at risk, respectively. SAIP incidences differed between regions, as did associations between SA carriage and the occurrence of SAIP (Table 3-4). 
Table 2. Baseline characteristics.

\begin{tabular}{|c|c|c|c|c|}
\hline & & SA+ & SA- & Total \\
\hline & & \multicolumn{3}{|c|}{ as $\mathrm{n}(\%)$ or mean (SD) } \\
\hline Age & & $60,8(17.1)$ & $63,1(14.8)$ & $62,0(16.0)$ \\
\hline \multirow[t]{2}{*}{ Gender } & Male & $634(66.7)$ & $618(62.9)$ & $1252(64.8)$ \\
\hline & Female & $316(33.3)$ & $365(37.1)$ & $6801(35.2)$ \\
\hline $\begin{array}{l}\text { Origin prior to ICU } \\
\text { stay }\end{array}$ & $\begin{array}{l}\text { Home/ } \\
\text { community }\end{array}$ & $567(59.7)$ & 491 (49.9) & $1058(54.7)$ \\
\hline$n=5$ unknown & $\begin{array}{l}\text { Health care } \\
\text { related }\end{array}$ & $382(40.2)$ & $488(49.6)$ & $870(45.0)$ \\
\hline APACHE IV score ${ }^{a}$ & & $72,2(38.2)$ & $72,0(37.9)$ & $72,1(38.0)$ \\
\hline BMI & & $27,2(6.4)$ & $27,4(5.9)$ & $27,3(6.1)$ \\
\hline \multirow[t]{4}{*}{ Region } & North & $123(12.9)$ & $128(13.0)$ & $251(13.0)$ \\
\hline & South & $411(43.3)$ & $411(41.8)$ & $822(42.5)$ \\
\hline & East & $193(20.3)$ & $202(20.5)$ & 395 (20.4) \\
\hline & West & $223(23.5)$ & $242(24.6)$ & $465(24.1)$ \\
\hline \multirow[t]{4}{*}{ Admission specialty } & Medical & 484 (50.9) & $464(47.2)$ & $948(49.0)$ \\
\hline & Trauma & $204(21.5)$ & 169 (17.2) & 373 (19.3) \\
\hline & $\begin{array}{l}\text { Surgical } \\
\text { cardiothoracic }\end{array}$ & $49(5.2)$ & $74(7.5)$ & $123(6.4)$ \\
\hline & Surgical other & $213(22.4)$ & $276(28.1)$ & 489 (25.3) \\
\hline \multirow[t]{2}{*}{ Surgery ${ }^{b}$} & Emergency & $286(30.1)$ & $338(34.4)$ & $624(32.3)$ \\
\hline & Elective & $76(8.0)$ & $98(10.0)$ & $174(9.0)$ \\
\hline Neurotrauma $^{a}$ & & $120(12.6)$ & $86(8.7)$ & 206 (10.7) \\
\hline Prior antibiotic use & Yes & $179(18.8)$ & $293(29.8)$ & $472(24.4)$ \\
\hline \multirow[t]{2}{*}{$\mathrm{n}=187$ unknown } & No & $674(70.9)$ & $600(61.0)$ & 1274 (65.9) \\
\hline & Unknown & $97(10.2)$ & $92(9.2)$ & $187(9.7)$ \\
\hline $\begin{array}{l}\text { Diabetes mellitus } \\
\mathrm{n}=2 \text { missing }\end{array}$ & & $183(19.3)$ & $199(20,3)$ & $382(19.8)$ \\
\hline $\begin{array}{l}\text { Pneumonia }{ }^{a} \\
n=3 \text { unknown }\end{array}$ & & $142(14.9)$ & $184(18.7)$ & 326 (16.9) \\
\hline $\begin{array}{l}\text { Active } S A \text { infection }{ }^{\mathrm{a}, \mathrm{c}} \\
\mathrm{n}=4 \text { unknown }\end{array}$ & & 39 (4.1) & $15(1.5)$ & $54(2.8)$ \\
\hline Total & & 950 (100) & 983 (100) & $1933(100)$ \\
\hline
\end{tabular}

$\mathrm{SA}=\mathrm{S}$. aureus, $\mathrm{SD}=$ standard deviation, $\mathrm{ICU}=$ intensive care unit, $\mathrm{APACHE}=$ Acute Physiology, Age, Chronic Health Evaluation, $\mathrm{BMI}=$ body mass index.

${ }^{a}$ At ICU admission. ${ }^{b}$ In case a trauma patient needed surgery related to this trauma, this was assumed to be emergency surgery. ${ }^{\mathrm{C}}$ Other than pneumonia at ICU admission. 
The incidence of SAIP in SA carriers ranged from 17.6 in the northern region to 6.2 per 1000 days in the southern region. The median time to SAIP varied from 3 days for colonized patients in the western to 7.5 days in non-colonized patients in the southern region. Weighted cumulative incidence functions (CIFs) for SAIP per colonization status demonstrate that in SA carriers most SAIP episodes occurred in the first week of ICU admission (Figure 2a). The occurrence of SAIP in relation to the competing events (ICU discharge and death) is depicted in Figure $2 \mathrm{~b}$. Unweighted incidence data, incidence numbers stratified for sample type SA positivity and weighted CIFs per region are provided in the supplementary figures (Table S4-7, Figures SF1-SF6). The average number of microbiological cultures from respiratory samples and blood per subject that were locally obtained for clinical reasons varied between 0.29 to 0.74 per day over the different regions (Table S8).

\section{Colonizing vs. infecting strains}

Ninety-nine patients developed SAIP after prior SA colonization at ICU admission. Genetic comparison of SA isolates associated with colonization and infection within these individual patients was possible in 84 episodes, due to unavailability of either the infecting strain $(n=10)$ or the colonizing strain $(n=5)$ in the central laboratory. In $57(68 \%)$ of these 84 paired strains, MLST types were identical for the colonizing and infecting strains. Proportions of similarity ranged from 95\% (19 of 20 pairs) in the western region to $49 \%$ (16 of 33 pairs) in the southern region. The most dominant MLST types were ST239 ( $n=19$, of which $n=11$ in one region) for infecting and ST30 $(n=11)$ for colonizing strains.

\section{Risk factor analysis}

The univariate CSHR for developing SAIP for SA colonized compared to non-colonized subjects was 4.1 (95\% Cl 2.5-6.9, p<0.001). Pneumonia diagnosis at ICU admission (excluding those caused by SA) appeared protective for developing SAIP (CSHR: 0.4, $95 \% \mathrm{Cl} 0.2-0.9, \mathrm{p}=0.03$, Table 5). CSHRs for death and ICU discharge without SAIP can be found in the supplementary table S9. Based on the univariate analysis, eleven variables were included in the multivariate analysis, yielding a CSHR of $3.6(95 \% \mathrm{Cl} 2.2-$ $6.0, p<0.001)$ to develop SAIP for colonized patients compared to non-colonized patients (Table 5). Unweighted CSHRs (including for competing events) are in the supplementary tables S10-11. 
Table 3. Incidence of SA ICU pneumonia (weighted).

\begin{tabular}{llllll}
\hline & $\mathbf{n}$ & Days at risk & Risk N (\%) & $\begin{array}{l}\text { Rate N / 1000 } \\
\text { days at risk }\end{array}$ & $\begin{array}{l}\text { Time to SAIP } \\
\text { (median, in days) }\end{array}$ \\
\hline $\begin{array}{l}\text { Colonization status } \\
\text { - SA positive }\end{array}$ & 2,204 & 22,266 & $261(11.8 \%)$ & 11.7 & 4 \\
- SA negative & 7,221 & 79,711 & $234(3.2 \%)$ & 2.9 & 6 \\
Region & & & & & \\
- North & 1,894 & 22,161 & $162(8.8 \%)$ & 7.3 & 5 \\
- South & 2,585 & 33,832 & $114(6.3 \%)$ & 3.3 & 7 \\
- East & 1,057 & 11,090 & $56(6.1 \%)$ & 5.0 & 5.5 \\
- West & 3,889 & 34,889 & $163(7.1 \%)$ & 4.7 & 4 \\
\hline Overall & 9,425 & 101,977 & $495(5.3 \%)$ & 4.9 & 5 \\
\hline
\end{tabular}

$\mathrm{SA}=\mathrm{S}$. aureus, $\mathrm{ICU}=$ intensive care unit.

Table 4. Incidence of SA ICU pneumonia per colonization status* per region (weighted).

\begin{tabular}{llllll}
\hline & $\mathbf{n}$ & Days at risk & Risk N (\%) & $\begin{array}{l}\text { Rate N / 1000 } \\
\text { days at risk }\end{array}$ & $\begin{array}{l}\text { Time to SAIP } \\
\text { (median, in days) }\end{array}$ \\
\hline SA positive & & & & & \\
Region & & & & & \\
- North & 411 & 4,152 & $73(17.8 \%)$ & 17.6 & 4 \\
- South & 709 & 9,012 & $56(7.9 \%)$ & 6.2 & 6 \\
- East & 291 & 2,858 & $33(11.3 \%)$ & 11.5 & 5 \\
- West & 793 & 6,244 & $99(12.5 \%)$ & 15.9 & 3 \\
\hline Total (SA positive) & 2,204 & 22,266 & $261(11.8 \%)$ & 11.7 & 4 \\
\hline SA negative & & & & & \\
Region & & & & & 6 \\
- North & 1,483 & 18,009 & $89(6.0 \%)$ & 4.9 & 7.5 \\
- South & 1,876 & 24,820 & $58(3.1 \%)$ & 2.3 & 6 \\
- East & 766 & 8,232 & $23(3.0 \%)$ & 2.8 & 4 \\
- West & 3,096 & 28,650 & $64(2.1 \%)$ & 2.2 & 6 \\
\hline Total (SA negative) & 7,221 & 79,711 & $234(3.2 \%)$ & 2.9 & \\
\hline
\end{tabular}

$\mathrm{SA}=\mathrm{S}$. aureus, $\mathrm{ICU}=$ intensive care unit. ${ }^{*}$ measured at $\mathrm{ICU}$ admission 
a)

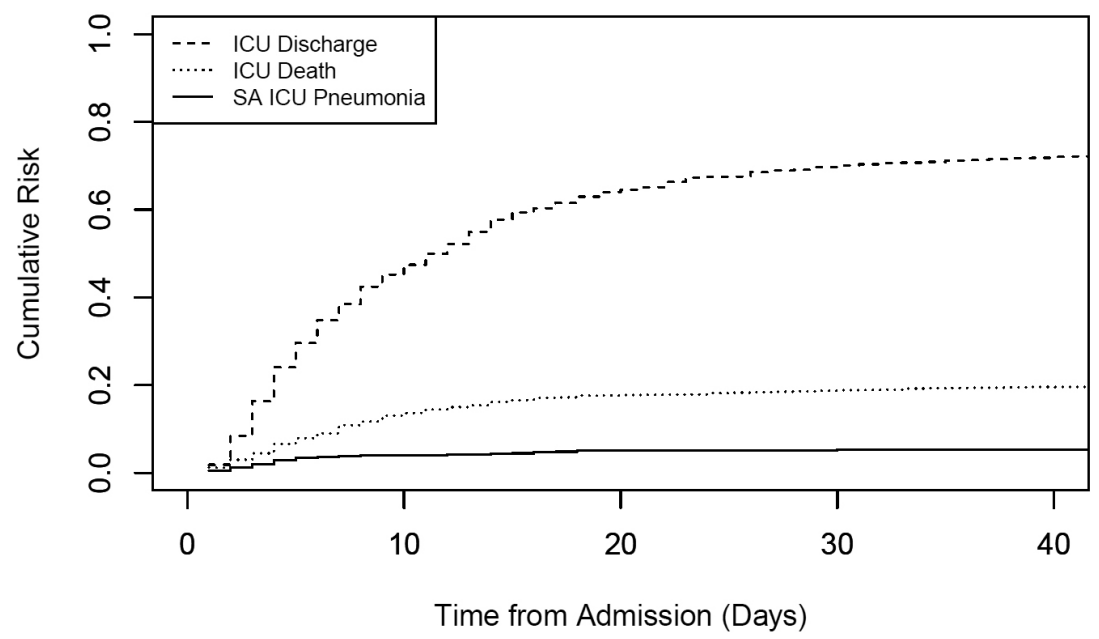

b)

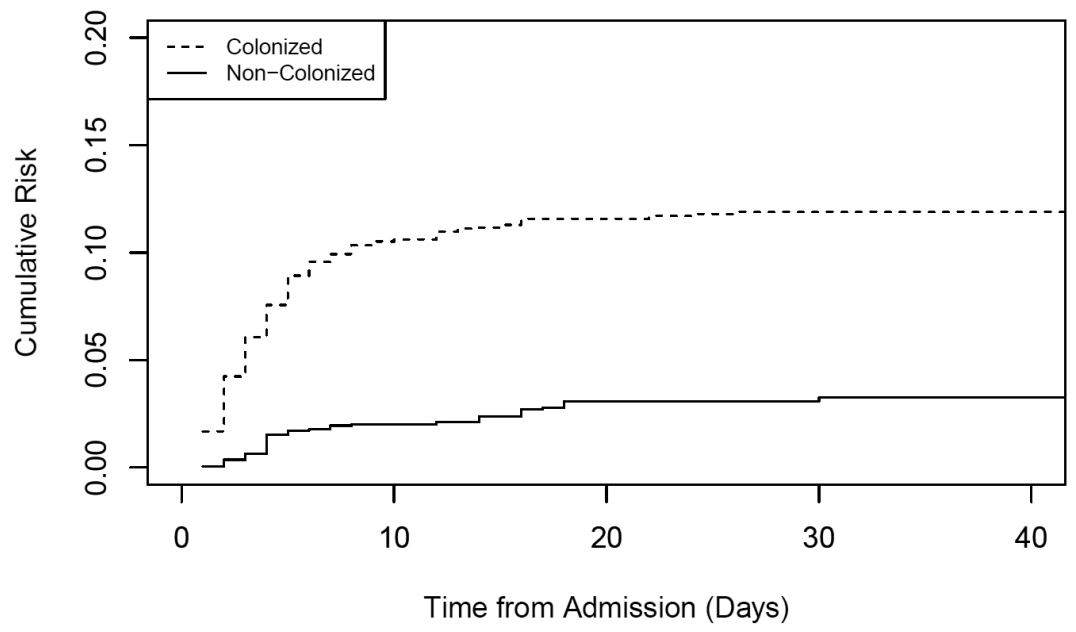

Figure 2. a) Cumulative incidence of SA ICU pneumonia and its competing events. b) Cumulative incidence function of SA ICU pneumonia per colonization status. 


\section{Sensitivity analyses}

Exclusion of the 26 subjects with unverified data did not change results. There were 172 (8.9\%) subjects with missing pneumonia information on at least two days; in 79 of these ( $4.1 \%$ of total) the amount exceeded $30 \%$ of the total amount expected. Exclusion of these subjects did not change the interpretation. Exclusion of one specific country from the analysis, changed the unweighted multivariate CSHR for SA colonization status from 3.1 (95\% Cl 2.1-4.7) to 5.0 (95\% $\mathrm{Cl} 3.0-8.5)$. Average changes in CSHR after removing other individual countries were 0.1 . No other clinically relevant of statistically significant estimate changes were observed in this sensitivity analysis.

Table 5. Risk factor analysis for SA ICU pneumonia.

\begin{tabular}{|c|c|c|c|c|}
\hline \multirow[t]{2}{*}{ Risk factor } & \multicolumn{2}{|c|}{ Univariate } & \multicolumn{2}{|c|}{ Multivariate } \\
\hline & CSHR $(95 \% \mathrm{Cl})$ & $\mathrm{p}$-value & $\operatorname{CSHR}(95 \% \mathrm{CI})$ & $\mathrm{p}$-value \\
\hline $\begin{array}{l}\text { SA colonization* } \\
\text { (non-colonized is reference } \\
\text { category) }\end{array}$ & $4.12(2.48-6.85)$ & $<0.001$ & $3.61(2.17-6.03)$ & $<0,001^{* *}$ \\
\hline $\begin{array}{l}\text { Male gender } \\
\text { (female is reference } \\
\text { category) }\end{array}$ & $0.89(0.51-1.56)$ & 0.69 & $\mathrm{NI}$ & \\
\hline $\begin{array}{l}\text { Health care setting origin } \\
\text { prior to ICU stay } \\
\text { (community is reference } \\
\text { category) }\end{array}$ & $0.73(0.42-1.28)$ & 0.27 & $0.94(0.44-2.00)$ & 0.87 \\
\hline APACHE IV score*† & $1.00(0.99-1.00)$ & 0.52 & $1.01(1.00-1.01)$ & 0.24 \\
\hline BMIt & $0.96(0.93-1.00)$ & 0.06 & $0.97(0.93-1.01)$ & 0.10 \\
\hline Neurotrauma* & 1.89 (1.00-3.53) & 0.05 & $1.23(0.65-2.30)$ & 0.53 \\
\hline Prior antibiotic use & $0.51(0.23-1.12)$ & 0.09 & $0.76(0.27-2.13)$ & 0.60 \\
\hline Diabetes mellitus & $0.93(0.47-1.83)$ & 0.83 & $1.14(0.56-2.32)$ & 0.73 \\
\hline Pneumonia* & $0.44(0.20-0.94)$ & 0.03 & $0.53(0.23-1.22)$ & 0.14 \\
\hline $\begin{array}{l}\text { Active SA infection other } \\
\text { than pneumonia * }\end{array}$ & $2.18(0.75-6.34)$ & 0.16 & $1.51(0.47-4.89)$ & 0.49 \\
\hline Peptic ulcer prophylaxis" & $1.85(0.66-5.17)$ & 0.24 & $1.72(0.61-4.79)$ & 0.52 \\
\hline Bed head elevation" & $0.66(0.15-2.90)$ & 0.58 & $1.00(0.22-4.66)$ & 1.00 \\
\hline
\end{tabular}

$\mathrm{CSHR}=$ cause specific hazard ratio, $\mathrm{Cl}=$ confidence interval, $\mathrm{SA}=\mathrm{S}$. aureus, $\mathrm{NI}=$ not included, $\mathrm{ICU}=$ intensive care unit, APACHE= Acute Physiology, Age, Chronic Health Evaluation, BMI= body mass index.

*At ICU admission. †Per point increase. \# During ICU stay. ** Significant in multivariate analysis. Variables that univariately were associated with $\mathrm{p}$-value $<0.157$ (bold) (for SAIP or competing events) were included in final multivariate model. 


\section{DISCUSSION}

In this prospective international study, patients colonized with SA the time of ICU admission had an almost four-fold higher risk of developing SAIP. Incidence densities for SAIP were 11.7 and 2.9 per 1,000 days at risk for SA-carriers and non-SA carriers, respectively, and 4.9 per 1,000 days at risk for the total ICU population. The incidence of SAIP and the strength of the association between carriage and SAIP differed between geographic European regions, as did microbiological culture frequency. SAIP incidence was highest in the northern and lowest in southern Europe, and SA carriage had the largest risk for SAIP in western Europe.

The observed regional differences in SAIP incidence and risks associated with SA colonization across Europe have not been reported earlier. Indeed, SAIP incidence may be influenced by differences in diagnostic work-up, which includes chest X-rays and microbiological cultures. The lowest SAIP incidence was observed in the region with the lowest culture frequency, and - vice versa - the second highest incidence in the region with the highest culture frequency. Yet, a scatterplot on the associations between culture frequency and SAIP incidence per study site suggests that culture frequency alone cannot explain these associations (Figure SF7). Unfortunately, the number of chest X-rays performed were not available.

Besides the differences in diagnostic strategies, regional differences in actual risk of SAIP related to colonization status may also result from differences in sources and transmission pathways of SA. We indeed actually observed a lower SAIP incidence among SA-colonized patients in a one region and/or a higher SAIP incidence in non-SAcolonized patients in another region (Table 4). In addition, the lowest risk associated with SA carriage on admission for developing SAIP was found in the in the region with the lowest genetic concordance between colonizing and infecting SA strains.

This suggests that cross-transmission of SA contributed more to SAIP in this region, than in regions with strong evidence for endogenous SA infection and with high heterogeneity in SA genotypes between patients with SAIP. This may have consequences for infection prevention. Targeted strategies interrupting progress from carriage to infection may be effective in settings where infections are predominantly from endogenous origin, whereas measures that reduce cross-transmission might be more effective in settings with indication of clonal transmission.

The association between SA colonization and infection has been demonstrated before ${ }^{4,5,16,17}$. The current study adds that there are regional differences in SAIP 
incidence, risk ratios between SA colonized and non-colonized patients to develop SAIP, and medical practice related to diagnostic culture frequencies. We consider the use of an objective definition for pneumonia, standardized laboratory screening methods and sophisticated statistical analyses as strengths of the current study. However, despite the use of objective criteria, pathogen-specific pneumonia diagnosis depends on diagnostic practices, which varied from country to country. The definition of SAIP as used in the current study is similar as definitions used in concurrent and upcoming intervention studies and was as such approved by the European Medicines Agency (EMA). Although, the definition as used included microbiological testing, it did not require quantitative measures, allowing pneumonia to be categorized as SAIP in case of low bacterial loads of SA or when other pathogens were also isolated. This may have caused misclassification and overestimation of the incidence of SAIP. Based on the current study we, therefore, question the validity of the diagnostic criteria as used for regulatory studies. With these definitions trials investigating preventive or therapeutic measures may be biased to zero, or in other words would demonstrate unjustified absence of treatment effects.

Another study limitation is the incompleteness of outcome data in some countries. However, the number of subjects in whom missing outcome data exceeded the predefined boundary was low ( $n=79,4.1 \%)$, and in $3(3.8 \%)$ of these subjects SAIP was observed, despite missing data. This may have led to a slight underestimation of the SAIP incidence in patients with prolonged LOS. A sensitivity analysis in which these patients were excluded yielded similar results.

In conclusion, in this study the overall incidence density of SAIP was 4.9 per 1,000 ICU days in patients on MV at ICU admission (or shortly thereafter). Specifically, SAIP incidence density was 11.7 and 2.9 for SA colonized and non-colonized subjects, respectively. SA colonization status was the only independent predictor for SAIP occurrence, with a CSHR of $3.6(95 \% \mathrm{CI} 2.2-6.0)$. Large regional differences in incidence rate as well as CSHR for colonization status were observed.

ASPIRE-ICU study team: Omar Ali, Martin Wolkewitz, Alexey Ruzin, Leen Timbermong, Christine Lammens, Sebastiaan Hullegie, Darren Troeman, Denise van Hout, Daniël Prins, Rubana Kalyani, Mark Eickhoff, Kathryn Shoemaker, Tuba Vilken, Jelle Vlaeminck, Jasmine Coppens, Thomas van der Schalk, Basil Britto Xavier, Atanas Temelkov, Nikolay Petrov, Rossitza Vatcheva, Michal Drab, Jaromir Vajter, Kadri Tamme, Muriel Fartoukh, Alain LePape, Mickael Landais, Gaetan Plantefève, Evelina Tacconelli, Achim Kaasch, Róbert Jurkinya, Dora Iványi, Miranda van Rijen, Olaf Cremer, Biljana Carevic, Jasna 
Jevdjić, Dolores Escudero, Miguel Sanchez Garcia, Cristina Prat-Aymerich, Borja Suberviola-Cañas, Angel Arenzana-Seisdedos Hürrem Bodur, Cenk Kirakli, Ilkay Bozkurt, David Pogson, Sandra Long, Matt Wise

Acknowledgements: We would like to thank Rianne Jahja, Jelle Lyskawa, Nicolette van der Sluis, Janet Couperus, Nienke Cuperus, Elien Burgers of the operational team of the University Medical Center of Utrecht; Liesbeth Bryssinck, Gert Leten, Anouk Vanderstraeten, Sofie Van Mieghem, Sabine Chapelle and Kitty Gys of the operational team of University of Antwerp, everybody of the operational teams of Seville and Limoges, all local investigators, monitors, other staff and participating patients.

Funding: This research project receives support from the Innovative Medicines Initiative Joint Undertaking under grant agreement no. 115523 and 115737 resources which are composed of financial contribution from the European Union Seventh Framework Programme (FP7/2007-2013) and EFPIA companies in kind contribution. 


\section{REFERENCES}

1. Kluytmans J, van Belkum A, Verbrugh H. Nasal carriage of Staphylococcus aureus: epidemiology, underlying mechanisms, and associated risks. Clin Microbiol Rev. 1997;10(3):505-520. http://www.pubmedcentral.nih.gov/articlerender. fcgi ?artid=172932\&tool=pmcentrez\&rendertype=abstract. Accessed September 8, 2014.

2. WILLIAMS RE. Healthy carriage of Staphylococcus aureus: its prevalence and importance. Bacteriol Rev. 1963;27:56-71. http://www.ncbi.nlm.nih.gov/pubmed/14000926. Accessed October 4, 2018.

3. Wertheim HFL, Melles DC, Vos MC, et al. The role of nasal carriage in Staphylococcus aureus infections. Lancet Infect Dis. 2005;5(12):751-762. doi:10.1016/S1473-3099(05)70295-4

4. von Eiff C, Becker K, Machka K, Stammer H, Peters G. Nasal carriage as a source of Staphylococcus aureus bacteremia. Study Group. N Engl J Med. 2001;344(1):11-16. doi:10.1056/NEJM200101043440102

5. Paling FP, Wolkewitz M, Bode LGM, et al. Staphylococcus aureus colonization at ICU admission as a risk factor for developing S. aureus ICU pneumonia. Clin Microbiol Infect. 2017;23(1). doi:10.1016/j.cmi.2016.09.022

6. Guzmán-Herrador B, Díaz Molina C, Allam MF, Fernández-Crehuet Navajas R. Underlying illness severity and outcome of nosocomial pneumonia: prospective cohort study in intensive care unit. J Hosp Infect. 2014;86(1):53-56. doi:10.1016/j.jhin.2013.09.014

7. Koulenti D, Tsigou E, Rello J. Nosocomial pneumonia in 27 ICUs in Europe: perspectives from the EU-VAP/CAP study. Eur J Clin Microbiol Infect Dis. 2017;36(11):1999-2006. doi:10.1007/ s10096-016-2703-z

8. Weiss E, Essaied W, Adrie C, Zahar J-R, Timsit J-F. Treatment of severe hospital-acquired and ventilator-associated pneumonia: a systematic review of inclusion and judgment criteria used in randomized controlled trials. Crit Care. 2017;21(1):162. doi:10.1186/s13054-017-1755-5

9. Paling FP, Troeman DPR, Wolkewitz M, et al. Rationale and design of ASPIRE-ICU: A prospective cohort study on the incidence and predictors of Staphylococcus aureus and Pseudomonas aeruginosa pneumonia in the ICU. BMC Infect Dis. 2017;17(1). doi:10.1186/s12879-017-2739-4

10. United Nations Statistics Division. Standard Country or Area Codes for Statistical Use.; 1999:54. https://unstats.un.org/unsd/methodology/m49/. Accessed October 29, 2019. 
11. Wolkewitz M, Cooper BS, Bonten MJM, Barnett AG, Schumacher M. Interpreting and comparing risks in the presence of competing events. BMJ. 2014;349:g5060. http://www. ncbi.nlm.nih.gov/pubmed/25146097. Accessed June 18, 2015.

12. Wolkewitz M, Vonberg RP, Grundmann H, et al. Risk factors for the development of nosocomial pneumonia and mortality on intensive care units: application of competing risks models. Crit Care. 2008;12(2):R44. doi:10.1186/cc6852

13. R Core Team. R: A Language and Environment for Statistical Computing. R Foundation for Statistical Computing. 2017. https://www.r-project.org.

14. Sauerbrei W. The Use of Resampling Methods to Simplify Regression Models in Medical Statistics. J R Stat Soc Ser C (Applied Stat. 1999;48(3):313-329. doi:10.1111/1467-9876.00155

15. Steyerberg EW. Clinical Prediction Models. Springer; 2009.

16. Corbella X, Domínguez MA, Pujol M, et al. Staphylococcus aureus nasal carriage as a marker for subsequent staphylococcal infections in intensive care unit patients. Eur J Clin Microbiol Infect Dis. 1997;16(5):351-357. http://www.ncbi.nlm.nih.gov/pubmed/9228474. Accessed September 8, 2014.

17. Pujol M, Peña C, Pallares R, et al. Nosocomial Staphylococcus aureus bacteremia among nasal carriers of methicillin-resistant and methicillin-susceptible strains. Am J Med. 1996;100(5):509-516. http://www.ncbi.nlm.nih.gov/pubmed/8644762. Accessed April 10, 2014. 


\section{SUPPLEMENTARY MATERIAL}

Supplementary Table S1: Participating countries and total included subjects per country

Supplementary Table S2: Baseline characteristics of participants vs. complete source population, stratified for SA colonization status.

Supplementary Table S3: SAIP categorization based on local and study culture results.

Supplementary Table S4: Incidence of SAIP (unweighted).

Supplementary Table S5: Incidence of SAIP per colonization status per region* (unweighted).

Supplementary Table S6: Incidence of SAIP per colonization status per stratified for sample type* (weighted).

Supplementary Table S7: Incidence of SAIP per colonization status per stratified for sample type (unweighted).

Supplementary Table S8: Average number of reported cultures and missing DPS (per subject).

Supplementary Table S9: Risk factor analysis (univariate, weighted) for competing events.

Supplementary Table S10: Risk factor analysis (univariate \& multivariate, unweighted) for SAIP.

Supplementary Table S11: Risk factor analysis (univariate, unweighted) for competing events.

Supplementary Figure SF1: Cumulative incidence function (CIF) for SAIP and competing events (unweighted).

Supplementary Figure SF2: CIF for SAIP per colonization status (unweighted).

Supplementary Figure SF3: CIF for SAIP per region (weighted).

Supplementary Figure SF4: CIF for SAIP per region (unweighted).

Supplementary Figure SF5: CIF for SAIP per colonization status per region (weighted).

Supplementary Figure SF6: CIF for SAIP per colonization status per region (unweighted).

Supplementary Figure SF7: SAIP incidence versus average number of cultures per subject per day over the 30 sites.

Supplementary File S1: Weighting methods. 
Table S1. Participating countries and total included subjects per country

\begin{tabular}{|c|c|c|c|}
\hline \multirow[t]{2}{*}{ Region } & \multirow[t]{2}{*}{ Country } & \multicolumn{2}{|l|}{ Participants } \\
\hline & & This analysis & All \\
\hline \multirow[t]{2}{*}{ North } & Estonia & 145 & 151 \\
\hline & United Kingdom & 106 & 106 \\
\hline \multirow[t]{3}{*}{ South } & Turkey & 157 & 158 \\
\hline & Serbia & 336 & 340 \\
\hline & Spain & 329 & 338 \\
\hline \multirow[t]{3}{*}{ East } & Bulgaria & 126 & 127 \\
\hline & Czech Republic & 196 & 205 \\
\hline & Hungary & 73 & 84 \\
\hline \multirow[t]{3}{*}{ West } & The Netherlands & 328 & 344 \\
\hline & France & 91 & 95 \\
\hline & Germany & 46 & 49 \\
\hline Total & & 1,933 & 1,997 \\
\hline
\end{tabular}




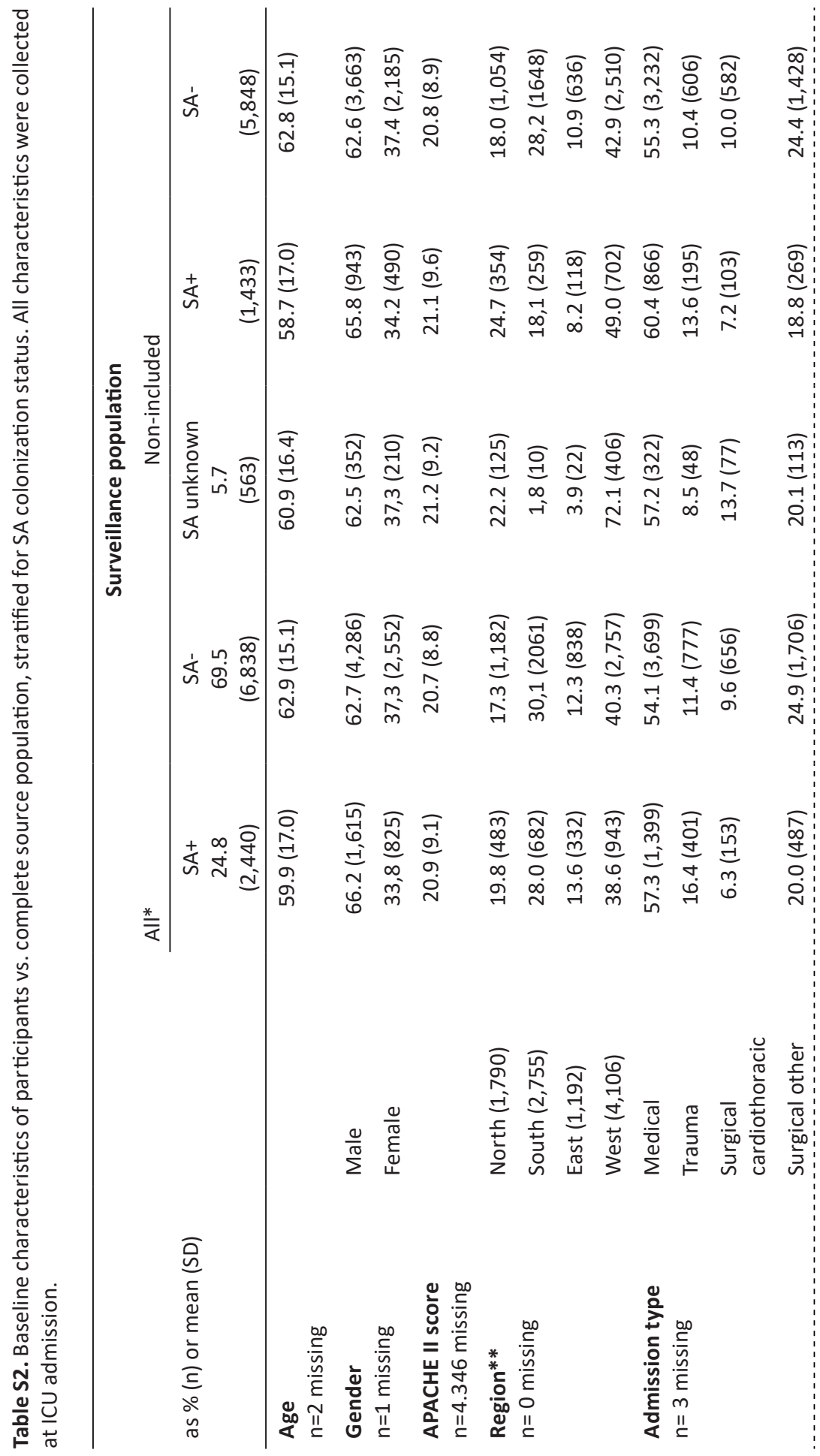




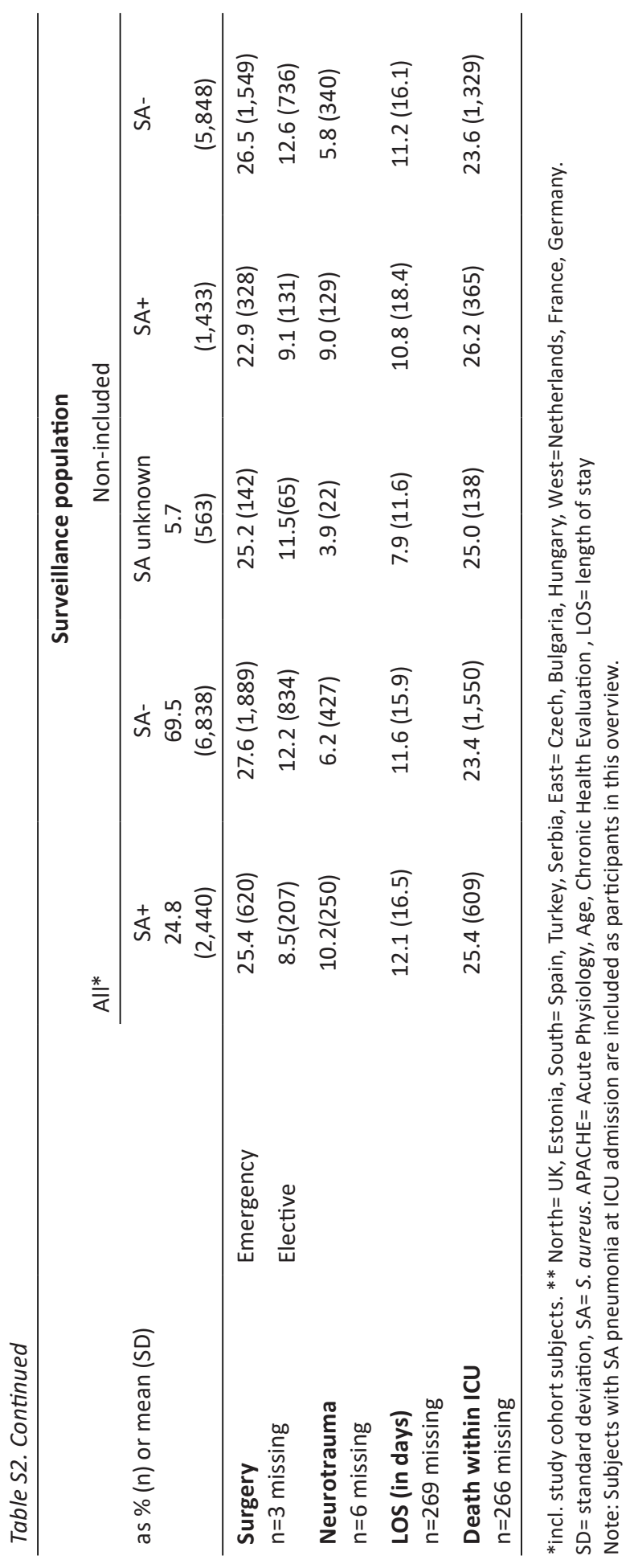


Table S3. SA ICU pneumonia categorization based on local and study culture results.

\begin{tabular}{lllll}
\hline & & \multicolumn{2}{l}{ Based on local cultures } & Total \\
& & Yes & No & \\
\hline Based on study cultures & Yes & 63 & 57 & 120 \\
& No & 9 & 162 & 171 \\
& N/A & 2 & 11 & \\
\hline Total & & 74 & 230 & 304 \\
\hline
\end{tabular}

$\mathrm{N} / \mathrm{A}=$ no study sample available centrally.

Table S4. Incidence of SA ICU pneumonia (unweighted).

\begin{tabular}{lllll}
\hline & Days at risk & Risk N (\%) & $\begin{array}{l}\text { Rate N / 1000 } \\
\text { days at risk }\end{array}$ & $\begin{array}{l}\text { Time to SAIP } \\
\text { (median, in days) }\end{array}$ \\
\hline $\begin{array}{l}\text { Colonization status } \\
\text { - SA positive }\end{array}$ & 10,052 & $99(10.4 \%)$ & 9.8 & 5 \\
- SA negative & 11,409 & $32(3.3 \%)$ & 2.8 & 6 \\
Region & & & & \\
- North & 2,602 & $22(8.8 \%)$ & 8.5 & 5 \\
- South & 10,864 & $52(6.3 \%)$ & 4.8 & 6.5 \\
- East & 3,905 & $24(6.1 \%)$ & 6.1 & 5.5 \\
- West & 4,090 & $33(7.1 \%)$ & 8.1 & 3 \\
\hline Overall & 21,461 & $131(6.8 \%)$ & 6.1 & 5 \\
\hline
\end{tabular}

$\mathrm{SA}=\mathrm{S}$. aureus, $\mathrm{ICU}=$ intensive care unit. 
Table S5. Incidence of SA ICU pneumonia per colonization status per region* (unweighted).

\begin{tabular}{lllll}
\hline & Days at risk & Risk N (\%) & $\begin{array}{l}\text { Rate N / 1000 } \\
\text { days at risk }\end{array}$ & $\begin{array}{l}\text { Time to SAIP } \\
\text { (median, in days) }\end{array}$ \\
\hline $\begin{array}{l}\text { SA positive } \\
\text { Region }\end{array}$ & & & \\
- North & 1,218 & $17(13.8 \%)$ & 14.0 & 4 \\
- South & 5,257 & $34(8.3 \%)$ & 6.5 & 6.5 \\
- East & 1,771 & $19(9.8 \%)$ & 9.8 & 5 \\
- West & 1,806 & $29(13.0 \%)$ & 13.0 & 3 \\
\hline Total (SA positive) & 10,052 & $99(10.4 \%)$ & 9.8 & 5 \\
\hline SA negative & & & & \\
Region & & & & 6 \\
- North & 1,384 & $5(3.9 \%)$ & 3.6 & 6.5 \\
- South & 5,607 & $18(4.4 \%)$ & 3.2 & 9.5 \\
- East & 2,134 & $5(2.5 \%)$ & 2.3 & 6 \\
- West & 2,284 & $4(1.7 \%)$ & 1.8 & \\
\hline Total (SA negative) & 11,409 & $32(3.3 \%)$ & 2.8 & \\
\hline
\end{tabular}

$\mathrm{SA}=S$. aureus, ICU= intensive care unit. * measured at ICU admission

Table S6. Incidence of SA ICU pneumonia per colonization status per stratified for sample type* (weighted).

\begin{tabular}{llllll}
\hline & $\mathbf{n}$ & Days at risk & Risk N (\%) & $\begin{array}{l}\text { Rate N / 1000 } \\
\text { days at risk }\end{array}$ & $\begin{array}{l}\text { Time to SAIP } \\
\text { (median, in days) }\end{array}$ \\
\hline Nose & & & & & \\
SA + & 1,867 & 19,146 & $209(11.2)$ & 10.9 & 5 \\
SA - & 7,551 & 82,814 & $283(3.7)$ & 3.4 & 5 \\
ETA & & & & & \\
SA + & 822 & 7,400 & $170(20.7)$ & 23.0 & 4 \\
SA - & 6,409 & 74,342 & $240(3.7)$ & 3.2 & 6 \\
Throat & & & & & \\
SA + & 323 & 3,277 & $29(9.0)$ & 8.8 & 2 \\
SA - & 2399 & 21,774 & $72(3.0)$ & 3.3 & 15 \\
Sputum & & & & & \\
SA + & 34 & 193 & $4(11.8)$ & 20.7 & 2 \\
SA - & 280 & 2,194 & $4(1.4)$ & 1.8 & 3.5 \\
\hline Overall & 9,425 & 101,977 & $495(5.3)$ & 4.9 & 4 \\
\hline
\end{tabular}

$\mathrm{SA}=\mathrm{S}$. aureus, ICU= intensive care unit, $\mathrm{SAIP}=\mathrm{SA} I \mathrm{CU}$ pneumonia, $\mathrm{ETA}=$ endotracheal aspirate, *measured at ICU admission 
Table S7. Incidence of SA ICU pneumonia per colonization status per stratified for sample type* (unweighted).

\begin{tabular}{llclll}
\hline & $\mathbf{n}$ & Days at risk & Risk N (\%) & $\begin{array}{l}\text { Rate N / 1000 } \\
\text { days at risk }\end{array}$ & $\begin{array}{l}\text { Time to SAIP } \\
\text { (median, in days) }\end{array}$ \\
\hline Nose & & & & & \\
SA + & 809 & 8,706 & $80(9.9)$ & 9.2 & 5 \\
SA - & 1,122 & 12,750 & $50(4.45)$ & 3.9 & 6 \\
ETA & & & & & \\
SA + & 356 & 3,373 & $61(16.9)$ & 18.1 & 4 \\
SA - & 1,240 & 14,871 & $58(4.7)$ & 3.9 & 6 \\
Throat & & & & & \\
SA + & 127 & 1,345 & $10(7.9)$ & 7.4 & 3.5 \\
SA - & 362 & 3,343 & $11(3.0)$ & 3.3 & 5 \\
Sputum & & & & & \\
SA + & 16 & 72 & $1(6.3)$ & 13.9 & 2 \\
SA - & 52 & 411 & $2(3.8)$ & 4.9 & 3.5 \\
\hline Overall & 1,933 & 21,461 & $131(6.8)$ & 6.1 & 5 \\
\hline
\end{tabular}

$\mathrm{SA}=\mathrm{S}$. aureus, ICU= intensive care unit, $\mathrm{SAIP}=\mathrm{SA} I \mathrm{ICU}$ pneumonia, ETA=endotracheal aspirate, *measured at ICU admission

Table S8. Average number of reported cultures and missing DPS (per subject).

\begin{tabular}{lllll}
\hline & $\begin{array}{l}\text { Local cultures / day } \\
(\mathbf{n})\end{array}$ & $\begin{array}{l}\text { Available sample centrally } \\
(\%)\end{array}$ & $\begin{array}{l}\text { Missing DPS } \\
(\mathbf{n})\end{array}$ & $\begin{array}{l}\text { Missing DPS } \\
(\%)\end{array}$ \\
\hline North & 0.34 & 95.2 & 0.52 & 7.7 \\
South & 0.29 & 90.7 & 0.48 & 5.8 \\
East & 0.43 & 93.6 & 0.92 & 9.0 \\
West & 0.74 & 77.8 & 0.33 & 9.2 \\
\hline All & 0.43 & 87.2 & 0.54 & 7.5 \\
\hline
\end{tabular}

DPS= daily pneumonia score 


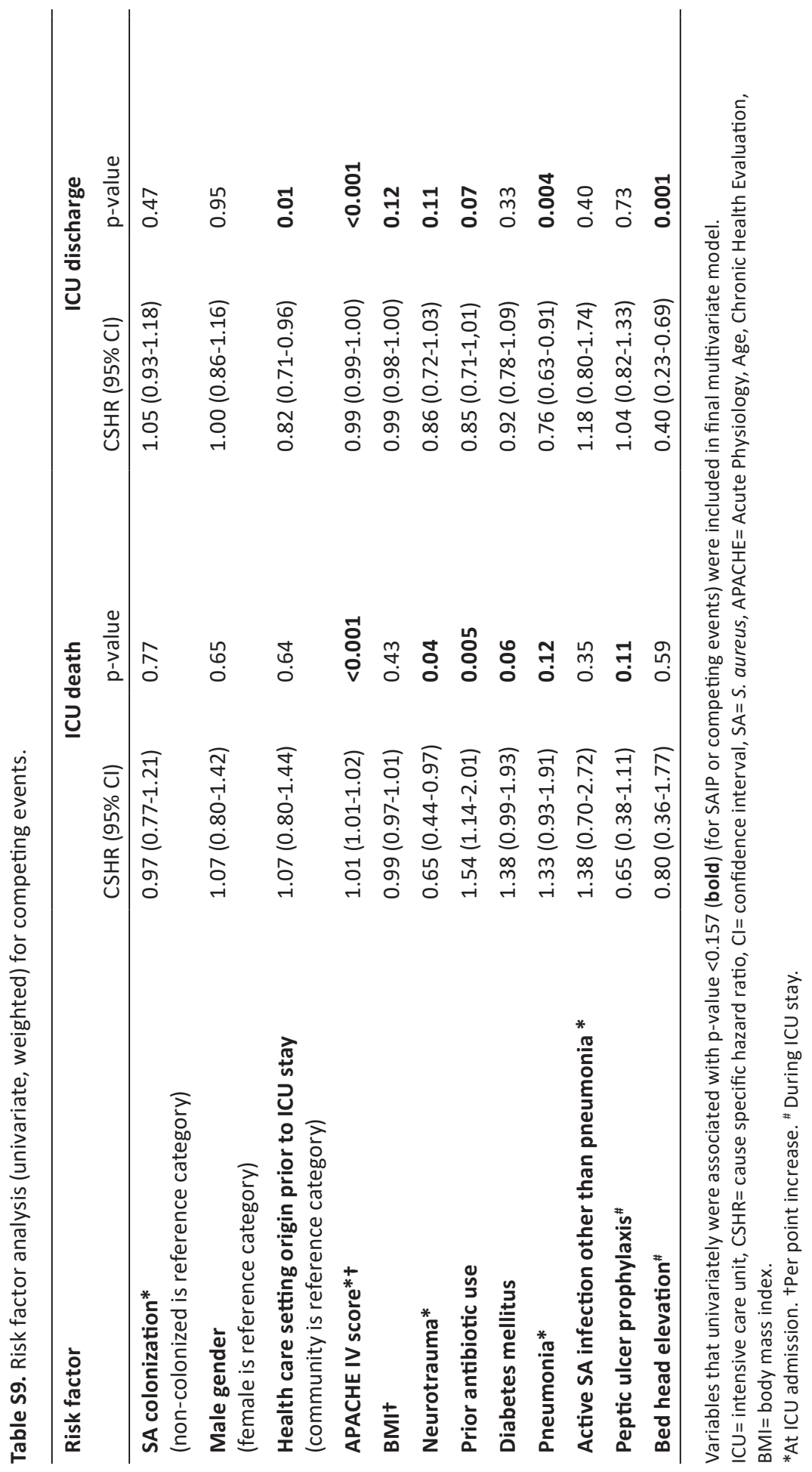




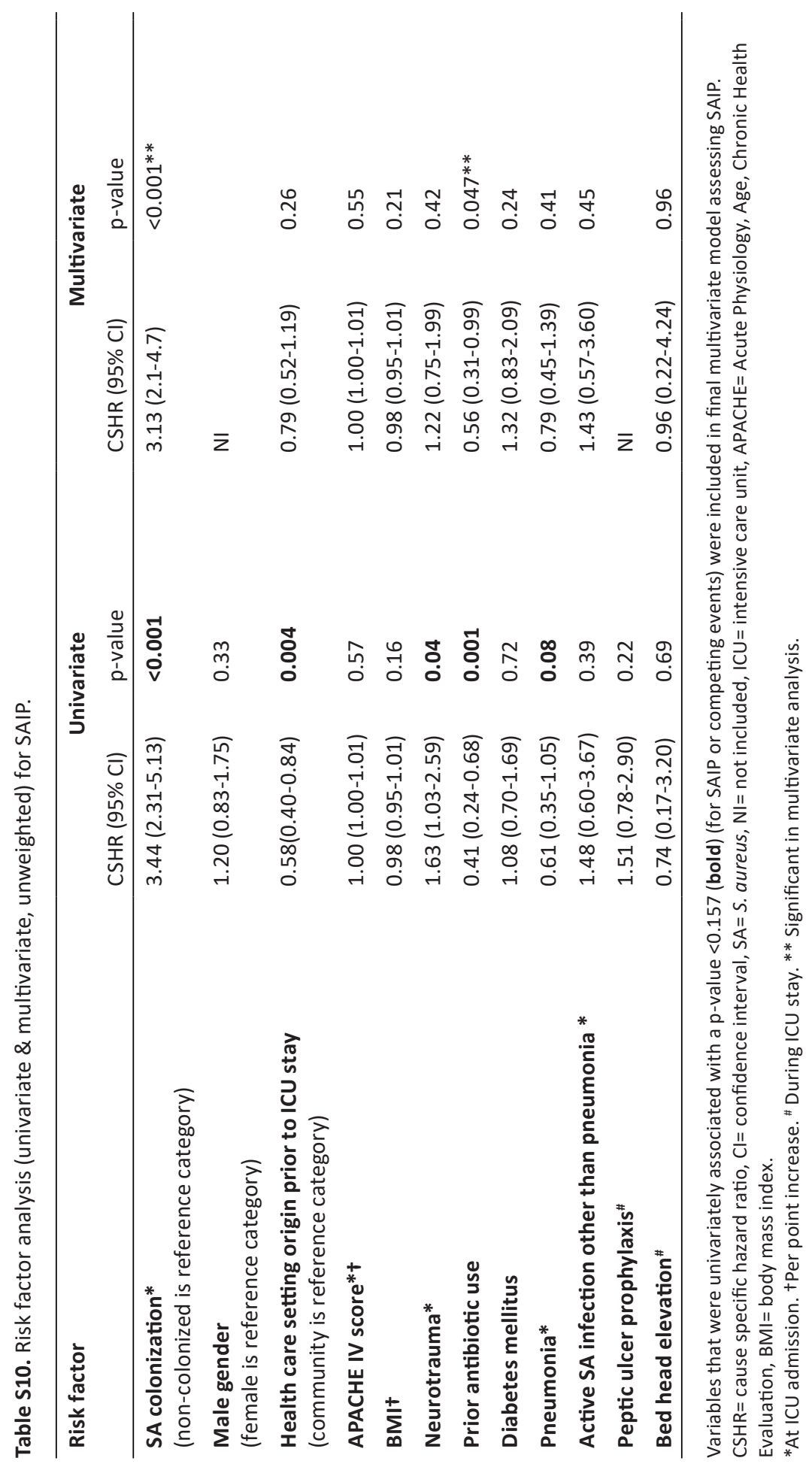




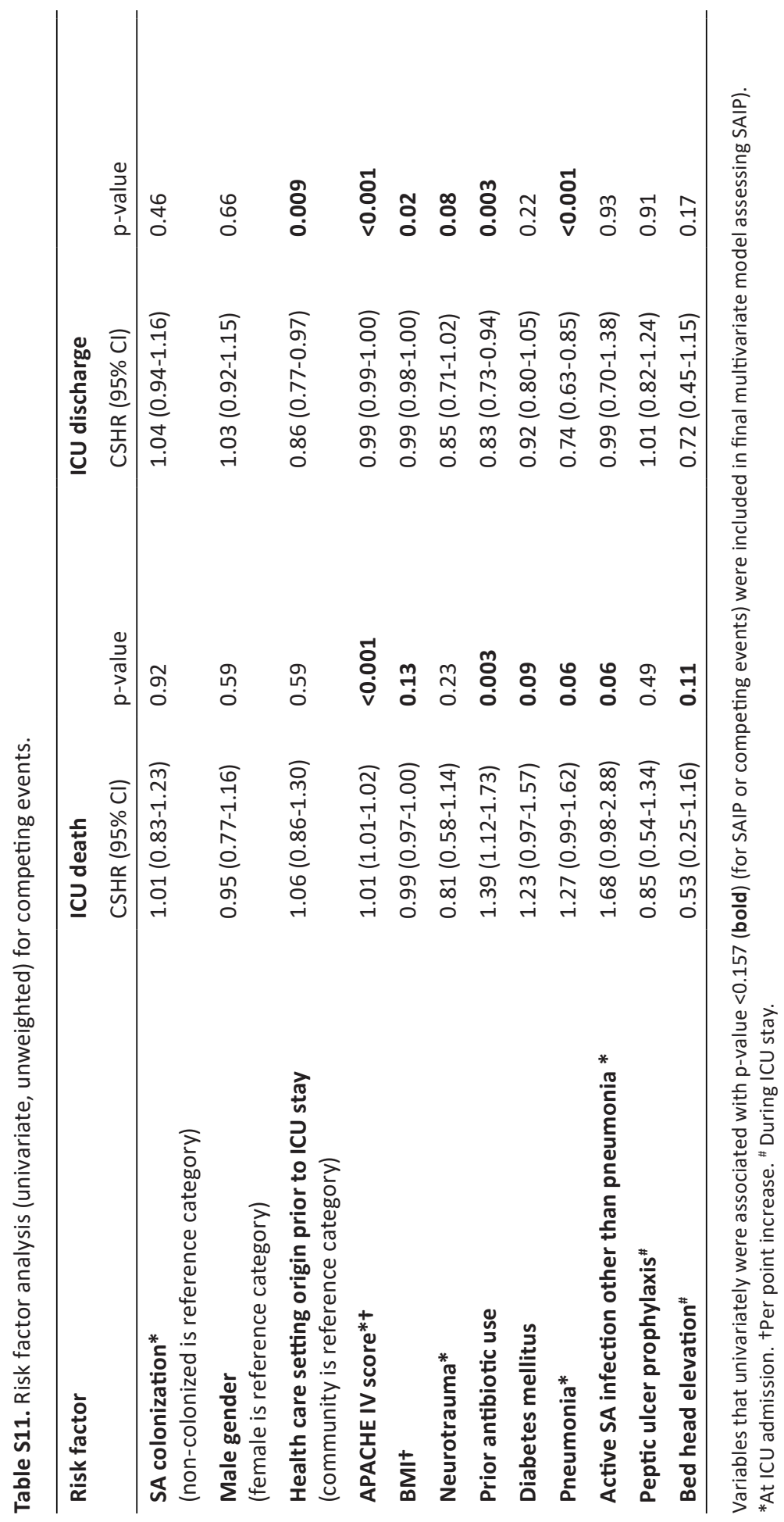




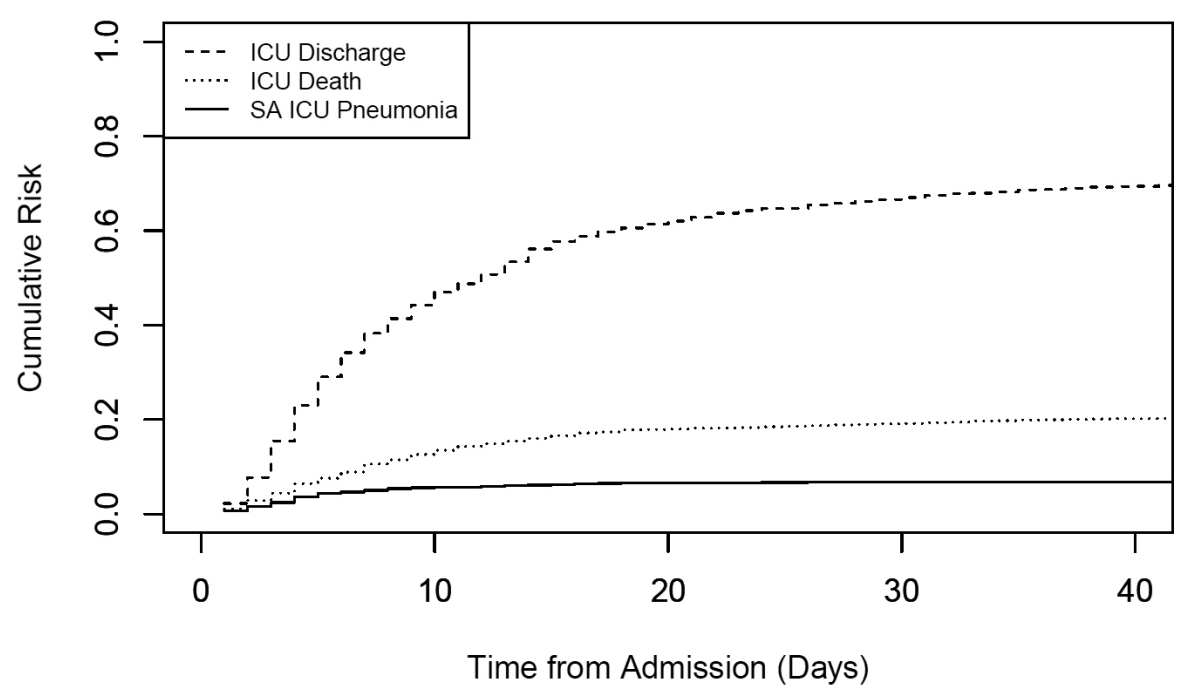

Supplementary Figure SF1: Cumulative incidence function (CIF) for SAIP and competing events (unweighted)

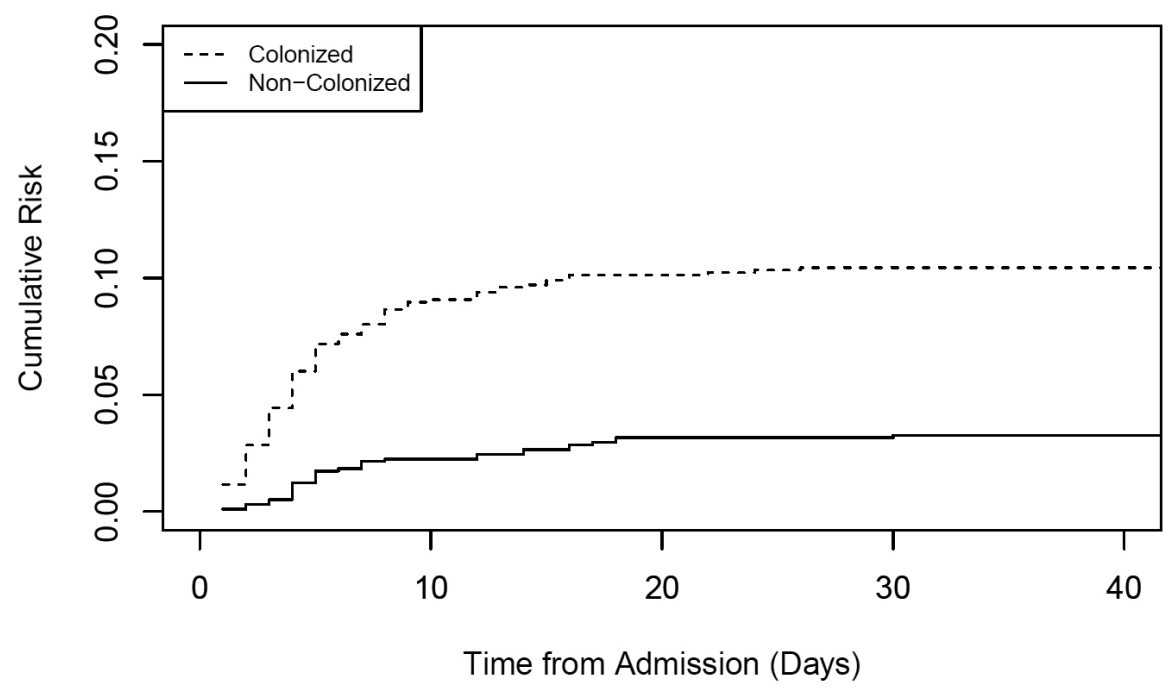

Supplementary Figure SF2: CIF for SAIP per colonization status (unweighted) 


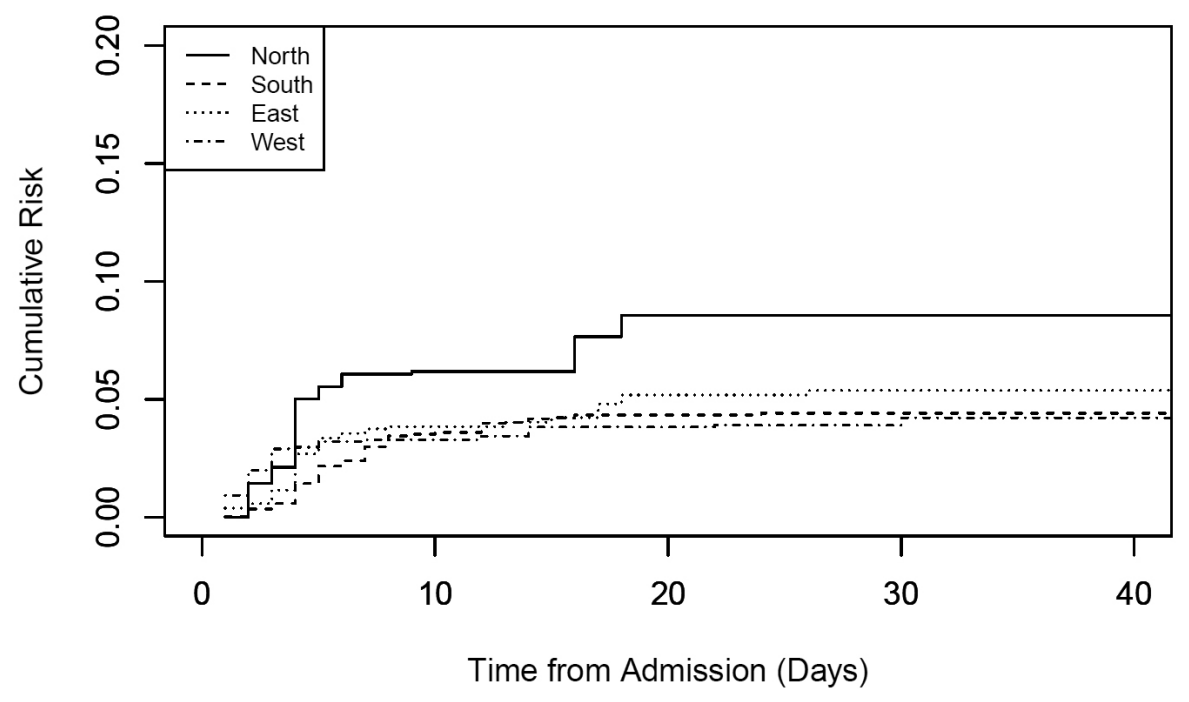

Supplementary Figure SF3: CIF for SAIP per region (weighted)

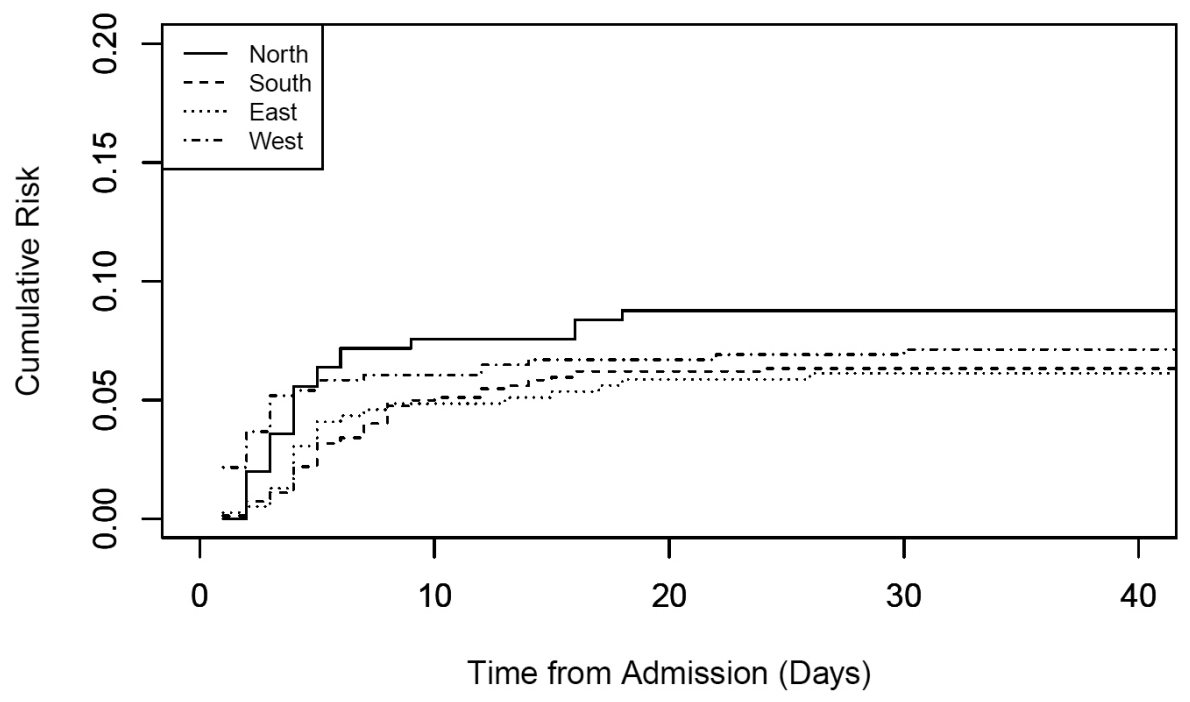

Supplementary Figure SF4: CIF for SAIP per region (unweighted) 


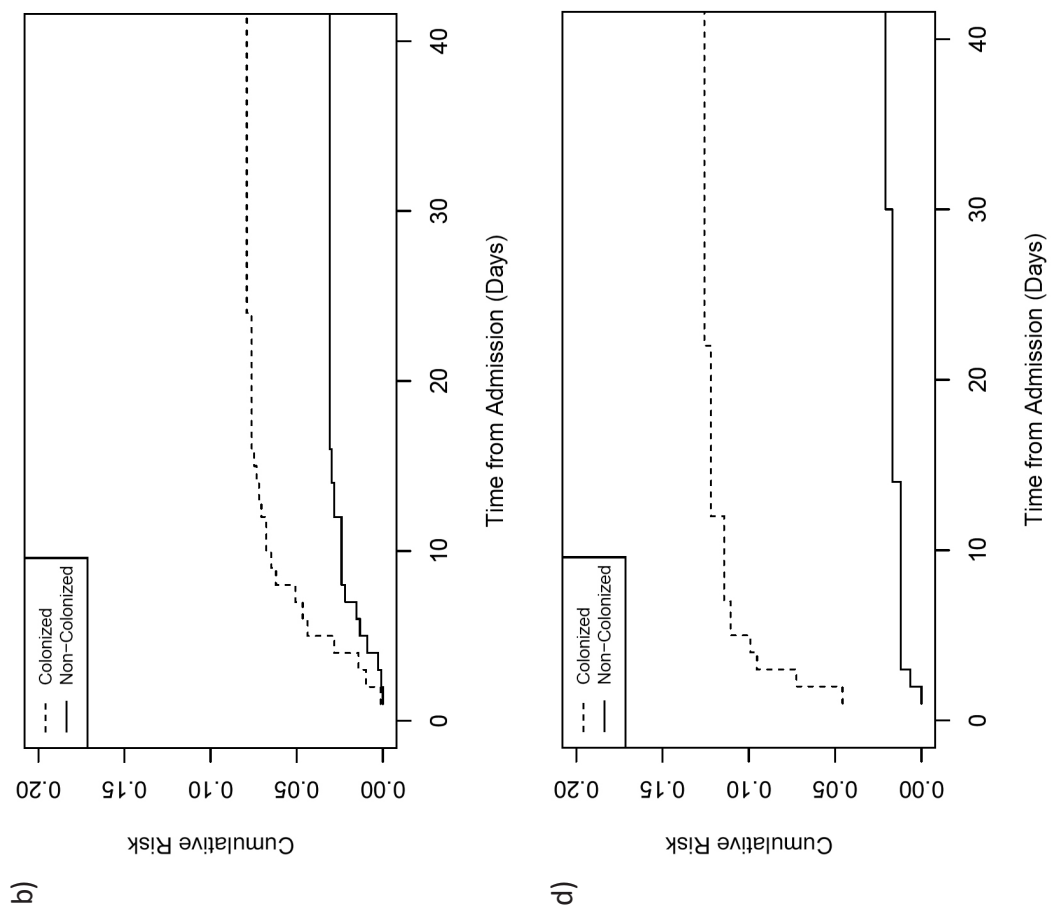

$\frac{\dot{\bar{d}}}{\stackrel{ \pm}{\frac{1}{00}}}$
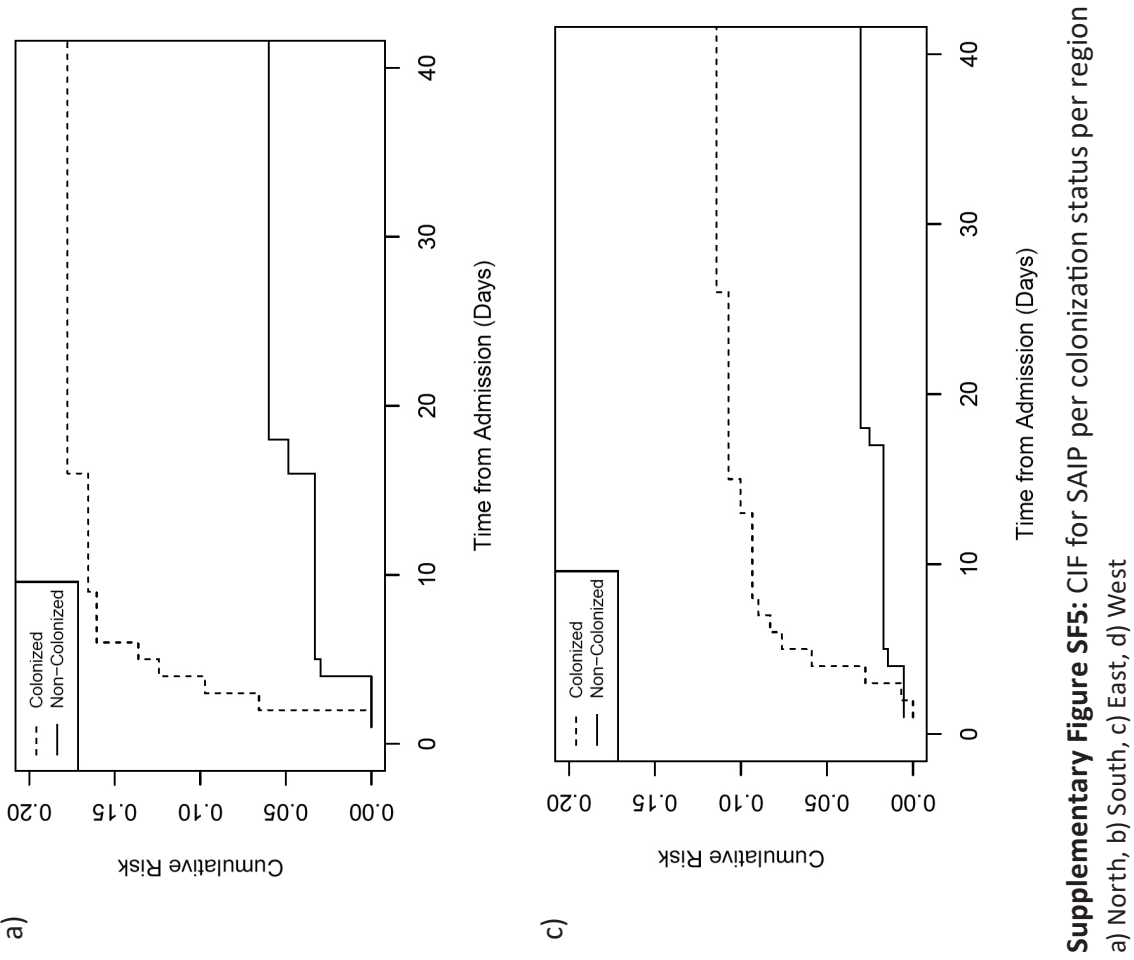

$\bar{\sigma}$ 

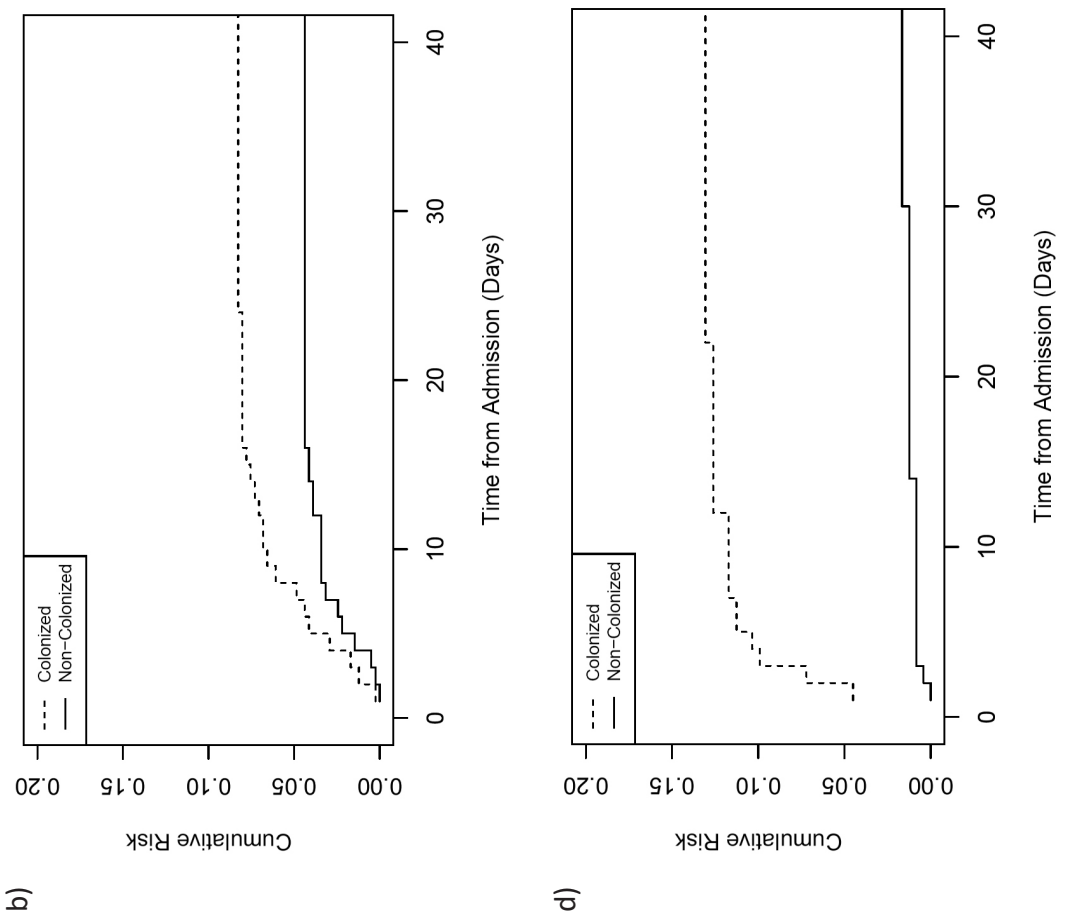

อ
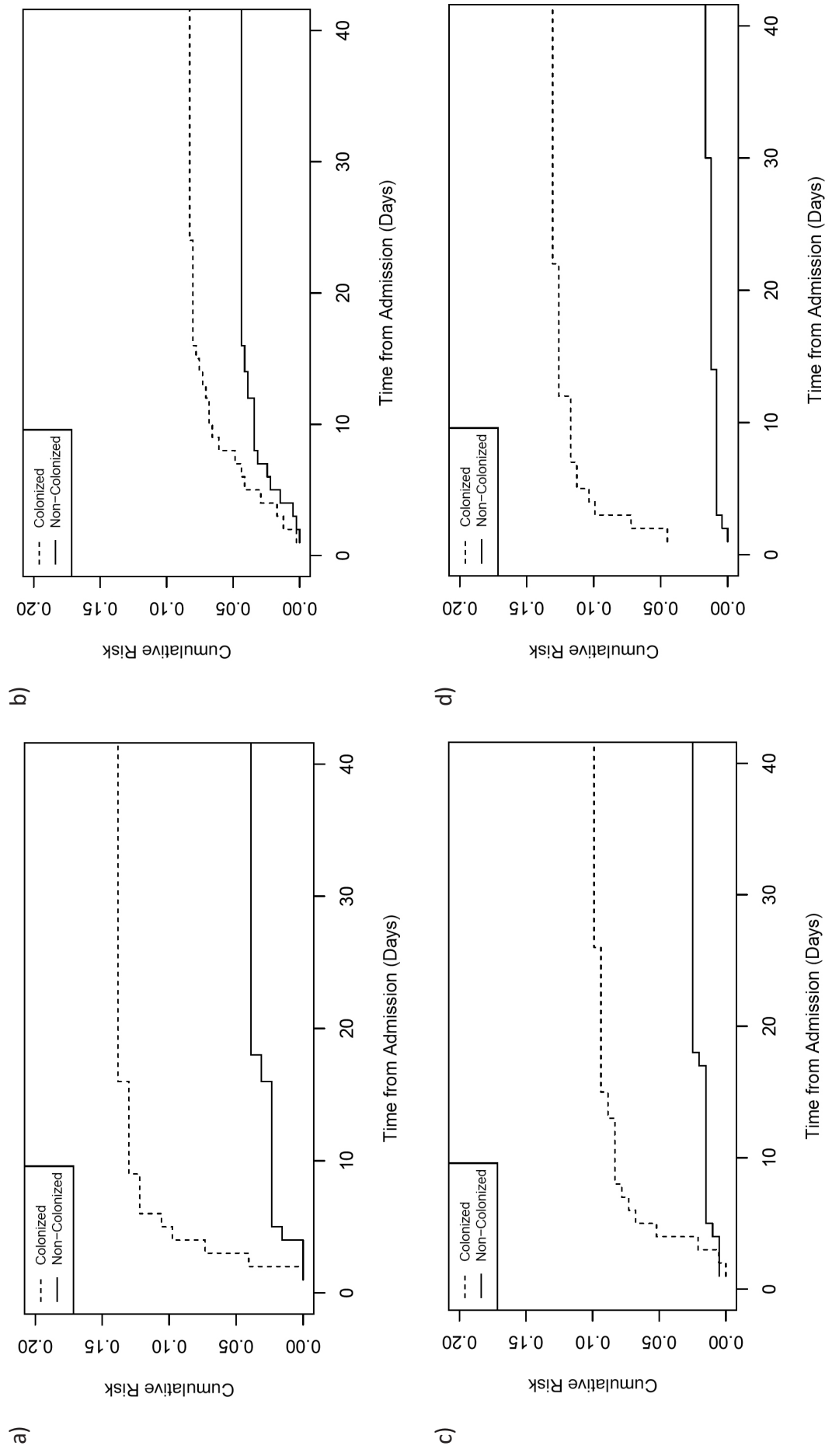

6

$\bar{\pi}$

$\overline{0}$

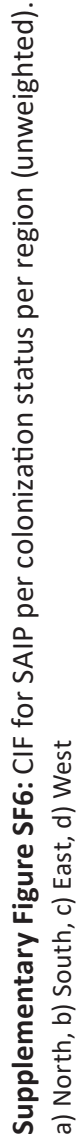




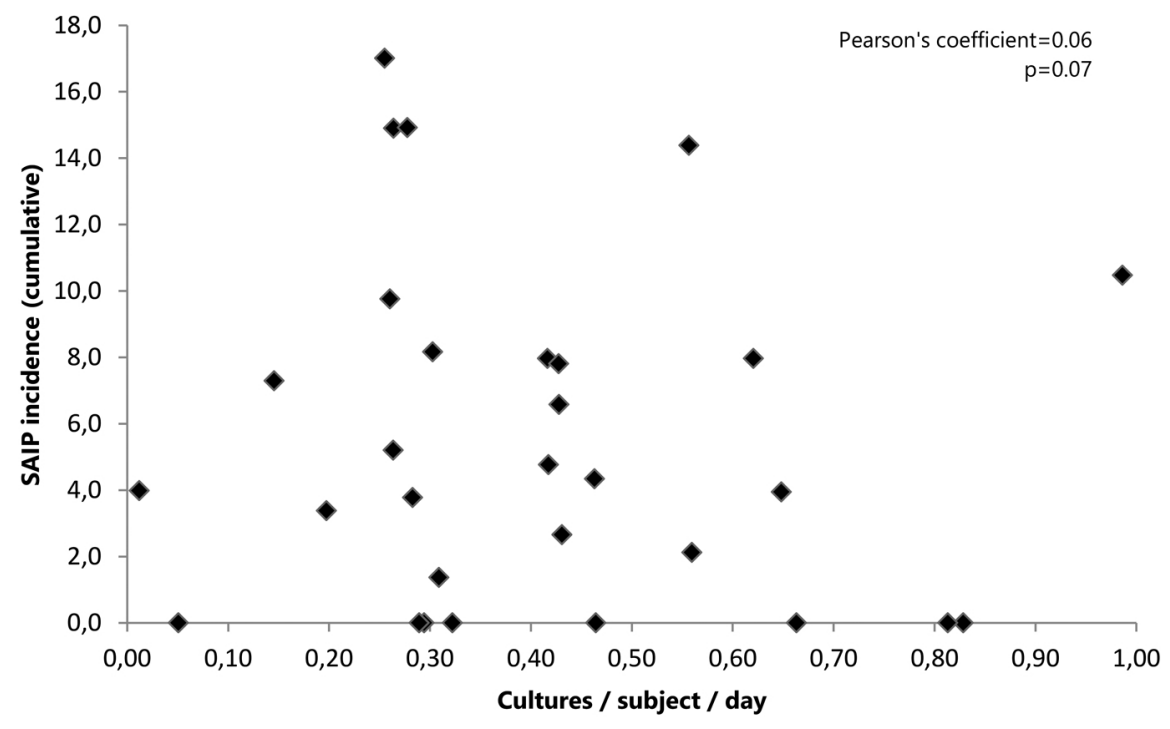

Supplementary Figure SF7: SAIP incidence versus average number of cultures per subject per day over the 30 sites.

Supplementary file S1: Weighting methods

Due to the inclusion criteria of the ASPIRE-ICU study, SA colonized and non-colonized patients were included in the study cohort population at an approximately 1:1 ratio. However, this ratio is approximately 1:3 in the overarching source population. The source population, in being a random sample of the general population, is the population we are interested in making inference on. Therefore, our aim was to recreate the source population by weighting the study cohort subjects with the inverse of the probability of their inclusion in the study cohort[1]. After conducting multiple imputation for missing values in the source population, we fitted a logistic regression model using predictor variables available for the source population, to estimate the inclusion probabilities. The predictor variables are listed in Table 1 below, as well as their characteristics in the source population, study cohort, source minus study cohort, and the weighted study population. There is good agreement between the source and weighted study population; most importantly in the colonization status proportions. The weights were subsequently used in the incidence and risk factor analyses in the accompanying manuscript. 
Table 1.

\begin{tabular}{|c|c|c|c|c|c|}
\hline & level & Source & Study & Source-Study & Weighted \\
\hline$n$ & & 9841 & 1997 & 7844 & 9627 \\
\hline \multirow[t]{3}{*}{ SA Colonization (\%) } & Yes & $2440(24.8)$ & $1007(50.4)$ & $1433(18.3)$ & $2335(24.3)$ \\
\hline & No & $6838(69.5)$ & $990(49.6)$ & $5848(74.6)$ & $7292(75.7)$ \\
\hline & Unknown & $563(5.7)$ & $0(0.0)$ & $563(7.2)$ & $0(0.0)$ \\
\hline \multirow[t]{11}{*}{ Country (\%) } & 1 & $3153(32.0)$ & $344(17.2)$ & $2809(35.8)$ & $3026(31.4)$ \\
\hline & 2 & $550(5.6)$ & $158(7.9)$ & $392(5.0)$ & $510(5.3)$ \\
\hline & 3 & $443(4.5)$ & $127(6.4)$ & $316(4.0)$ & $420(4.4)$ \\
\hline & 4 & $469(4.8)$ & $95(4.8)$ & $374(4.8)$ & $537(5.6)$ \\
\hline & 5 & $721(7.3)$ & $340(17.0)$ & $381(4.9)$ & $710(7.4)$ \\
\hline & 6 & $397(4.0)$ & $205(10.3)$ & $192(2.4)$ & $331(3.4)$ \\
\hline & 7 & $1482(15.1)$ & $338(16.9)$ & $1144(14.6)$ & $1396(14.5)$ \\
\hline & 8 & $352(3.6)$ & $84(4.2)$ & $268(3.4)$ & $339(3.5)$ \\
\hline & 9 & $1302(13.2)$ & $106(5.3)$ & $1196(15.2)$ & $1397(14.5)$ \\
\hline & 10 & $488(5.0)$ & $151(7.6)$ & $337(4.3)$ & $509(5.3)$ \\
\hline & 11 & $484(4.9)$ & $49(2.5)$ & $435(5.5)$ & $452(4.7)$ \\
\hline $\mathrm{AGE}(\operatorname{mean}(\mathrm{SD}))$ & & $62.02(15.69)$ & $62.60(15.92)$ & $61.88(15.64)$ & $62.15(15.12)$ \\
\hline \multirow[t]{2}{*}{ GENDER (\%) } & $\mathrm{F}$ & $3587(36.5)$ & $702(35.2)$ & $2885(36.8)$ & $3507(36.4)$ \\
\hline & M & $6253(63.5)$ & $1295(64.8)$ & $4958(63.2)$ & $6120(63.6)$ \\
\hline \multirow[t]{4}{*}{ REASON (\%) } & MED & $5420(55.1)$ & $1000(50.1)$ & $4420(56.4)$ & $5284(54.9)$ \\
\hline & SURG_CARD & $886(9.0)$ & $124(6.2)$ & $762(9.7)$ & $896(9.3)$ \\
\hline & SURG_OTH & $2306(23.4)$ & $496(24.8)$ & $1810(23.1)$ & $2289(23.8)$ \\
\hline & TRAUMA & $1226(12.5)$ & $377(18.9)$ & $849(10.8)$ & $1158(12.0)$ \\
\hline \multirow[t]{2}{*}{ TRAUMA NEURO (\%) } & NO & $9142(92.9)$ & $1789(89.6)$ & $7353(93.7)$ & $8946(92.9)$ \\
\hline & YES & $699(7.1)$ & $208(10.4)$ & $491(6.3)$ & $681(7.1)$ \\
\hline \multirow[t]{4}{*}{ ADM_YEAR (\%) } & 2015 & $366(3.7)$ & $40(2.0)$ & $326(4.2)$ & $300(3.1)$ \\
\hline & 2016 & $1976(20.1)$ & $316(15.8)$ & $1660(21.2)$ & $1947(20.2)$ \\
\hline & 2017 & $4399(44.7)$ & $954(47.8)$ & $3445(43.9)$ & $4214(43.8)$ \\
\hline & 2018 & $3100(31.5)$ & $687(34.4)$ & $2413(30.8)$ & $3166(32.9)$ \\
\hline \multirow[t]{3}{*}{ Surgery (\%) } & ELEC & $1106(11.2)$ & $174(8.7)$ & $932(11.9)$ & $1169(12.1)$ \\
\hline & EMERG & $2651(26.9)$ & $632(31.6)$ & $2019(25.7)$ & $2593(26.9)$ \\
\hline & NO SURG & $6084(61.8)$ & $1191(59.6)$ & $4893(62.4)$ & $5865(60.9)$ \\
\hline APACHE II SCORE (mean (SD)) & & $20.76(8.90)$ & $20.53(8.58)$ & $20.85(9.03)$ & $21.12(8.87)$ \\
\hline
\end{tabular}

[1] Lee, E.S., Forthofer, R.N, (2006) Analyzing Complex Survey Data: Second Edition, Thousand Oaks, California: SAGE Publications 


$$
\pi \pi
$$




\section{Identifying patients at increased risk for S. aureus ICU pneumonia; results from an international prospective cohort study}

\section{CHAPTER}

leur P. Paling ${ }^{1 *}$

Derek Hazard ${ }^{2 *}$

Claudia Recanatini ${ }^{1}$

Martin Wolkewitz ${ }^{2}$

Hasan S. Jafri ${ }^{3}$

Frangiscos Sifakis ${ }^{4}$

Surbhi Malhotra-Kumar ${ }^{5}$

Herman Goossens ${ }^{5}$

Marc J.M. Bonten ${ }^{1,6}$

Jan A.J.W. Kluytmans ${ }^{1,7}$

and the ASPIRE-ICU Study Team

* both authors contributed equally

Manuscript in preparation

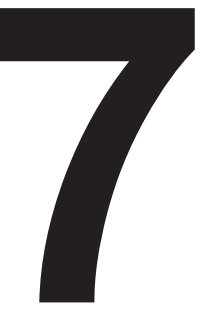

\footnotetext{
1 Julius Center for Health Sciences and Primary Care, University Medical Center Utrecht, Utrecht, Netherlands

${ }^{2}$ Institute of Medical Biometry and Statistics, University Medical Center Freiburg, Freiburg, Germany

${ }^{3}$ Microbial Sciences, R\&D BioPharmaceuticals, AstraZeneca, Gaithersburg, Maryland, United States of America

${ }^{4}$ Boehringer Ingelheim Pharmaceuticals, Inc, Ridgefield, CT, United States of America

${ }^{5}$ University of Antwerp, Antwerp, Belgium

${ }^{6}$ Department of Medical Microbiology, University Medical Center Utrecht, Utrecht, Netherlands

7 Department of Infection Control, Amphia Hospital, Breda, The Netherlands
} 


\section{ABSTRACT}

Background: Identifying patients at risk for Staphylococcus aureus (SA) pneumonia acquired on the intensive care unit (SAIP) is essential for evaluating preventive measures for this infection.

Materials / methods: Data from a prospective observational cohort study in European ICUs were used to build a risk prediction model for SAIP, using information available at ICU admission. SA carriage status of the nose and lower respiratory tract was determined in all patients, and SA carriers were enrolled in a 1:1 ratio to non-carriers. SAIP was defined as pneumonia occurring during ICU stay where SA was isolated from lower respiratory tract or blood. Predefined predictors were selected based on the Akaike's Information Criterion. Performance, calibration, and discrimination of the final model were assessed. Internal validation was performed using bootstrapping.

Results: 131 (6.8\%) out of 1,933 evaluable patients developed SAIP. The final risk prediction model contained the predictors SA colonization status (OR $3.20,95 \% \mathrm{Cl}$ 2.15-4.91, $\mathrm{p}<0.01$ ), neurotrauma (OR 1.67, 95\% Cl 1.01-2.66, $\mathrm{p}=0.04$ ) and prior antibiotic use within the last 2 weeks before ICU admission (OR 0.55, 95\% Cl 0.31-0.90, p=0.03), and performed moderate (area under curve of $0.71, \mathrm{R}^{2}=0.044$ ).

Conclusion: SA colonization status at ICU admission, neurotrauma and prior antibiotic use performed moderately in predicting SAIP. Colonization status was by far the most important determinant and there was practically no value of adding other variables. 


\section{INTRODUCTION}

Intensive care unit (ICU) patients are at high risk for the development of nosocomial complications $^{1-4}$. As most patients are on mechanical ventilation (MV), nosocomial pneumonia diagnosed during ICU stay is a frequently occurring complication ${ }^{5}$. Many preventive measures have been proposed for this infection, such as bed head elevation, subglottic aspiration and oropharyngeal decontamination, which lower the incidence rates in observational studies, but their effectiveness has not been unequivocally demonstrated $^{6}$. For these reasons, new interventions that decrease the occurrence and impact remain warranted. The monoclonal antibody suvratoxumab, developed by MedImmune (now AstraZeneca), is a candidate for prevention of ICU pneumonia caused by Staphylococcus aureus (SA) 7,8. Suvratoxumab neutralizes alpha-toxin (AT), one of SA's major virulence factors. In mice, SA strains lacking the possibility to produce AT do not cause lung infections ${ }^{9}$. In humans, preemptive treatment of SA carriers with suvratoxumab reduced the incidence of SAIP in mechanically ventilated patients in a Phase II randomized controlled trial $(\mathrm{RCT})^{10}$. The current observational study aimed to improve identification (at time of ICU admission) of patients at highest risk for SAIP, by building a prediction model. Such a model might increase trial efficiency of upcoming phase III trial(s) investigating suvratoxumab, or other preventive interventions for SAIP. 


\section{MATERIALS AND METHODS}

\section{Study design and patient population}

ASPIRE-ICU (Advanced understanding of Staphylococcus aureus and Pseudomonas aeruginosa Infections in EuRopE - Intensive Care Units) was a prospective study in 30 hospitals in 11 European countries, between June 2015 and October 2018. A detailed description of the rationale and methods of this study can be found elsewhere ${ }^{11}$. In short, we aimed to include 2,000 adult ICU patients with known SA colonization status at the time of ICU admission in a study cohort, from an overarching source population with an anticipated length of stay (LOS) of $\geq 48$ hours and who were (expected to be) mechanically ventilated within 24 hours after ICU admission. Primary outcomes were derived from the study cohort. In addition, basic surveillance data was used to obtain weighted estimates of predictor prevalence and outcome occurrence in the source population $^{12}$.

Subjects were enrolled in the study cohort in a 1:1 ratio of SA colonized and noncolonized patients, enrolling every SA colonized and the first SA negative patient after every successful inclusion. Colonization status was determined in both nose and LRT upon ICU admission; patients where both could be obtained were eligible for participation in the study cohort. For LRT samples we considered endotracheal aspirate and spontaneously produced sputum, or throat swabs if aspirates and sputum were not available. Enrolled subjects with a diagnosis of SA pneumonia at ICU admission were excluded from this analysis. Other in- and exclusion criteria can be found elsewhere ${ }^{11}$. Study samples were obtained three times weekly in the first week, twice weekly in the three weeks thereafter, and at day of protocol pneumonia diagnosis(e.g. endotracheal aspirates) ${ }^{11}$. Clinical criteria for establishing SAIP diagnosis were collected daily.

All participating ICUs performed routine admission screening for SA colonization in nose and lower respiratory tract at ICU admission. Of each region in Europe, as described by the United Nations, at least one country was included ${ }^{13}$. The study was approved by the institutional review boards or ethical review committees in each country and/or site and registered as ClinicalTrials.gov: NCT02413242.

\section{Primary outcome}

The primary outcome was SAIP occurrence through ICU stay. Protocol pneumonia was defined as any pneumonia during ICU stay developing $\geq 48$ hours after ICU admission and was assessed in two steps. Firstly, four clinical criteria were assessed daily (Table 1). In case of at least one positive answer, a combination of major and minor pneumonia 
criteria were assessed. This was considered to be SAIP in case a lower respiratory tract sample or blood culture was positive for SA in the three days surrounding the date of protocol pneumonia diagnosis. For the full definition we refer to the published study protocol $^{11}$.

Table 1. Criteria scored daily for each subject to assess protocol pneumonia diagnosis

\section{Daily pneumonia score}

- Any new antibiotic use in the last $24 \mathrm{~h}$ $\mathrm{Y} / \mathrm{N}$

- Any new blood cultures drawn within the last 24h $\mathrm{Y} / \mathrm{N}$

- Any new chest X-ray (or CT) taken within the last $24 \mathrm{~h}$ that show a new or $\mathrm{Y} / \mathrm{N}$ worsening infiltrate

- Any other (new) reason occurred in the last $24 \mathrm{~h}$ to suspect a pneumonia $\mathrm{Y} / \mathrm{N}$

If any of the items is scored with 'Yes' ( $\mathrm{Y})$, assessment of all criteria is requested

\section{Laboratory methods}

SA screening samples were processed locally on chromagar plates (Colorex ${ }^{\mathrm{TM}}$ staph aureus, Biotrading) using standardized methods. SA strains were selected on phenotypic criteria (pink or mauve color) and shipped to the central study laboratory. Study samples were frozen locally (at -80 degrees Celsius) and dispatched to the central lab to be analyzed there.

\section{Statistical analysis}

Potential predictors and their management

A list of candidate predictors was defined prior to initiating the study, based on literature and clinical judgment, using variables available at ICU admission, which included; SA colonization status, gender, origin prior to ICU admission (community vs. health care facility), neurotrauma, Acute Physiology Chronic Health Evaluation (APACHE) IV score, prior antibiotic use, diabetes mellitus and body mass index (BMI). Age was excluded, considering this is already included in the APACHE score.

Trauma patients with a Glasgow Coma Scale $<8$ at ICU admission were defined as having neurotrauma. Prior antibiotic use was defined as any systemic antibiotic use during the last two weeks for $\geq 1$ day. BMI and APACHE score were used as continuous variables and measured at ICU admission; it was checked whether fractional polynomials improved model performance ${ }^{14}$. Missing values of any predictors were imputed using imputation techniques ${ }^{15}$. Tests of relevant interactions between SA colonization status and gender, prior antibiotic use, BMI, APACHE IV score and diabetes mellitus were 
performed $(p \text {-value }<0.05)^{16}$. Collinearity between predictors was checked using the variance inflation factor ${ }^{17}$.

\section{Regression model and model performance}

Predictors were selected for logistic regression analysis, using the AIC (Akaike's Information Criterion) and a combination of forward and backward selection ${ }^{18,19}$. Overall model performance was assessed by measuring the Nagelkerke $\mathrm{R}^{2}$, which can be interpreted as the improvement of the final model compared to the null model; and the Brier score, which is the mean squared error of prediction ${ }^{20}$. Calibration of the model was assessed by plotting the observed proportion of events against the predicted risks for groups, defined by ranges of individual predicted risks. Discrimination of the model was evaluated by plotting a receiver operating characteristic (ROC) curve was and calculating the area under the curve (AUC or c-statistic). Internal validation was assessed by performing the identical process on 200 bootstrap samples, then calculating the mean difference in performance ('optimism'), in order to arrive at the optimism corrected performance ${ }^{17}$.

\section{Sensitivity analyses - Competing events}

ICU discharge or death without SAIP are competing events for SAIP. For this reason, as a sensitivity analysis, a Fine and Gray model accounting for competing events was performed, using variable selection methods analogous to that described earlier. By this we calculated subdistribution hazard ratios (SHRs) for SAIP as an alternative measure (by acknowledging the time-dependency) for the odds ratios.

Although region was not considered a predictor, we determined whether inclusion of region during variable selection changed the contents of the final model, because of possible regional differences in infection risks.

All statistical analyses were performed using R version $1.1 .463^{21}$.

\section{RESULTS}

The ASPIRE-ICU database contained 1,997 evaluable study cohort subjects (Figure 1), of which 64 (3.4\%) had SA pneumonia at ICU admission and four had incomplete outcome status, leaving 1,929 patients for this analysis. Baseline characteristics can be found in Table 2. In all, 304 (15.7\%) patients developed protocol pneumonia, of which 131 (6.8\%) were classified as SAIP. 


\section{Predictors of SAIP}

No relevant interactions or multicollinearity between potential predictors were observed. Out of 8 variables, the following predictors were selected for the final multivariate risk prediction model: SA colonization status (OR 3.20,95\% Cl 2.15-4.91, $\mathrm{p}<0.01$ ), neurotrauma (OR 1.67, 95\% $\mathrm{Cl} 1.01-2.66, \mathrm{p}=0.04$ ) and antibiotic use in the last two weeks prior to ICU stay (OR 0.55, 95\% CI 0.31-0.90, p=0.03, Table 3). The use of fractional polynomials for BMI or APACHE IV did not improve the model.

\section{Model performance}

The improvement seen in the final model when compared to a model without any variables (or the explained variation), as indicated by the Nagelkerke $\mathrm{R}^{2}$ was 0.068 . The Brier score was 0.062 (scaled Brier 14\%). The risk to develop SAIP among those with all three risk factors ( $n=113$ ) was $15 \%$, which is almost five times higher than the risk of $3.2 \%$ for those ( $n=282$ ) without any risk factor. However, the majority of patients in our study cohort ( $n=1,534,79.5 \%$ ) had 1 or 2 risk factors, associated with a risk of $7.4-11.5 \%$.

The calibration plot (Figure 2) demonstrates moderate agreement between the observed events and the predicted risks by ranges of individual predicted risks. Discrimination of the model was moderate, with an area under the ROC curve of 0.74 (Figure 3 and 4). 


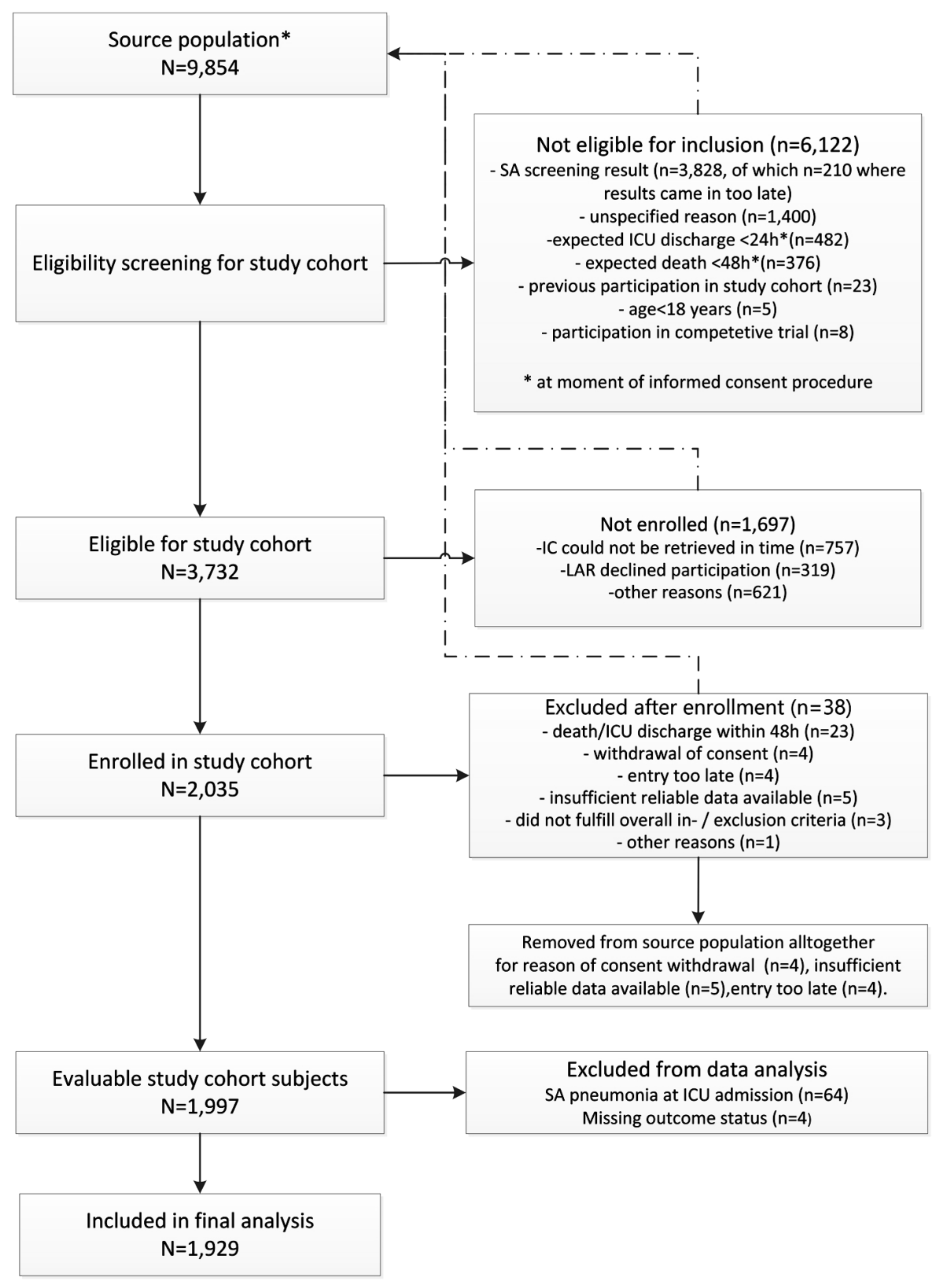

Figure 1. Flowchart of patients / subjects within ASPIRE-ICU

$S A=S$. aureus, $I C U=$ intensive care unit, $I C=$ informed consent, $L A R=$ legally accepted representative

* Final evaluable number of patients in source population is 9,841 , because of the 13 subjects that were removed afterenrollment in study cohort. 
Table 2. Baseline characteristics.

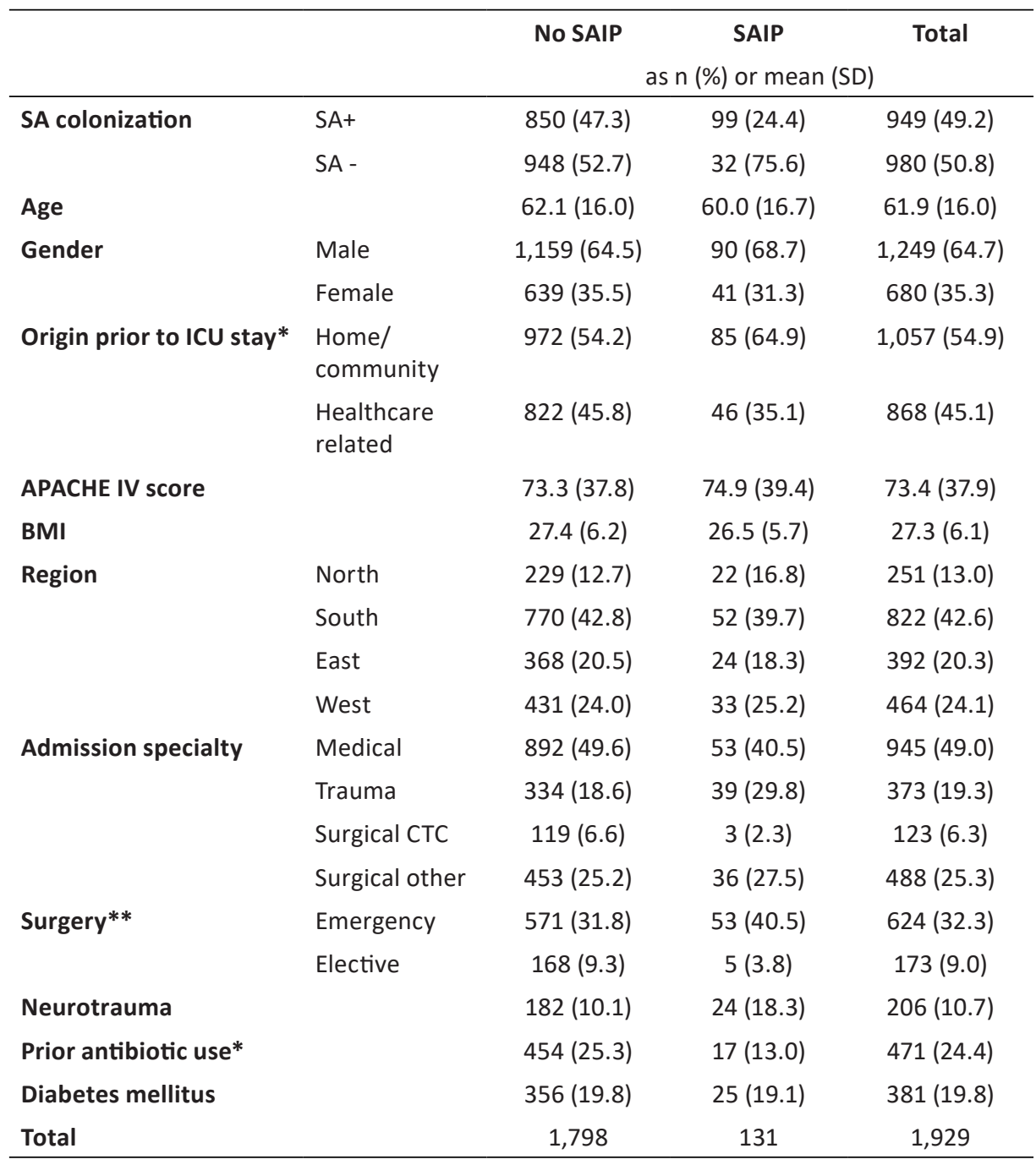

$S A I P=S A I C U$ pneumonia, $S A=S$. aureus,$S D=$ standard deviation, $I C U=$ intensive care unit, $A P A C H E=$ Acute Physiology, Age, Chronic Health Evaluation, BMI= body mass index, CTC= cardiothoracic.

*After imputation of unknown values. ${ }^{* *}$ In case a trauma subject needed surgery related to this trauma, this was assumed to be emergency surgery. 
Table 3. Multivariate logistic regression analysis

\begin{tabular}{lll}
\hline & Adjusted OR $(95 \% \mathrm{Cl})$ & p-value \\
\hline Gender* & Not included & \\
APACHE IV score† & Not included & \\
SA colonization & $3.20(2.15-4.91)$ & $<0.001$ \\
BMI† & Not included & \\
Origin prior to ICU stay & Not included & \\
Neurotrauma & $1.67(1.02-2.66)$ & 0.04 \\
Prior antibiotic use & $0.55(0.31-0.90)$ & $\mathbf{0 . 0 3}$ \\
Diabetes mellitus & Not included & \\
\hline
\end{tabular}

$\mathrm{OR}=$ odds ratio, $\mathrm{Cl}=$ confidence interval, $\mathrm{APACHE}=$ Acute Physiology, Age, Chronic Health Evaluation, $\mathrm{SA}=S$. aureus, $\mathrm{BMI}=$ body mass index, $\mathrm{ICU}=$ intensive care unit. Bold= significant at 0.05 level.

*Female is reference category, tOR per point / year of $\mathrm{kg} / \mathrm{M}^{2}$ increase, "home is reference category.

\section{Internal validation}

Internal validation using 200 bootstrap samples, yielded an optimism corrected $\mathrm{R}^{2}$ of 0.044 , Brier Score of 0.063 and AUC of 0.71 .

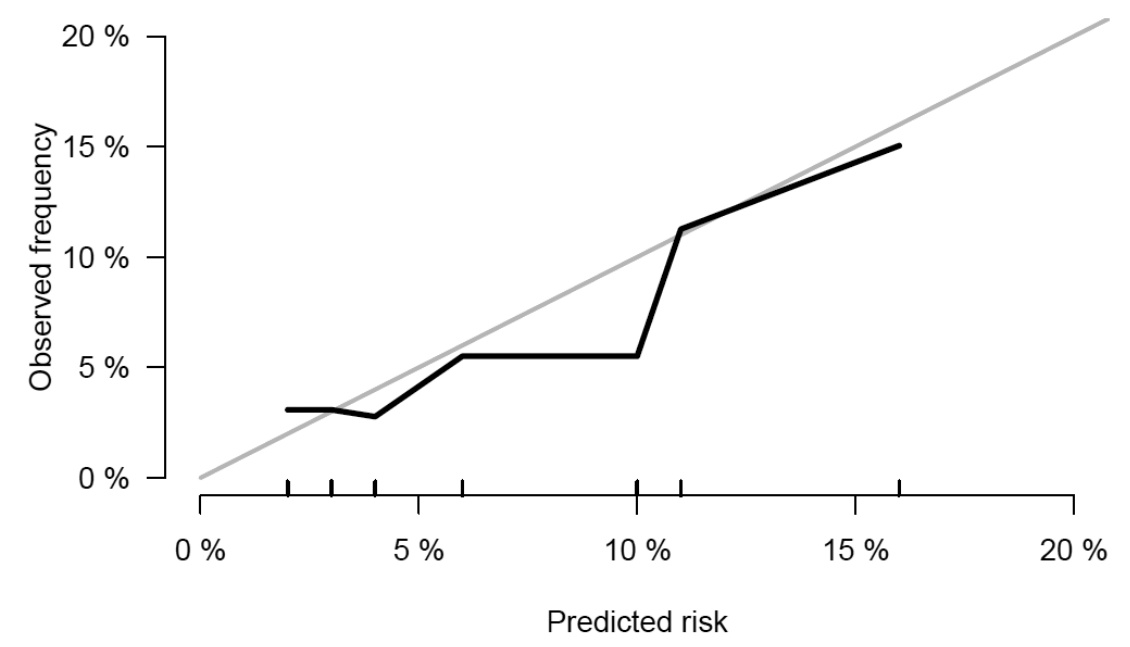

Figure 2. Calibration plot of final model, showing observed proportion of events vs. predicted risks 


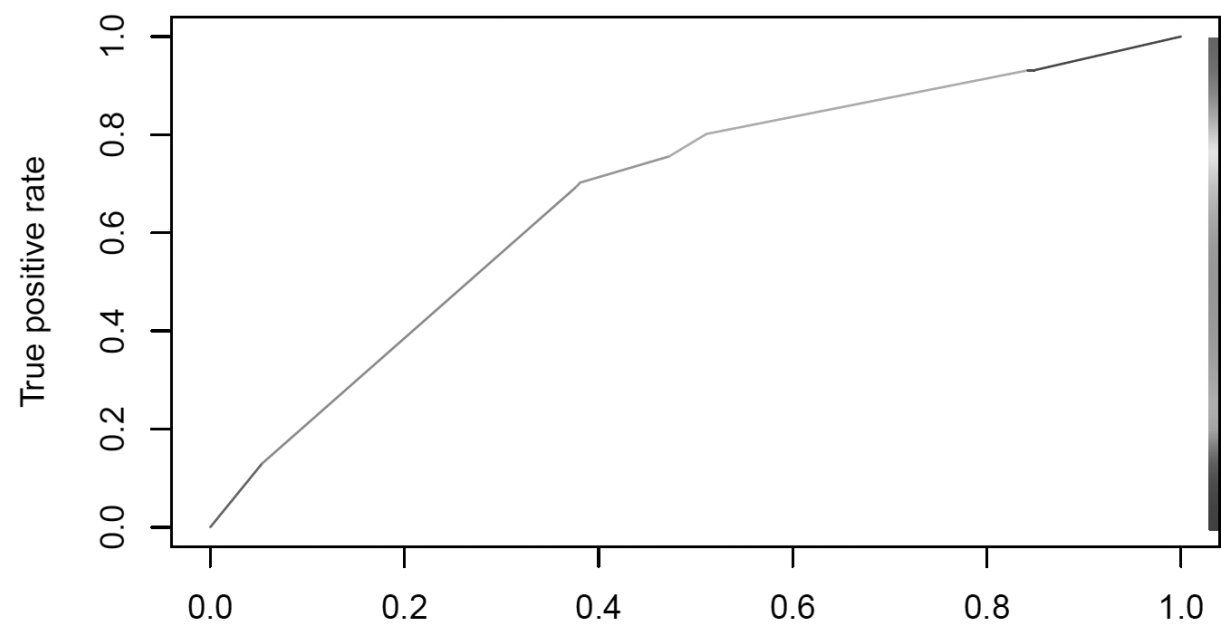

False positive rate

Figure 3. ROC curve of final model, with an AUC of 0.74 .

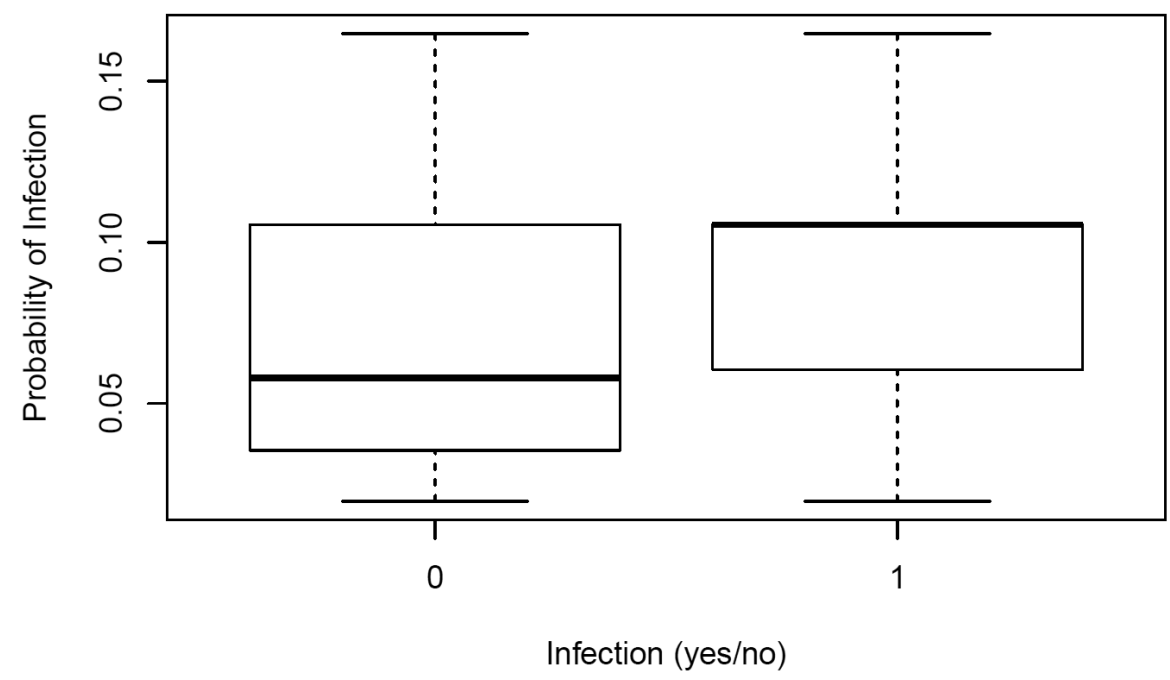

Figure 4. Boxplot showing distribution of prediction risks stratified for groups with/without primary outcome.

Bold lines indicate median probability. 


\section{Sensitivity analysis}

\section{Competing risks}

A Fine and Gray model accounting for competing events, using analogous methods for variable selection, selected the same predictors in the final model and did not show relevant changes of coefficients (maximum observed change was 3.6\%) when compared to the logistic regression model used.

\section{Predictive value of region}

Including region as a predictor in the variable selection did not change the final model; in fact, region was the first variable to be dropped.

\section{DISCUSSION}

A risk prediction model containing the variables SA colonization status at ICU admission, neurotrauma and antibiotic use in the last two weeks before ICU admission performed moderately when predicting SAIP during ICU stay. SA colonization status is the most important predictor of all tested variables; in terms of effect size as well as prevalence.

Table 4. Enrichment scenarios for SAIP intervention trials

\begin{tabular}{lllllll}
\hline Scenario & Prevalence & Crude RR & SAIP \% & Size TP & Size SP & NNE 1 SAIP \\
\hline Total population & 1 & ref & $5.3 \%$ & 1 & 1 & 18.9 \\
SA colonized (1) & 0.23 & 3.7 & $11.8 \%$ & 0.45 & 2.0 & 8.5 \\
Neurotrauma (2) & 0.07 & 2.3 & $10.9 \%$ & 0.49 & 6.9 & 9.2 \\
No prior AB (3) & 0.76 & 1.6 & $5.8 \%$ & 0.91 & 1.2 & 17.2 \\
1 or 2 & 0.28 & 3.6 & $10.9 \%$ & 0.49 & 1.7 & 9.2 \\
\hline
\end{tabular}

Prevalence: prevalence of the predictor in the population. RR: Relative risk of the predictor for occurrence of SAIP. SAIP \%: event risk of the included population. Size TP (trial population): number of randomised subjects needed relative to running an RCT in the total population. Size SP (screening population): number of patients to be screened for inclusion relative to running an RCT in the total population. NNE 1 SAIP: number of subjects needed to enrol, to find 1 case of SAIP.

We extrapolated these results to the overarching source population, where roughly $25 \%$ of the patients are colonized with SA upon ICU admission, and in which outcome incidence was estimated using weighting procedures described elsewhere ${ }^{12}$. Here, around $20 \%$ of the patients had 0 risk factors (risk of SAIP $~ 3.5 \%$ ), $80 \%$ had $\geq 1$ (SAIP risk 5.7\%), and $24 \%$ had $\geq 2$ (SAIP risk 12.1\%). From the perspective of trial efficiency, we were interested to find the optimal population for a future preventive intervention 
study. Using the predictors identified here, one can think of several enrichment strategies for upcoming intervention studies (Table 4). This shows for example that even though neurotrauma is associated with a risk close that for SA colonized, it is a less attractive selection method for trial inclusion, as it involves a relatively small ICU population. On the other hand, colonization requires screening and neurotrauma is identified without additional diagnostic procedures. Nevertheless, screening for SA is in our opinion more attractive, considering carriage occurs in $23 \%$ of all ICU patients and is associated with a substantially higher risk. Screening costs are relatively low, whereas the costs per enrolled subject in studies can be as high as $\$ 6,000$. Based on the methods used in our study, with screening costs of around $\$ 4$ per patient, inclusion of SA colonized is an easy way to save money at acceptable additional time investment. To further substantiate this, we calculated model performance statistics for a model with only carriage; Nagelkerke $\mathrm{R}^{2}$ was 0.053 and AUC was 0.78 ; in other words, explained variation was slightly lower, but discrimination better.

Risk prediction models targeting SAIP have not yet been developed. A model trying to predict methicillin resistant SA (MRSA) colonization and/or infection, performed comparably to our model ${ }^{22}$. One study assessing risk factors for SA nosocomial pneumonia on the ICU demonstrated coma to be an important predictor, which is in line with our finding ${ }^{23}$. Lastly, a previous study performed within this consortium also revealed colonization to be the most important risk factor ${ }^{24}$.

As any analysis, this one also has several limitations. Firstly, no regional effect could be taken into account, considering the practical obstacle that the European regions considered in this study are not deemed homogenous enough to warrant extrapolation to a complete region. Even though we observed no improvement of the model when adding region, external validation in any country of interest is advised before local performance can be assumed. Furthermore, our screening cultures did not include any quantification of bacterial load, while this was found to be of added value in the prediction of SA surgical site infection and ventilator-associated pneumonia ${ }^{25,26}$. Last but not least, the definition of SAIP used in this study is debatable, as it appears to be influenced by local culture practices, which differed between participating countries (data shown elsewhere) ${ }^{12}$. In short, SAIP occurrence in this study is likely to be overestimated, which means that the clinical relevance of preventing this outcome, which may also include non-SAIP cases has not yet been established, but remains to be proven by upcoming clinical trials. 
To improve the prediction of SAIP there is a potential added value of other biomarkers, besides SA colonization. For example the antibody titers against virulence factors, e.g. alphatoxin. Results of the earlier mentioned RCT investigating suvratoxumab are in line with this. It is unlikely however, for an assay like anti-AT to become available throughout Europe anytime soon, in order to add anything to the identification of patients at risk. For this reason we feel that future studies evaluating preventive measures for SAIP would be clever to stick to the 'good old' SA colonization when selecting trial populations.

\section{CONCLUSION}

SA colonization status at ICU admission is the most important predictor for SAIP. Neurotrauma and prior antibiotic use are also independent predictors, but they do not contribute much to identify patients at risk and are less useful to increase the efficiency of trials or preventive treatment strategies.

ASPIRE-ICU study team: Omar Ali, Martin Wolkewitz, Alexey Ruzin, Leen Timbermong, Christine Lammens, Sebastiaan Hullegie, Darren Troeman, Denise van Hout, Daniël Prins, Rubana Kalyani, Mark Eickhoff, Kathryn Shoemaker, Tuba Vilken, Jelle Vlaeminck, Jasmine Coppens, Thomas van der Schalk, Basil Britto Xavier, Atanas Temelkov, Nikolay Petrov, Rossitza Vatcheva, Michal Drab, Jaromir Vajter, Kadri Tamme, Muriel Fartoukh, Alain LePape, Mickael Landais, Gaetan Plantefève, Evelina Tacconelli, Achim Kaasch, Róbert Jurkinya, Dora Iványi, Miranda van Rijen, Olaf Cremer, Biljana Carevic, Jasna Jevdjić, Dolores Escudero, Miguel Sanchez Garcia, Cristina Prat-Aymerich, Borja Suberviola-Cañas, Angel Arenzana-Seisdedos Hürrem Bodur, Cenk Kirakli, Ilkay Bozkurt, David Pogson, Sandra Long, Matt Wise.

Acknowledgements: We would like to thank Rianne Jahja, Jelle Lyskawa, Nicolette van der Sluis, Janet Couperus, Nienke Cuperus, Elien Burgers of the operational team of the University Medical Center of Utrecht; Liesbeth Bryssinck, Gert Leten, Anouk Vanderstraeten, Sofie Van Mieghem, Sabine Chapelle and Kitty Gys of the operational team of University of Antwerp, everybody of the operational teams of Seville and Limoges, all local investigators, monitors, other staff and participating patients.

Funding: This research project receives support from the Innovative Medicines Initiative Joint Undertaking under grant agreement no. 115523 and 115737 resources which are composed of financial contribution from the European Union Seventh Framework Programme (FP7/2007-2013) and EFPIA companies in kind contribution. 


\section{REFERENCES}

1. Koulenti D, Tsigou E, Rello J. Nosocomial pneumonia in 27 ICUs in Europe: perspectives from the EU-VAP/CAP study. Eur J Clin Microbiol Infect Dis. 2017;36(11):1999-2006. doi:10.1007/ s10096-016-2703-z

2. Mnatzaganian $\mathrm{G}$, Galai N, Sprung $\mathrm{CL}$, et al. Increased risk of bloodstream and urinary infections in intensive care unit (ICU) patients compared with patients fitting ICU admission criteria treated in regular wards. J Hosp Infect. 2005;59(4):331-342. doi:10.1016/j.jhin.2004.07.028

3. Laupland KB, Zygun DA, Davies HD, Church DL, Louie TJ, Doig CJ. Incidence and risk factors for acquiring nosocomial urinary tract infection in the critically ill. J Crit Care. 2002;17(1):50-57. http://www.ncbi.nlm.nih.gov/pubmed/12040549. Accessed July 24, 2019.

4. Dereli N, Ozayar E, Degerli S, Sahin S, Koç F. Three-Year Evaluation of Nosocomial Infection Rates of the ICU. Brazilian J Anesthesiol (English Ed. 2013;63(1):73-78. doi:10.1016/j. bjane.2012.03.004

5. Guzmán-Herrador B, Díaz Molina C, Allam MF, Fernández-Crehuet Navajas R. Underlying illness severity and outcome of nosocomial pneumonia: prospective cohort study in intensive care unit. J Hosp Infect. 2014;86(1):53-56. doi:10.1016/j.jhin.2013.09.014

6. Bouadma L, Wolff M, Lucet J-C. Ventilator-associated pneumonia and its prevention. Curr Opin Infect Dis. 2012;25(4):395-404. doi:10.1097/QCO.0b013e328355a835

7. Sause WE, Buckley PT, StrohI WR, Lynch AS, Torres VJ. Antibody-Based Biologics and Their Promise to Combat Staphylococcus aureus Infections. Trends Pharmacol Sci. 2016;37(3):231241. doi:10.1016/j.tips.2015.11.008

8. François B, Chastre J, Eggiman P, et al. The SAATELLITE and EVADE Clinical Studies Within the COMBACTE Consortium: A Public-Private Collaborative Effort in Designing and Performing Clinical Trials for Novel Antibacterial Drugs to Prevent Nosocomial Pneumonia: Table 1. Clin Infect Dis. 2016;63(suppl 2):S46-S51. doi:10.1093/cid/ciw245

9. Bubeck Wardenburg J, Bae T, Otto M, DeLeo FR, Schneewind O. Poring over pores: $\alpha$-hemolysin and Panton-Valentine leukocidin in Staphylococcus aureus pneumonia. Nat Med. 2007;13(12):1405-1406. doi:10.1038/nm1207-1405

10. François B, Garcia Sanchez M, Eggimann P, et al. Efficacy and Safety Profile of Suvratoxumab, a Novel Anti-Staphylococcus aureus Monoclonal Antibody: Results of the SAATELLITE Study in Mechanically Ventilated Intensive Care Unit Patients. In: 29th European Congress of Clinical Microbiology \& Infectious Diseases, Amsterdam. ; 2019. 
11. Paling FP, Troeman DPR, Wolkewitz M, et al. Rationale and design of ASPIRE-ICU: A prospective cohort study on the incidence and predictors of Staphylococcus aureus and Pseudomonas aeruginosa pneumonia in the ICU. BMC Infect Dis. 2017;17(1). doi:10.1186/s12879-017-2739-4

12. Paling FP, Hazard D, Bonten MJM, et al. Staphylococcus Aureus Colonization and the Occurrence of ICU Pneumonia; ASPIRE-ICU, a Prospective International Cohort Study; 2019.

13. United Nations Statistics Division. Standard Country or Area Codes for Statistical Use.; 1999:54. https://unstats.un.org/unsd/methodology/m49/. Accessed October 29, 2019.

14. Royston P, Ambler G, Sauerbrei W. The use of fractional polynomials to model continuous risk variables in epidemiology. Int J Epidemiol. 1999;28(5):964-974. doi:10.1093/ije/28.5.964

15. Sterne JAC, White IR, Carlin JB, et al. Multiple imputation for missing data in epidemiological and clinical research: potential and pitfalls. BMJ. 2009;338(jun29_1):b2393. doi:10.1136/bmj. b2393

16. Kleinbaum DG, Klein M. Logistic Regression: A Self-Learning Test. New York: Springer; 2010.

17. Sheather SJ. A Modern Approach to Regression with R. New York: Springer; 2009.

18. Venables VN, Ripley BD. Modern Applied Statistics with S. 4th ed. Springer; 2002.

19. Steyerberg EW. Clinical Prediction Models. Springer; 2009.

20. Harrel F. Regression Modeling Strategies; With Applications to Linear Models, Logistic Regression, and Survival Analysis. New York: Springer; 2001.

21. R Core Team. R: A Language and Environment for Statistical Computing. R Foundation for Statistical Computing. 2017. https://www.r-project.org.

22. Callejo-Torre F, Eiros Bouza JM, Olaechea Astigarraga P, et al. Risk factors for methicillinresistant Staphylococcus aureus colonisation or infection in intensive care units and their reliability for predicting MRSA on ICU admission. Le Infez Med. 2016;24(3):201-209. http:// www.ncbi.nlm.nih.gov/pubmed/27668900. Accessed November 3, 2019.

23. Rello J, Quintana E, Ausina V, Puzo C, Net A, Prats G. Risk Factors for Staphylococcus aureus Nosocomial Pneumonia in Critically III Patients. Am Rev Respir Dis. 1990;142(6_pt_1):13201324. doi:10.1164/ajrccm/142.6_Pt_1.1320

24. Paling FP, Wolkewitz M, Bode LGM, et al. Staphylococcus aureus colonization at ICU admission as a risk factor for developing S. aureus ICU pneumonia. Clin Microbiol Infect. 2017;23(1). doi:10.1016/j.cmi.2016.09.022 
25. Kluytmans J, van Belkum A, Verbrugh H. Nasal carriage of Staphylococcus aureus: epidemiology, underlying mechanisms, and associated risks. Clin Microbiol Rev. 1997;10(3):505-520. http://www.pubmedcentral.nih.gov/articlerender. fcgi ?artid=172932\&tool=pmcentrez\&rendertype=abstract. Accessed September 8, 2014.

26. Kabak E, Hudcova J, Magyarics Z, et al. The utility of endotracheal aspirate bacteriology in identifying mechanically ventilated patients at risk for ventilator associated pneumonia: a single-center prospective observational study. BMC Infect Dis. 2019;19(1):756. doi:10.1186/ s12879-019-4367-7 



\section{PART THREE}

CONCLUSIONS 


$$
\pi \pi
$$


General discussion

CHAPTER

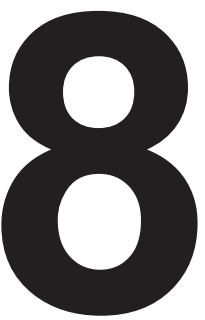


This thesis aimed to assess the occurrence of pneumonia acquired in the intensive care unit (ICU) caused by Staphylococcus aureus and Pseudomonas aeruginosa in Europe. Furthermore, it determined risk factors that will help identifying patients at higher risk for S. aureus ICU pneumonia or surgical site infection (SSI). To a lesser extent, this was also done for $P$. aeruginosa ICU pneumonia. The role of prior colonization in the development of these healthcare-associated infections (HAIs) received particular attention. In this chapter, the findings are presented in a broader context and implications for future research are discussed.

\section{What was done by others - Carriage risks reports throughout history}

The role of carriage in the disease pathogenesis of nosocomial S. aureus (SA) infections was recognized already more than 60 years ago, when observations by Williams demonstrated a three times higher occurrence of staphylococcal sepsis in nasal carriers after surgery ${ }^{1}$. Shortly thereafter, attempts (at that time unsuccessful) were made to decrease infection rates by using decolonizing interventions, like treatment with neomycin nasal cream ${ }^{2}$. Over the years, it became clear that carriage status was not only important for surgical patients, but also for other populations, for example for patients infected with human immunodeficiency virus (HIV), where the odds ratio was are around 4 for nasal carriers to develop SA infection ${ }^{3}$. But also patients undergoing liver transplantation or those receiving renal dialysis were at higher risk for SA infection when carrying SA in the nose $\mathrm{e}^{4-6}$. In the mid-nineties, the first reports were written on the risks of carriage for ICU patients, one of which presented a very high relative risk (RR) of 59.6 (95\% confidence interval [CI] 20.4-184.3) for carriers compared to non-carriers to acquire SA infection during ICU stay. Another study by Pujol et al from the same period, reported a lower RR of 12.4 (95\% Cl 5.3-29.0), also in ICU patients ${ }^{7,8}$. Corbella's discrepant finding, however, is most likely the result of a rather unusual definition of the exposed and unexposed, as they appear to compare patients who were SA positive at ICU admission to patients who stayed SA negative throughout their entire ICU stay (instead of comparing to those SA negative at ICU admission). If one calculates the RR comparing SA carriers and non-carriers at the time of ICU admission, this would yield a crude RR of 10.3 (95\% confidence interval 5.6-18.9), which is comparable to the crude risk in other populations, as well as to that of Pujol et al. In any case, both studies demonstrate an increased risk for SA carriers to acquire SA infection when hospitalized on the ICU.

Up until this moment, no specific analyses were performed to assess the contribution of carriage in relation to contracting SA pneumonia, apart from a few (very) small 
subgroup analyses, some of which in the studies described in the previous paragraph. This changed in 1999 when Campbell described a population of (ICU) trauma patients where the risk for nasal carriers (carriage being assessed $<72 \mathrm{~h}$ after ICU admission) to acquire SA pneumonia was higher than for non-carriers ( $R R$ 4.1, 95\% $\mathrm{Cl} 1.9-8.7, \mathrm{p}<0.001$ ). Additionally, the study also revealed isolated head injury to be a potential risk factor for SA pneumonia within SA carriers (RR 2.6, 95\% Cl 1.2-5.5, $\mathrm{p}=0.01$ ). Unfortunately, this study suffered from a low number of events and did not perform time to event or multivariate analyses ${ }^{9}$. In the years following, there was one more notable study on SA pneumonia in ICU patients, which was the study of Rocha in 2013, performed in Brazil $^{10}$. This study assessed the (univariate) association between SA nasal carriage at ICU admission and SA ventilator-associated pneumonia, demonstrating an OR of 2.7 $(95 \% \mathrm{Cl} 1.0-7.1)$ for carriers.

In summary, several studies have been performed to assess the role of SA carriage in the development of nosocomial SA infections. However, when reviewing the literature, it is striking to see that few used multivariate analysis, thus accounting for over- or underrepresentation of other possible risk factors and / or confounders in (non-) colonized patients. Apart from the obvious reasons to do so if one is interested in causality, this is also relevant for prediction purposes to understand the distribution of risks and to be able to interpret (and potentially extrapolate) the results. Furthermore, over the last decade, consensus was reached that standard time-to-event Kaplan Meier curves are likely to overestimate the cumulative risk of nosocomial infection in the presence of so-called competing events ${ }^{11}$. Competing events are events which prevent the event of interest from happening. Take for example ICU pneumonia. Acknowledged competing events here are ICU discharge alive without ICU pneumonia or death without ICU pneumonia, because once a patient is no longer in the ICU, he/she cannot acquire ICU pneumonia anymore. In classic survival analyses like Kaplan Meier, such patients are censored. One of the major assumptions of censoring, however, is that the instantaneous risk to acquire the event after censoring remains the same. Clearly, in our situation, if a patient dies or is discharged from ICU before ICU pneumonia and the subsequent daily risk to acquire ICU pneumonia drops to 0 , this is not the case. In other words, if the (risk of acquiring a) competing event is not taken into account, the risk estimates are likely to be biased. Unfortunately, none of the analyses described in this paragraph performed competing risks analysis.

When shifting shortly to importance of Pseudomonas aeruginosa (PA) carriage, it should be mentioned that the average carriage rate of this bacterium at ICU admission is lower (below 20\%), and acquisition typically occurs during ICU stay ${ }^{12,13}$. Additionally, while for 
SA the dominant residing niche is the nose, the preferred body site for PA carriage is the intestine ${ }^{12,14}$. For this reason, PA screening cultures are usually taken from rectum, perirectal or perianal areas and sometimes stool. Reports on (intestinal) colonization as a risk factor for subsequent nosocomial PA infections in the ICU are fewer than for SA, but point in the same direction, with incidence rate ratios (or comparable measures) between 6 and $15^{13,15}$. Although here multivariate analyses have been used, the quality and comparability of studies is varying and competing events were not accounted for. Combining this with the quantity of the evidence, the knowledge gap is clear.

\section{What we did (differently) - summary of this thesis' findings}

A major underlying driver of the research described in this thesis, is the clinical evaluation of the monoclonal antibodies suvratoxumab and MEDI3902 as a new preventive therapy for SA and PA infection, respectively. Clinical trials are needed for evaluation of its efficacy, which ideally are performed on a study population with a reasonably high incidence of SA infection, in order to limit the required number of patients to reliably demonstrate efficacy. The research described in this thesis aids towards identification of this ideal study population.

Realizing that the available evidence was not obtained using current state-of-the-art standard statistical methods, the starting exercise of this thesis was to (re-)evaluate already available data of recent age, arbitrarily using a cut-off of 10 years. This was described in chapters 2-4. Additionally, we performed two prospective, international studies (one for SA and PA ICU pneumonia, one for SA SSI), where colonization status would be assessed in a standardized manner with rigorous follow-up for the infectious outcome. These studies were specifically designed to assess the role of colonization status, while at the same time being able to determine many other risk factors (including a large number of biomarkers). One of these studies, including its first results, is described in chapters 5-7.

For the first 'retrospective' part of the thesis, where the analyses were performed on existing databases, we collected databases containing information on colonization status as well as (time to) infection using a systematic approach. These were subsequently analyzed using the competing risks analysis described earlier. By doing this, not only the role of carriage (and other risk factors), but also any impact of competing events on risk estimation of SA infection was taken into account. In chapter $\mathbf{2}$, we analyzed data from ICU patients collected from two prospective cohorts. Diagnoses were made using objective definitions (including at least semi-quantitative cultures) and cross-validated by research physicians of the projects. We assessed the occurrence of ICU acquired 
pneumonia caused by SA while acknowledging the competing events ICU discharge alive and death within ICU without SA ICU pneumonia. As indicated earlier, the most important risk factor we intended to quantify in this analysis was SA colonization status at ICU admission. We found that for the two cohorts colonization status was assessed in $58 \%$ and $80 \%$ of the cases, mainly in lower respiratory tract, and was found positive in $12.7 \%$ and $12.8 \%$ of those tested. The most important reason for non-testing was an expected length of stay $\leq 48$ hours, which was more often the case in one of the cohorts because of case-mix differences (e.g. larger proportion of surgical patients). SA ICU pneumonia occurred in $1.1 \%$ and $1.3 \%$ of the patients, with SA colonization at ICU admission being the most important risk factor for its development, finding a subdistribution hazard ratio (SHR) of $9.6(95 \% \mathrm{Cl} 5.3-17.2, \mathrm{p}<0.001)$ and 14.5 (95\% $\mathrm{Cl} 7.2-29.2, \mathrm{p}<0.001)$ for colonized vs. non-colonized patients, performing a Fine and Gray competing risks analysis taking into account competing events. This result can be interpreted as; patients carrying SA at ICU admission have a daily risk during ICU stay of developing SA ICU pneumonia that is on average 9.6 and 14.5 times higher than patients who are known to be non-colonized at ICU admission. The only other risk factor found was mechanical ventilation at ICU admission, with a SHR of $3.7(95 \% \mathrm{Cl} 1.0-12.1, \mathrm{p}=0.03)$ and 7.0 (95\% Cl 3.0-16.4, p<0.001) in the two cohorts, respectively. Unfortunately, due to the low number of events in these cohorts, we were limited towards the number of risk factors being able to test.

The notably increased SHR for colonized patients was also found for $P$. aeruginosa (colonization being assessed in ETA, oropharynx and/or rectum), being $8.8(95 \% \mathrm{CI}$ $5.0-15.7, p<0.001$ ) instead of 14.5 , as described in chapter 4 . The PA analysis was performed in only one of the cohorts described in chapter 2 , because of the systematic use of selective decontamination of the digestive tract in the other cohort, and its expected impact on the incidence of PA ICU pneumonia. The difference in SHR does not necessarily imply a lower impact of colonization status on disease risk, as PA colonization is more typically acquired during ICU stay, which this was not taken into account in this analysis. The lower PA colonization prevalence at ICU admission of 9.2\% in those tested in combination with the (to SA) comparable cumulative incidence of PA ICU pneumonia of $1.3 \%$ at the end of ICU stay would fit with this reasoning. Furthermore, the median time to PA ICU pneumonia was 7 days which, being one day more than for SA ICU pneumonia (SAIP), may as well suggest a growing prevalence of PA colonization during ICU stay (not measured here), by this leading up to the incidence comparable to SAIP, but taking slightly more time. Mechanical ventilation at ICU admission was similarly associated with a higher occurrence of PA ICU pneumonia (SHR being 5.3, 95\% Cl 2.7- 
10.5, $p<0.001)$. Overall, the results found in the analyses described in the first two chapters yield risks for colonized patients that are comparable to most of the previous reports from literature.

In chapter $\mathbf{3}$ we made a small sidestep by performing a post-hoc analysis into carriage risks for the surgical patient population. We did this by describing the development of a risk prediction model for prediction of SA SSI and/or bacteremia within the first 90 days after cardiothoracic surgery. For the analysis we used data of a previously performed randomized controlled trial, which investigated the effect of a vaccine against SA on the prevention of SA SSI and/or bacteremia ${ }^{16}$. This large, well-organized and very complete database contained many variables, including SA colonization status preceding surgery. Prior to analysis we chose to investigate the following risk factors in the prediction model: SA colonization status prior to surgery, pre-operative antibiotic use, diabetes mellitus, type of cardiothoracic procedure, body mass index (BMI), age, and gender. Knowing that the vaccine had no significant effect on the development of SA SSI we included the complete study population in our analysis. We used a logistic regression model, because of completeness of the dataset and the binary nature of the outcome, collected at a fixed time point after surgery. Acknowledging the importance of competing events, a competing risks analysis accounting for death prior to SA SSI/ bacteremia was performed as a sensitivity analysis, to assess whether competing events influenced the cumulative risk of the event and/or or the estimated odds ratios for SA colonization and/or other risk factors. We found that prior SA colonization was the main independent risk factor for the development of SA SSI/bacteremia within 90 days after surgery, with an odds ratio of $3.1(95 \% \mathrm{Cl} 2.2-4.2, \mathrm{p}<0.001)$. Other independent risk factors (identified via forward selection methods) were BMI, diabetes mellitus and type of procedure (undergoing coronary artery bypass grafting being associated with an increased odds ratio compared to other procedures). The final risk prediction model with these variables performed satisfactorily in its prediction and remained stable after internal validation using bootstrapping. However, we deemed this model not to be suitable for use in clinical practice as less than $3 \%$ of the patients had a risk of more than $10 \%$ to develop the outcome, which is understandable with the overall low occurrence of SA SSI/bacteremia (2.1\%).

Then, from chapter 5 onwards, the thesis discusses ASPIRE-ICU (Advanced understanding of Staphylococcus aureus and Pseudomonas aeruginosa Infections in EuRopE - Intensive Care Units), the previously announced prospective study designed specifically to assess the occurrence and risk factors of SA and PA ICU pneumonia in Europe. The low occurrence of SA ICU pneumonia described in chapter 2, led to enrolling ASPIRE- 
ICU patients based on the two most important risk factors identified; SA colonization status and mechanical ventilation at ICU admission. Consequently, in ASPIRE-ICU we aimed to enroll 2,000 subjects on mechanical ventilation at ICU admission or (expected to be) shortly thereafter of which 50\% SA colonized and 50\% non-colonized. Knowing that mechanical ventilation at ICU admission was also a risk factor for $P$. aeruginosa ICU pneumonia (chapter 4), this enrichment strategy was simultaneously deemed to increase the event rate of $P$. aeruginosa ICU pneumonia. Further enrichment based on $P$. aeruginosa colonization status was not deemed feasible due to its low presence at ICU admission. To align with upcoming intervention trials, but also for feasibility reasons, no quantification of the causative pathogen was included in the definition for SA ICU pneumonia (SAIP).

As described in chapter 6, this study ultimately enrolled 1,997 ICU patients in eleven European countries, 1,007 (50.4\%) of which were SA colonized and 990 (49.6\%) noncolonized at ICU admission. All were followed up during ICU stay for occurrence of protocol pneumonia, while extensive sample and data collection took place at predefined time points. The underlying source population consisted of 9,841 patients, of whom roughly a quarter were SA colonized at ICU admission (assessed in nose and LRT). We found an occurrence of 4.9 SAIP events per 1,000 days at risk (cumulative risk being $5.3 \%$ throughout ICU stay). This is a weighted estimate, calculated for the overarching source population, which was created using the source population's basic underlying characteristics. The only independent risk factor identified in the corresponding risk factor analysis was SA colonization status, with a multivariate cause-specific hazard ratio of 3.6 (95\% $\mathrm{Cl}$ 2.2-6.0). Differences were observed between European regions for SAIP incidence, microbiological culture frequency and risk magnitude for colonized patients. Results indicate that the endpoint definition used in ASPIRE-ICU is not as specific as anticipated, being sensitive for differences in diagnostic practices, for example. Furthermore, even though SA colonization contributes to the daily risk in all regions, it appears to do less so in participating countries in the southern region of Europe then in others, possibly because of locally higher SA endemicity and crosstransmission. Further confirmation of this is needed.

The attempt to design a tool for the identification of the population at risk for SAIP resulted in chapter $\mathbf{7}$, where we create a risk prediction model, using predictors available at ICU admission, and assess its performance. In this analysis, a model with SA colonization status, neurotrauma, and prior antibiotic use was best at identifying patients at highest risk for SAIP. Region was not taken along in this prediction model, considering that it would hamper extrapolation. However, in a sensitivity analysis 
assessing its added value for risk prediction, it was not selected using the Akaike's information criterion for variable selection. Even though the model will not be suitable for use in clinical practice, considering the suboptimal identification of all cases, this chapter does provide valuable insight in possible trial efficiency strategies for SAIP, as we demonstrate that the single most efficient enrollment criterion remains SA colonization status. Other predictors hardly have any additional value.

\section{What does it mean, what we did? - critical analysis of this thesis' contents}

All analyses presented in this thesis point towards colonization status being the most important risk factor for the development SA and PA ICU pneumonia. In the southern region however, this association (for SA) is not as evident, so generalizations should be made with care. The regional differences within Europe continue to challenge the interpretation of the results. In chapter 6 we suggest several explanations, of which for risk differences (in my opinion) the most plausible explanation would be the high SA endemicity in combination with higher cross transmission rates in specific areas, as this leads to swift acquisition of colonization after admission, and subsequent higher risks for those originally non-colonized.

On the other hand, for the regional incidence differences, the story is more complex. Here a contemplated driver for differences, aside from a contributing true difference in disease burden, could be differences in diagnostic work-up together with our (aspecific) SAIP definition. Cultures for example, when taken for non-clinical indications like surveillance, increase the likelihood of meeting the diagnostic criteria for SAIP, which increases the risk of misclassifying colonization as infection. On the other hand, patients suffering from pneumonia (among which SAIP) are also likely to undergo more diagnostic culturing related to pneumonia. In both situations there will be an association between SAIP occurrence and culture frequency; culturing leads to SAIP, and (SA)IP leads to culturing. As culture frequency differed between participating sites in ASPIREICU, both pathways could have played a role here. For example, if we compare the SAIP incidences found in chapter 2 (which included the Netherlands and Belgium) to that of the western region in ASPIRE-ICU (71\% of which are Dutch participants), we find lower incidences in the first, both for colonized and non-colonized. The hospitals compared here are presumed to be fairly homogenous in surveillance methods and chest X-ray work-up. Considering that the definition used in the first analysis included semi-quantitative culturing (less influenced by routine surveillance), this suggests an association of SAIP with culture frequency in this region in ASPIRE-ICU, as other differences (less patients on MV, time-effect) are unlikely to have this effect size. On 
the other hand, we did not see an overall association between a site's average culture rate and SAIP, neither in a simple scatterplot (Figure 1), nor in a more advanced logistic regression including correction for length of stay and country (OR for culture/day 0.76, $95 \% \mathrm{Cl} 0.20-2.91, p=0.68$ ). By taking the site's average we largely avoid the effect (per individual) of more culturing in case of pneumonia. A possible explanation for both findings could be that with increasing presence of surveillance cultures (western region), the ASPIRE-ICU study definition of SAIP overestimates disease presence, whereas in sites where cultures are presumably mostly taken upon indication (e.g. southern region), this overestimation is not as evident. However, even though we have information on surveillance protocols per site, it is tricky to draw firm conclusions regarding the relation between culture frequency and SAIP incidence, for the simple reason that it would ignore other differences in clinical practice (e.g. X-ray work-up, routine i.v. antibiotic prophylaxis) that could have an impact.

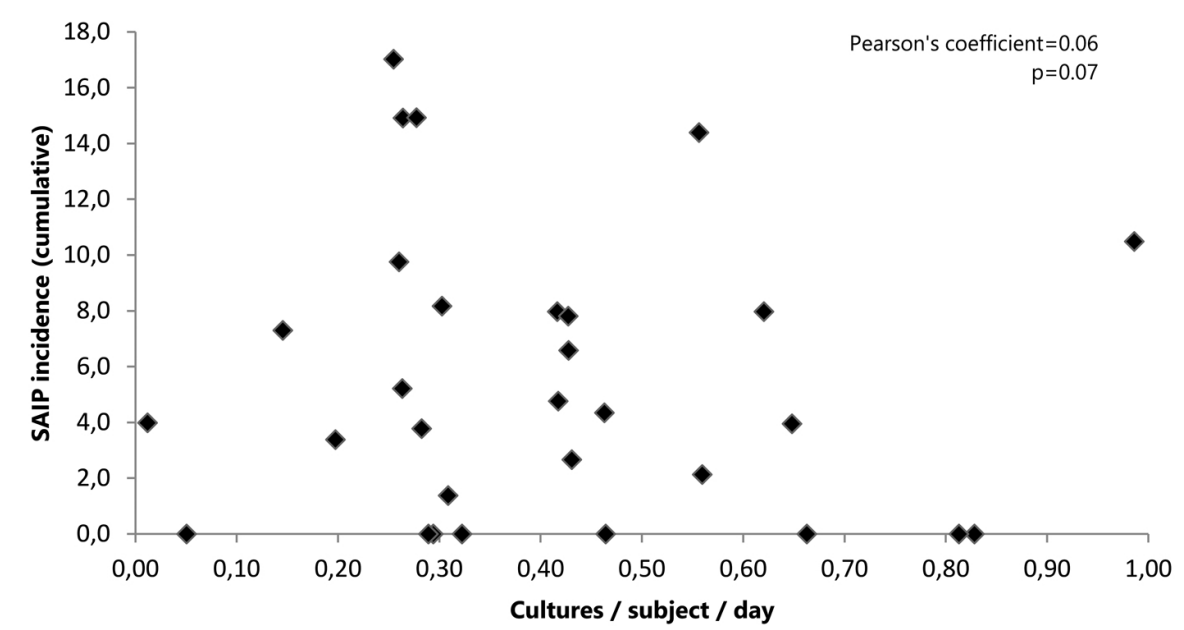

Figure 1. SAIP incidence versus average number of cultures per subject per day over the 30 sites.

Apart from the explanations and interpretations suggested earlier, another follow-up question which I couldn't help but asking myself was; could any difference in incidence or risk be the result of differences in study compliance or data collection methods? Being so closely involved in the execution of this study has provided me insight into all its 'flaws' like protocol violations, sample errors, etcetera. It crossed my mind that maybe it is a utopia to perform an international study of this size and complexity to the standard of the critical academician. Which, if indeed the case, may mean that 
other large international studies possibly find themselves in the same situation. This realization reassured and frightened me at the same time. I wondered, what does it mean, that not everything went according to plan, and could it have had an effect on our results? If so, do we know what would be the effect? To answer this question I reviewed the protocol violations, monitoring reports and I had conversations with the operational teams, to verify that data collection was (mostly) according to protocol and comparable between sites and patients (SA positive vs. negative). In this light it is worthwhile mentioning that the study design included many efforts to guarantee data quality, some of which are included in Table 1. This additional review did not reveal anything in the direction of data collection being more or less extensive for SA colonized or non-colonized (some examples in Table 2).

Table 1. Selection of ASPIRE-ICU's efforts to ensure maximum data quality

\begin{tabular}{|c|c|c|}
\hline Prior & During & After \\
\hline $\begin{array}{l}\text { Site selection using a feasibility } \\
\text { questionnaire and predefined } \\
\text { criteria (e.g. English language, } \\
\text { pneumonia work-up, capacity, } \\
\text { GCP trained) }\end{array}$ & $\begin{array}{l}\text { Remote monitoring by } \\
\text { central monitors, including } \\
\text { queries of implausible/ } \\
\text { unclear data entries. }\end{array}$ & $\begin{array}{l}\text { Thorough data cleaning, } \\
\text { including late-queries if } \\
\text { necessary. }\end{array}$ \\
\hline $\begin{array}{l}\text { Creation of working manual for } \\
\text { local team }\end{array}$ & $\begin{array}{l}\text { On site (and remote) } \\
\text { monitoring by local } \\
\text { monitors, including queries } \\
\text { of implausible/unclear data } \\
\text { entries. }\end{array}$ & $\begin{array}{l}\text { Sample verification (on- } \\
\text { site, remote and at central } \\
\text { laboratory) }\end{array}$ \\
\hline $\begin{array}{l}\text { Extensive eCRF completion } \\
\text { guidelines }\end{array}$ & $\begin{array}{l}\text { Site visits (by monitors and/ } \\
\text { or central study team) }\end{array}$ & \\
\hline $\begin{array}{l}\text { Training of study staff on study } \\
\text { protocol } \\
\text { - eLearning } \\
\text { - on site visits }\end{array}$ & $\begin{array}{l}\text { Protocol violation } \\
\text { documentation }\end{array}$ & \\
\hline \multirow[t]{3}{*}{ Run-in phase in each site } & $\begin{array}{l}\text { Re-training of staff (if } \\
\text { necessary) }\end{array}$ & \\
\hline & $\begin{array}{l}\text { Regular country calls } \\
\text { (multiple sites at same } \\
\text { time) }\end{array}$ & \\
\hline & $\begin{array}{l}\text { Newsletters, including } \\
\text { updates and reminders } \\
\text { where necessary }\end{array}$ & \\
\hline
\end{tabular}

Even though, in some sites data completeness was easier achieved than in others, it was accomplished in $>99 \%$ of the required variables. Site performance had its ups and downs, but in the end, data of 'only' 26 out of 1,997 subjects could not be 
fully monitored and verified. Protocol violations occurred, but most were minor and included for example not using the correct ICF, enrolling a non-eligible subject or missing samples. For this reason I conclude that the data are of high-quality and that the study results are valid. Thus, taking full responsibility for the ASPIRE-ICU results described in this thesis, albeit the discussed limitations and inevitable (but acceptable) glitches, I feel confident to state that, resulting from the ASPIRE-ICU data, SA colonization risk is different over different regions in Europe, even after acknowledging certain variation in diagnostic work-up. For SAIP occurrence I am slightly less confident, considering that I believe we overestimated disease presence with our definition. Here I feel that follow-up analyses using quantification on centrally analyzed samples and results on the humoral response could provide more information on the extent of overestimation of the incidence of SAIP.

Table 2. Amount of cultures and/or data completeness for SA+ versus SA-

\begin{tabular}{lll}
\hline & SA + & SA- \\
\hline Culture frequency (average n/day) & 0.45 & 0.41 \\
Missed daily pneumonia scores (\%) & 0.07 & 0.07 \\
Data unknown* (\%) & & \\
- smoking status & 29.3 & 27.4 \\
- prior antibiotic use & 10.2 & 9.2 \\
\hline
\end{tabular}

*note; the majority of the variables had $<1 \%$ missing/unknown data, here 2 variables with a reasonable amount of unknowns were used for illustration.

\section{What we would do differently, should we do it again - lessons learned}

At the start of the projects described in this thesis, we thought of many ways to do things different than before, by which we would come to the - in our eyes - long-awaited and trustworthy results which would give an answer to the question of who dunnit (in this case 'who is at risk'). Of course, some things went differently from what was expected. Firstly, we had expected to acquire many more databases for the retrospective part of the thesis, which in part is due to the methods and restriction in time period. However, this is not something that we would have done differently, as the intention was to present currently valid and representative results, instead of being fully comprehensive.

Secondly, for ASPIRE-ICU, even though we would not have chosen a different endpoint definition, considering that it was required to align with concurrent studies and that the results obtained now are very valuable, next time we would include a bacterial quantification when collecting local cultures. If we would have done this, we would 
have had more discriminative power for post-hoc analyses to separate infection from carriage. Furthermore, we would have collected the amount of chest X-rays (or other chest imaging) taken per subject per ICU stay, in order to assess if this reveals detection differences between countries/regions. If feasible it would also be worthwhile collecting for each culture/X-ray whether it was taken for surveillance purposes or because of clinical suspicion of infection.

Lastly, there are many operational lessons we have learned from performing ASPIREICU, all which were presented in a lessons learned document (unpublished, but available from the COMBACTE consortium). The most important one in my opinion, which I would encourage others to take note of, is to limit the amount of variables in the eCRF to the minimum that is needed. Having many stakeholders on board, all with different priorities towards data collection, ASPIRE-ICU ended up having an eCRF including $>100$ different possible forms per subject, slowing down the online data collection system and being very demotivating for investigators as well as for monitors. There is a difference between need-to-know and nice-to-know information, and the collected data in the 'need-to-know' section suffers in terms of quality with growing amounts of 'nice-toknow' data being asked for. Even though monitors performed source-data-verification, providing assurance on data quality in general, I do feel the quality of the collected ASPIRE-ICU data could have been higher if the requested amount had been lower.

\section{What should be done now - future perspectives}

Although this thesis ends here, it is a mere start of many of upcoming analyses as well as studies into SA and $P$. aeruginosa healthcare-associated infections. For one, the ASPIRE-ICU study will provide answers to many more research questions, apart from those already mentioned in the previous chapters. It will be able to give insight in risk factors for acquiring carriage prior to (presumed) infection, in case a patient was not a carrier at ICU admission. This may be useful in terms of infection prevention, but also in understanding transmission dynamics. Additionally, a large part of upcoming research will be focused on patients with protocol SA or PA ICU pneumonia, for example investigating differences between those colonized at ICU admission and those not. One may think of describing differences in disease course, but also finding determinants for prognosis. Especially with regard to the added value of many of the biomarkers, current expectations remain high, considering that (as indicated before) existing literature indicates prior contact with SA provides a certain level of protection in case of acquired SA infection ${ }^{17,18}$. The first results of the SAATELITE study, which reveal a preventive 
effect of pre-emptive therapy with suvratoxumab on the occurrence of SAIP (using the identical SAIP definition) also point in this direction, and are in that regard promising ${ }^{19}$.

Furthermore, results of the surgical sibling study (ASPIRE-SSI) are around the corner, bringing a lot of new insights to the table. It would be specifically interesting to see whether regional differences in overall disease risk as well as risk related to colonization status also are relevant in the surgical population. In contrast to ASPIRE-ICU, this study collected (semi-)quantitative SA screening results, which was not done to this extent before. Lastly, some of the research questions raised in this thesis, which could not be answered using data from ASPIRE-ICU, are being addressed in the intervention studies scheduled for the upcoming years. Depending on the size of these studies, they may already provide us with new insights towards differences in colonization risk per country.

I will not argue that all the suggestions in the previous paragraph are rather superficial, and describes mostly what will be done, and do not touch upon what I think potentially should or should not be done in the future, after the results of this thesis become available. With the risk of being subjective, I will shortly share my personal recommendations. In my opinion, the regional risk differences for SA colonized, as well as concerns related to endpoint definition warrant further action, considering that both could have large implications on clinical practice and/or future trials investigating pathogen specific (ICU) pneumonia. In countries with lower SA colonization risk, one can for example expect a smaller treatment effect, and a smaller effect of any quality improvement strategy involving decolonization protocols.

In addition to that, I feel that the SAIP definition used in our study is not one that is very useful to assess treatment efficacy. Not just because it overestimates disease presence, but mostly (and more importantly), because any results found in strictly regulated trials would not be generalizable to the daily clinic, where physicians take cultures and chest $X$-rays according to local standards (instead of study protocol) and use different criteria to define SAIP. Demonstration of SAIP decrease in a trial, is unlikely to be reproducible to the same extent in clinical practice, using this definition. Actually, maybe we should consider not using a pneumonia endpoint at all, but rather try to prevent nosocomial SA (or PA) infections in general, and assess efficacy through non-infectious, but objective clinical endpoints. For patients, but most likely also for treating physicians ICU mortality or duration of mechanical ventilation is more important than having SA (or PA) ICU pneumonia. Endpoints like these, being less prone to between center variability and appearing to have the support of experts in the field (if assessed in an hierarchical composite manner), may be the endpoints of the future ${ }^{20}$. 


\section{REFERENCES}

1. Williams REO, Jevons MP, Shooter RA, et al. Nasal Staphylococci and Sepsis in Hospital Patients. BMJ. 1959;2(5153):658-662. doi:10.1136/bmj.2.5153.658

2. Henderson RJ, Williams REO. Nasal Disinfection in Prevention of Post-operative Staphylococcal Infection of Wounds. BMJ. 1961;2(5248):330-333. doi:10.1136/bmj.2.5248.330

3. Nguyen MH, Kauffman CA, Goodman RP, et al. Nasal Carriage of and Infection with Staphylococcus aureus in HIV-Infected Patients. Ann Intern Med. 1999;130(3):221. doi:10.7326/0003-4819-130-3-199902020-00026

4. Sewell CM, Clarridge J, Lacke C, Weinman EJ, Young EJ. Staphylococcal nasal carriage and subsequent infection in peritoneal dialysis patients. JAMA. 1982;248(12):1493-1495. http:// www.ncbi.nlm.nih.gov/pubmed/7109173. Accessed July 15, 2019.

5. Chang FY, Singh N, Gayowski T, Drenning SD, Wagener MM, Marino IR. Staphylococcus aureus nasal colonization and association with infections in liver transplant recipients. Transplantation. 1998;65(9):1169-1172. doi:10.1097/00007890-199805150-00004

6. Chow JW, Yu VL. Staphylococcus aureus nasal carriage in hemodialysis patients. Its role in infection and approaches to prophylaxis. Arch Intern Med. 1989;149(6):1258-1262. http:// www.ncbi.nlm.nih.gov/pubmed/2658896. Accessed August 23, 2019.

7. Pujol M, Peña C, Pallares R, et al. Nosocomial Staphylococcus aureus bacteremia among nasal carriers of methicillin-resistant and methicillin-susceptible strains. Am J Med. 1996;100(5):509-516. http://www.ncbi.nlm.nih.gov/pubmed/8644762. Accessed April 10, 2014.

8. Corbella X, Domínguez MA, Pujol M, et al. Staphylococcus aureus nasal carriage as a marker for subsequent staphylococcal infections in intensive care unit patients. Eur J Clin Microbiol Infect Dis. 1997;16(5):351-357. http://www.ncbi.nlm.nih.gov/pubmed/9228474. Accessed September 8, 2014.

9. Campbell W, Hendrix E, Schwalbe R, Fattom A, Edelman R. Head-injured patients who are nasal carriers of Staphylococcus aureus are at high risk for Staphylococcus aureus pneumonia. Crit Care Med. 1999;27(4):798-801. doi:10.1097/00003246-199904000-00039

10. Rocha LA, Marques Ribas R, da Costa Darini AL, Gontijo Filho PP. Relationship between nasal colonization and ventilator-associated pneumonia and the role of the environment in transmission of Staphylococcus aureus in intensive care units. Am J Infect Control. 2013;41(12):1236-1240. doi:10.1016/j.ajic.2013.04.009 
11. Wolkewitz M, Cooper BS, Bonten MJM, Barnett AG, Schumacher M. Interpreting and comparing risks in the presence of competing events. BMJ. 2014;349:g5060. http://www. ncbi.nlm.nih.gov/pubmed/25146097. Accessed June 18, 2015.

12. Thuong M, Arvaniti K, Ruimy R, et al. Epidemiology of Pseudomonas aeruginosa and risk factors for carriage acquisition in an intensive care unit. J Hosp Infect. 2003;53(4):274-282. http://www.ncbi.nlm.nih.gov/pubmed/12660124. Accessed October 4, 2018.

13. Harris AD, Jackson SS, Robinson G, et al. Pseudomonas aeruginosa Colonization in the Intensive Care Unit: Prevalence, Risk Factors, and Clinical Outcomes. Infect Control Hosp Epidemiol. 2016;37(05):544-548. doi:10.1017/ice.2015.346

14. Berthelot P, Grattard F, Mahul P, et al. Prospective study of nosocomial colonization and infection due to Pseudomonas aeruginosa in mechanically ventilated patients. Intensive Care Med. 2001;27(3):503-512. doi:10.1007/s001340100870

15. Gómez-Zorrilla S, Camoez M, Tubau F, et al. Prospective observational study of prior rectal colonization status as a predictor for subsequent development of Pseudomonas aeruginosa clinical infections. Antimicrob Agents Chemother. 2015;59(9):5213-5219. doi:10.1128/ AAC.04636-14

16. Fowler VG, Allen KB, Moreira ED, et al. Effect of an investigational vaccine for preventing Staphylococcus aureus infections after cardiothoracic surgery: a randomized trial. JAMA. 2013;309(13):1368-1378. doi:10.1001/jama.2013.3010

17. Colque-Navarro $P$, Söderquist $B$, Holmberg H, Blomqvist L, Olcén $P$, Möllby R. Antibody response in Staphylococcus aureus septicaemia--a prospective study. J Med Microbiol. 1998;47(3):217-225. http://www.ncbi.nlm.nih.gov/pubmed/9511827. Accessed July 23, 2014.

18. Jacobsson G, Colque-Navarro P, Gustafsson E, Andersson R, Möllby R. Antibody responses in patients with invasive Staphylococcus aureus infections. Eur J Clin Microbiol Infect Dis. 2010;29(6):715-725. doi:10.1007/s10096-010-0919-x

19. François B, Garcia Sanchez M, Eggimann P, et al. Efficacy and Safety Profile of Suvratoxumab, a Novel Anti-Staphylococcus aureus Monoclonal Antibody: Results of the SAATELLITE Study in Mechanically Ventilated Intensive Care Unit Patients. In: 29th European Congress of Clinical Microbiology \& Infectious Diseases, Amsterdam. ; 2019.

20. Weiss E, Zahar J-R, Alder J, et al. Elaboration of Consensus Clinical Endpoints to Evaluate Antimicrobial Treatment Efficacy in Future Hospital-acquired/Ventilator-associated Bacterial Pneumonia Clinical Trials. Clin Infect Dis. February 2019. doi:10.1093/cid/ciz093 


$$
\pi \pi
$$


Appendix

CHAPTER

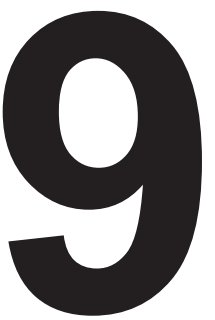




\section{SUMMARY}

Bacteria carried in or on the human body can be essential, harmless, and in some cases potentially dangerous. This thesis discusses two colonizing bacteria which both have the capability to be an innocent bystander as well as an opportunistic pathogen, which are Staphylococcus aureus and Pseudomonas aeruginosa. Especially in people who are critically ill (e.g. on an intensive care unit) or in case of skin defects (e.g. after surgery), these resident bacteria can be a threat to our health. They can, among other things, cause pneumonia or surgical site infections (the latter mainly being $S$. aureus related). It is not always clear at the start which bacterium will cause problematic infections, nor in which patient this will happen. However, we would need to know this if we want to give the patients at risk medication that can prevent it from happening.

In chapter $\mathbf{2}$ we analyzed previously collected data from two studies on intensive care unit (ICU) patients, for the occurrence of ICU acquired pneumonia caused by $S$. aureus. In the analyses we included all patients with a length of stay in ICU of at least 48 hours, and we accounted for the competing events of ICU discharge alive and death within ICU without S. aureus ICU pneumonia. The most important risk factor that we meant to quantify in this analysis was $S$. aureus colonization status, measured on the day of ICU admission or shortly before or after. A secondary analysis in this chapter investigated the occurrence of $S$. aureus ventilator-associated pneumonia (VAP), using only the patients who had been on mechanical ventilation (MV).

We found that colonization status was assessed in $58 \%$ and $80 \%$ of the cases, mainly in lower respiratory tract, and that it was S. aureus positive in $12.7-12.8 \%$. S. aureus ICU pneumonia occurred in 1.1-1.3\% of the patients, with $S$. aureus colonization at ICU admission being the most important risk factor for its development. Performing a Fine and Gray competing risks analysis we found a subdistribution hazard ratio (SHR) of 9.614.5 for colonized vs. non-colonized patients. This means that patients who are carrying S. aureus at ICU admission have a daily risk of developing $S$. aureus ICU pneumonia that is on average 9.6-14.5 times higher than patients who are known not to be colonized. The most important other risk factor that was found was MV at ICU admission, with a SHR of 3.7-7. The results of the VAP analysis were comparable, with S. aureus VAP occurring in $1.1-1.4 \%$ of the ventilated patients during ICU stay, and colonization status being associated with a SHR of 8.2-15.0.

Chapter 3 describes the development of a risk prediction model for prediction of $S$. aureus surgical site infection (SSI) within the first 90 days after undergoing cardiothoracic 
surgery. For the analysis we used the data of a randomized controlled trial that investigated the effect of a vaccine against $S$. aureus. Considering that the vaccine had no effect on $S$. aureus SSI we decided to include the complete study population in our analysis. This valuable database contained much information, including $S$. aureus colonization status before surgery. Prior to analysis we chose to investigate the following risk factors for their value in a prediction model: S. aureus colonization status, pre-operative antibiotic use, diabetes mellitus, type of cardiothoracic procedure, body mass index (BMI), age, and sex. We made this choice based on literature and clinical reasoning. We found that prior $S$. aureus colonization was the main independent risk factor for the development of S. aureus SSI after surgery, with an odds ratio of 3.1. Other independent risk factors were BMI, diabetes mellitus and type of procedure (undergoing coronary artery bypass grafting being associated with a higher risk compared to other procedures). The risk prediction model with these variables performed satisfactorily in its prediction and remained stable after internal validation with bootstrapping. However, we deemed this model not to be suitable for use in clinical practice, as less than $3 \%$ of the patients had a risk of more than $10 \%$ to develop the outcome, which results from the low occurrence of S. aureus SSI (only $2.1 \%$ ).

The research results presented in chapter 4 address the occurrence of $P$. aeruginosa ICU pneumonia, using data of one of the hospitals that was also used for the analysis in chapter 2. One of the hospitals was excluded from the analysis, because they systematically used specific medication (selective decontamination of the digestive tract) that was expected to greatly decrease the occurrence of $P$. aeruginosa ICU pneumonia. In the analysis we found that, similar as for $S$. aureus, the occurrence of $P$. aeruginosa ICU pneumonia was rather low, being $1.3 \%$ throughout ICU stay. $P$. aeruginosa colonization at ICU admission, present in $9.2 \%$ of those tested, was associated with a SHR of 8.8 compared to non-colonized patients, after accounting for competing events. Again, MV at ICU admission was also associated with a higher occurrence (SHR was 5.3).

In chapter $\mathbf{5}$ the study protocol of ASPIRE-ICU (Advanced understanding of Staphylococcus aureus and Pseudomonas aeruginosa Infections in EuRopE - Intensive Care Units) is summarized. This international prospective study was designed to create an optimal assessment of the population at risk of ICU pneumonia caused by $S$. aureus or $P$. aeruginosa, including extensive in-depth analyses into pathogen and host biomarkers. The low occurrence of $S$. aureus ICU pneumonia described in chapter 2 , has led to base the enrollment of patients for ASPIRE-ICU on the two most important risk factors identified there; $S$. aureus colonization status and mechanical ventilation 
at ICU admission. Consequently, in ASPIRE-ICU we aimed to enroll 2,000 patients on mechanical ventilation at ICU admission or (expected to be) shortly thereafter, of which $50 \%$ SA colonized and 50\% non-colonized. Considering that mechanical ventilation at ICU admission was also found to be a risk factor for $P$. aeruginosa ICU admission, this enrichment strategy was also thought to increase the rate of pneumonia caused by this pathogen. This enrichment is important, because more events make it possible to assess more risk factors in the final analysis. Unfortunately, enrichment based on $P$. aeruginosa colonization status was not feasible due to its low presence at ICU admission.

In all enrolled patients extensive data and sample collection took place, among which information on the risk factors we were interested in and daily assessment of occurrence of ICU pneumonia. The categorization of ICU pneumonia to be S. aureus and/or $P$. aeruginosa was a debated one from the start, being categorized as such also in case of detection of multiple pathogens or in case of low bacterial loads. However, this definition was chosen to align with upcoming trial definitions of intervention studies. If these definitions would differ, results could not be used to aid the trial design.

Chapter 6 discusses the analysis regarding the primary objective of ASPIRE-ICU, which is to assess the occurrence of $S$. aureus ICU pneumonia and to relate it to the risk factors that were collected. For the rationale and design see the previous paragraph and chapter 5. In total, we obtained information on a source population of 9,841 patients, of which approximately $25 \%$ were colonized with S. aureus on ICU admission. Of 1,997 patients we obtained consent to collect more elaborate data and samples, and information on whether they developed ICU pneumonia during ICU stay. For the analysis on S. aureus ICU pneumonia we were able to use information of 1,933 included subjects. This, in combination with the information retrieved from the source population led to a calculated weighted occurrence of 4.9 events of $S$. aureus ICU pneumonia per 1,000 ICU days. This estimate is a weighted estimate for the complete underlying source population. This means that for patients who did not participate in the intensive part of the study (mostly non-colonized) an estimation was made on the occurrence of $S$. aureus ICU pneumonia in them, based on basic information of them in combination with what was known for those who did participate. We saw that S. aureus ICU pneumonia occurred approximately 3.6 times more frequently in S. aureus colonized patients than in non-colonized. Apart from this we found that the occurrence was varying between European regions, as was the risk for the colonized patients. We did not find any other independent risk factors for $S$. aureus ICU pneumonia. 
In chapter 7 we use data from ASPIRE-ICU to create a risk prediction model for $S$. aureus ICU pneumonia, using information available at ICU admission. In this analysis, a model with SA colonization status, neurotrauma, and prior antibiotic use was best at identifying patients at highest risk for SAIP. We did not include the effect of region, as implementation of a model including region was not deemed feasible. However, in a sensitivity analysis assessing its added value for risk prediction, it was not selected as a relevant predictor. This chapter finishes off with insights in possible trial efficiency strategies for $S$. aureus ICU pneumonia, demonstrating that the most efficient enrollment criterion remains SA colonization status. Other predictors hardly have any additional value. 


\section{NEDERLANDSE SAMENVATTING}

De bacteriën die we bij ons dragen in of op ons lichaam kunnen zowel essentieel zijn als wel onschuldig of in sommige gevallen potentieel gevaarlijk. Dit proefschrift bespreekt twee koloniserende bacteriën, welke beiden beschikken over de capaciteit om een onschuldige toeschouwer te zijn als wel een opportunistisch pathogeen, namelijk Staphylococcus aureus en Pseudomonas aeruginosa. Vooral bij mensen die ernstig ziek zijn (bijvoorbeeld op een intensive care) of bij huiddefecten (bijvoorbeeld na een operatie), kunnen deze 'inwoners' een bedreiging zijn voor de gezondheid. Ze kunnen onder andere longontsteking (pneumonie) of post-operatieve wondinfecties veroorzaken (de laatste wordt vooral gezien bij S. aureus). Het is niet altijd vooraf duidelijk welke bacterie infecties gaat veroorzaken, of in welke patiënt. Echter, dit is wel iets wat we zouden willen weten, om zodoende preventieve medicatie te geven aan deze groep mensen, en te zorgen dat deze infecties voorkomen kunnen worden.

In hoofdstuk 2 analyseren we data van intensive care (IC) patiënten, welke eerder al (in twee aparte studies) is verzameld, op het voorkomen van op de IC verworven longontsteking veroorzaakt door S. aureus. Voor deze analyse gebruikten we data van alle patiënten die ten minste 48 uur of langer op de IC lagen en hielden we rekening met concurrerende gebeurtenissen ('competing events'), wat in dit geval levend dan wel overleden ontslag van de IC was, zonder S. aureus IC pneumonie. De belangrijkste risicofactor die we in deze analyse wilden kwantificeren was de $S$. aureus kolonisatiestatus, gemeten bij opname op de IC of kort ervoor of erna. In een secundaire analyse in dit hoofdstuk onderzochten we het voorkomen van beademingsgeassocieerde longontsteking veroorzaakt door S. aureus, waarbij we enkel de patiënten gebruikten die ooit beademd werden. Het bleek dat kolonisatiestatus gemeten was bij $58 \%$ en $80 \%$ van de patiënten, voornamelijk in de lagere luchtwegen, en dat de uitslag in $12.7-12.8 \%$ positief was voor S. aureus. S. aureus IC longontsteking trad op bij 1.1-1.3\% van de patiënten, waarbij $S$. aureus kolonisatiestatus de belangrijkste risicofactor bleek te zijn. Na het uitvoeren van een Fine en Gray concurrerende gebeurtenissen analyse ('competing events analysis') vonden we een subdistributie hazard ratio (SHR) van 9.614.5 voor gekoloniseerde vs. niet-gekoloniseerde patiënten. Dit betekent dat patiënten die drager zijn van $S$. aureus bij IC opname een dagelijks risico op het ontwikkelen van S. aureus IC pneumonie hebben dat gemiddeld genomen 9.6-14.5 maal hoger is dan patiënten waarvan we weten dat zij niet gekoloniseerd zijn. De belangrijkste andere risicofactor die we konden vaststellen was mechanische beademing bij IC opname, met een SHR van 3.7-7. In de beademingspneumonie analyse vonden we vergelijkbare 
resultaten, namelijk dat S. aureus beademingspneumonie trad op bij 1.1-1.4\% van de beademde patiënten gedurende IC opname, en dat kolonisatiestatus was geassocieerd met een SHR van 8.2-15.0.

Hoofdstuk 3 beschrijft de ontwikkeling van een risico predictie model voor het voorspellen van S. aureus post-operatieve wondinfectie (POWI) in de eerste 90 dagen na het ondergaan van cardiothoracale chirurgie. Voor deze analyse gebruikten we data uit een gerandomiseerde studie die keek naar het effect van een $S$. aureus vaccinatie. Aangezien dat dit vaccin geen effect had op de uitkomst $S$. aureus POWI gebruikten we de gehele studiepopulatie voor onze analyse. Deze waardevolle database bevatte veel gegevens, waaronder S. aureus kolonisatiestatus voorafgaand aan de operatie. Voordat we de analyse uitvoerden kozen we de risicofactoren uit waarvan we de waarde in een risico predictie model wilden onderzoeken. Dit deden we op basis van literatuur en klinisch redeneren en resulteerde in inclusie van de volgende risicofactoren; $S$. aureus kolonisatiestatus, pre-operatief gebruik van antibiotica, diabetes mellitus, type cardiothoracale procedure, body mass index (BMI), leeftijd en geslacht. We zagen dat kolonisatiestatus de meest belangrijke onafhankelijke risicofactor was voor het ontwikkelen van S. aureus POWI na operatie, met een odds ratio van 3.1. Andere onafhankelijke risicofactoren waren BMI, diabetes mellitus, en procedure type (waarbij het ondergaan van een coronaire bypass transplantatie geassocieerd was met een hoger risico dan andere procedures). Het ontwikkelde risico predictie model met deze variabelen presteerde voldoende qua predictie en bleef dat doen na interne validatie middels bootstrapping. Echter, het model is niet praktisch bruikbaar in zijn huidige vorm, gezien het feit dat minder dan 3\% van de patiënten een risico had van meer dan $10 \%$ op de uitkomst, wat voortvloeit uit het feit dat S. aureus POWI slechts weinig voorkwam (2.1\% in totaal).

De onderzoeksresultaten die gepresenteerd worden in hoofdstuk 4 richten zich op het voorkomen van $P$. aeruginosa IC longontsteking, en beschrijven data van één van de ziekenhuizen die ook gebruikt is voor de analyse van hoofdstuk 2 . Eén van de ziekenhuizen werd niet meegenomen in deze analyse, omdat er daar gebruik werd maakt van bepaalde medicatie (selectieve darm decontaminatie) die het voorkomen van $P$. aeruginosa IC longontsteking naar verwachting sterk doet afnemen. Net als bij S. aureus zagen we dat het voorkomen van $P$. aeruginosa IC pneumonie relatief zeldzaam was, met een optreden bij $1.3 \%$ van de patiënten gedurende de IC opname. $P$. aeruginosa kolonisatie bij IC opname, aanwezig bij 9.2\% van de patiënten die hiervoor waren getest, was geassocieerd met een SHR van 8.8 in vergelijking tot niet gekoloniseerde patiënten, na het rekening houden met concurrerende gebeurtenissen. Opnieuw was mechanische 
beademing bij IC opname ook geassocieerd met een toegenomen voorkomen (de SHR was 5.3).

In hoofdstuk 5 wordt het studie protocol van ASPIRE-ICU (Advanced understanding of Staphylococcus aureus and Pseudomonas aeruginosa Infections in EuRopE - Intensive Care Units, ofwel 'Beter begrijpen van Staphylococcus aureus en $P$. aeruginosa infecties in Europa - Intensive Care Units') samengevat. Deze internationale en prospectieve studie is vanaf het begin af aan ontworpen om een optimale database te creëeren voor het vaststellen van de groep patiënten die het grootste risico lopen op een $S$. aureus of $P$. aeruginosa IC longontsteking, inclusief uitgebreide, diepgaande analyses naar pathogeen en gastheer-gerelateerde biomarkers. De lage incidentie (voorkomen) van S. aureus IC longontsteking, beschreven in hoofdstuk twee, leidde tot het selecteren van patiënten voor deze studie op basis van de twee belangrijkste risicofactoren gevonden in hetzelfde hoofdstuk, namelijk S. aureus kolonisatiestatus en mechanische beademing. Hieruit volgend includeerden we een studiepopulatie die voor de helft bestond uit $S$. aureus gekoloniseerden en voor de helft uit niet gekoloniseerden. Daarnaast moest iedereen mechanisch beademd worden bij opname (of kort erna), om te kunnen deelnemen. Aangezien mechanische beademing ook als risicofactor voor $P$. aeruginosa IC pneumonie werd aangetoond verwachtten we dat deze strategie ook zou zorgen voor een hoger aantal pneumoniën veroorzaakt door deze bacterie. Een hoger aantal pneumoniën is belangrijk, want meer 'uitkomsten' maken het mogelijk om meer risicofactoren te testen in de uiteindelijke analyse. Om die reden is het jammer dat we verdere verrijking o.b.v. $P$. aeruginosa kolonisatie niet haalbaar achtten, gezien het lage voorkomen ervan bij patiënten bij IC opname. Bij alle geïncludeerde patiënten vond uitgebreide data en monsterafname plaats, en zodoende verzamelden we alle benodigde informatie ten aanzien van risicofactoren of de patiënt een longontsteking had ontwikkeld. Het categoriseren van IC pneumonie als zijnde veroorzaakt door $S$. aureus en/of $P$. aeruginosa was een intensief bediscussieerd onderwerp vanaf het begin, aangezien onze studie het ook een dergelijke pneumonie noemt als er meerdere soorten bacteriën aanwezig zijn of als de hoeveelheden zeer minimaal zijn. Deze definitie is echter gekozen in afstemming met aankomende medicijn-studies, en hun definities van deze pneumoniën. Als we andere definities gekozen zouden hebben dan zouden onze resultaten niet gebruikt kunnen worden voor het ontwikkelen van een toekomstige studieopzet.

Hoofdstuk 6 bespreekt de analyse van de primaire uitkomst van ASPIRE-ICU, namelijk het voorkomen (de incidentie) van S. aureus IC pneumonie. Daarnaast wordt er een eerste risicofactor analyse gedaan. De achtergrond en opzet van de studie zijn 
reeds besproken in de vorige paragraaf (over hoofdstuk 5). In totaal verkregen we informatie van een bronpopulatie van 9,841 patiënten, waarvan ongeveer $25 \%$ S. aureus gekoloniseerd was bij IC opname. Van in totaal 1,997 patiënten kregen we toestemming voor uitgebreide data- en monsterverzameling en werd ook vastgesteld of ze IC pneumonie ontwikkelden. Voor de analyse van S. aureus IC pneumonie konden we de gegevens van 1,933 gebruiken. Dit in combinatie met de gegevens van de bronpopulatie, bracht ons tot een gewogen incidentie van S. aureus IC pneumonie van 4.9 per 1,000 dagen op de IC. Dit getal is een gewogen incidentie voor de gehele onderliggende bronpopulatie op de IC. Dit betekent dat voor de patiënten die niet meededen met het intensieve deel van de studie (voornamelijk niet-gekoloniseerden), een schatting is gedaan van het optreden bij hen, op basis wat bekend was bij de patiënten die wel meededen. Bij S. aureus gekoloniseerde patiënten kwam grofweg 3,5 keer vaker $S$. aureus IC pneumonie voor dan bij niet gekoloniseerden. Daarnaast zagen we dat in de verschillende Europese regio's het voorkomen verschillend was, en evenals het risico voor gekoloniseerden. We vonden geen andere onafhankelijke risicofactoren in deze risico-analyse.

In hoofdstuk 7 hebben we data uit ASPIRE-ICU gebruikt om een risico predictie model te maken dat het optreden van $S$. aureus IC pneumonie kan voorspellen, gebruik makend van gegevens die beschikbaar zijn op het moment van IC opname. In deze analyse zagen we dat een model met daarin uitslag van kolonizatiestatus, wel/geen neurotrauma en wel/niet vooraf gebruik van antibiotica het beste was in het voorspellen of een patiënt gedurende zijn opname $S$. aureus IC pneumonie zou krijgen. In dit model hebben we niet gekeken naar het effect van regio, aangezien de uitslagen dan niet toepasbaar zou zijn buiten de landen die mee hebben gedaan aan ASPIRE-ICU. Wel hebben we gecheckt of dit grote invloed heeft gehad op de uitslagen, door middel van een sensitiviteitsanalyse, waarbij we regio wel includeerden. Dit had geen effect. Dit hoofdstuk sluit af met inzichten t.a.v. verhogen van trial efficiëntie, waarbij we laten zien dat kolonizatiestatus de meest waardevolle en efficiëntste selectiecriterium zou zijn in een volgende medicijnstudie naar S. aureus IC pneumonie. De andere voorspellers hadden nauwelijks toegevoegde waarde meer. 


\section{DANKWOORD}

Het zit erop, eindelijk $($ ). Hierbij een paar woorden van dank, aan de mensen die hebben bijgedragen aan dit proefschrift.

Prof. Kluytmans, beste Jan, hoewel niet vanaf het begin mijn promotor, wel mijn eerste, en de eerste plek hier is dan ook meer dan vanzelfsprekend. Toen jij 'aanschoof' was Stage 1, maar ook de ontwikkeling van ASPIRE al enige maanden gaande. Eigenlijk heel snel al kreeg ik echt het gevoel dat we dit project 'samen' deden, en dat gevoel is niet meer weggetrokken. Zelfs al had je vaak maar beperkt de tijd, of waren de omstandigheden moeilijk, je was nagenoeg altijd bereikbaar, luisterde, en dacht mee. Ook als er geen oplossingen waren in mijn ogen, dan had jij er wel eentje, en kwamen we er samen toch uit. Gezien de hobbels die we in deze jaren zijn tegengekomen, op meerdere vlakken, was dit behalve waardevol voor mij ook heel bijzonder. Naast dat ik je uiteraard wil bedanken voor je wetenschappelijke steun en bijdragen, bedankt voor dit team-gevoel.

Prof. Bonten, beste Marc, ik weet nog dat ik hier begonnen ben en dat jij zei dat er twee soorten promotietrajecten bestaan. Beide versies begonnen met een sprong in een zwembad, waarbij je bij het ene min of meer binnen de lijntjes de afgesproken baantjes moest trekken om (op tijd) bij het einde van je promotie aan te komen. (Je klonk niet echt laaiend hierover.) Dan was er nog het andere traject, waarbij je zonder zwemdiploma in het diepe werd gegooid en zelf de koers moest bepalen naar een nog onbekend doel. Als ik het zo opschrijf weet ik niet waarom, maar je maakte me enthousiast voor dat laatste. Eigen verantwoordelijkheid, eigen invulling, hands-on onderzoek van begin tot eind, dat klonk als een leuke uitdaging. Op dat moment wisten we allebei (gelukkig) niet dat het project waar ik uiteindelijk terecht gekomen ben een grotere kluif was dan de bedoeling was, maar de beschrijving van het diepe zwembad klopte wel. Hier heb ik veel van geleerd en ik wil je graag bedanken voor deze kans die je me hebt gegeven, het vertrouwen dat je in me hebt gelegd en je inspanningen om tot een mooi eindproduct te komen.

Leden van de beoordelingscommissie, prof. van Strijp, prof. de Smet, prof. Heederik, prof. Girbes, prof. Vandenbroucke-Grauls, dank voor het nemen van de tijd om mijn proefschrift te beoordelen.

Dear Freiburg-team; Susanne, Kristin, Derek, and of course Martin; the quality of the analyses would not have been of this standard if it was not for you. Apart from that, I always liked going to Freiburg, or having you over in Utrecht. Too bad that finishing 
my thesis, is a competing event for future visits to Freiburg, as the daily risk to have our face-to-face meetings clearly drops after this day has passed. You are more than welcome in Houten at any time, should you be around.

Universiteit Antwerpen; prof. Goossens, prof. Malhotra-Kumar, Leen, Christine, Jasmine, en andere betrokken collega's; bedankt voor de onmisbare laboratorium gerelateerde input gedurende zowel de ontwikkeling, als uitvoer, als analyse en opschrijven van ASPIRE-ICU. Naast dat ik hier veel van heb geleerd zorgde dit er ook voor dat de participerende ziekenhuizen wisten wat ze moesten doen en werden alle (of in ieder geval zoveel mogelijk :-S) samples op tijd afgenomen, ingevroren, vervoerd en geanalyseerd. Maar het belangrijkste blijft uiteraard dat we door onze samenwerking tot mooie gezamenlijke resultaten konden komen.

Medlmmune team (I know it is AstraZeneca now, but for me it will be Medlmmune I guess for a while still), thank you for your support on all fronts. While risking forgetting someone, I want to mention some people in particular. Firstly and foremost; Frank Sifakis, the 'father' of the projects in this thesis, you were there from the start and even before, and you have been part of every step and hurdle that was taken. Even though you are not part of AZ anymore, I hope you are OK with me addressing you here. I respect your ability to always find solutions for difficult issues, articulate delicate matters in a calm way, and to keep making jokes even in the most unpleasant and unfunny situations. Mark Eickhoff; although you were away from the project even before the ASPIRE recruitment had started, without your eye for detail we would not have had the same quality of protocol as we had now. Rubana; the difficult task of taking over from Mark did not seem to bring you problems. You may have even brought more perfection to the study as a whole, and in particular the development of the eCRF and study site selection. Thank you, your perseverance is admirable. Lastly; Omar, Alexey, Kathryn, Hasan, Michael and all that I now do not mention by name, thank you for your input and guidance where needed.

All investigators in the 30 sites of ASPIRE-ICU, including the principal investigators, subinvestigators, research nurses, laboratory technicians, clinical trial assistants, monitors; thank you for your efforts and dedication to the execution of this study. I know it was not simple, and no fun at many occasions. Your enthusiasm was much appreciated as well as needed.

All co-authors, mentioned and non-mentioned, thank you for your valuable contributions. Karina, it was a pleasure to work with you during your year here (and to learn some Norwegian words and customsJ). 
COMBACTE-team, ik weet dat deze studie er hoe dan ook geweest zou zijn, ook zonder mij. Toch kon ik het me ook eigen maken, bedankt voor deze mogelijkheid. Claire-Marie; van de early hours, altijd gezellig even binnen te lopen. Ik zal je opgewektheid missen. Nienke, bedankt voor je bijdrage aan de site selectie.

Het ASPIRE-ICU studie management team in Utrecht; Rianne, Jelle, Nicolette, Janet, Elien, Leo, Bas, Daniël; het lijkt me duidelijk, zonder jullie inzet voor de sites was er überhaupt geen ASPIRE-ICU studie geweest. Elien; jouw inzet en continuïteit heeft voor veel verbetering van de kwaliteit en betrouwbaarheid van de data gezorgd, op veel sites. Zeker na Daniëls vertrek was dit van grote waarde! Rianne, Jelle, Nicolette, jullie kwamen er later bij, maar er was genoeg te doen, samen konden jullie het einde van de studie, en de drukte die kwam kijken bij het data cleanen en het sluiten van de sites goed aan. Janet, je had altijd het overzicht. Leo, ook jij bedankt voor je bijdragen.

Datamanagers; Chantal, Jildou, Sandor; ASPIRE-ICU is pittig geweest, en ondanks dat bleef jullie inzet gewaarborgd. Bedankt dat jullie onze verzoekjes altijd serieus namen en dat we vaak snel tot een oplossing van de problemen konden komen. Jildou, ik ben jaloers op de fantastische trips die je maakt. Hopelijk kan ik zijdelings af en toe nog wat meekrijgen van je belevenissen! Maar ook bedankt voor je eerlijke aanwezigheid in de tweede helft van de studie, het heeft veel van je gevraagd. Sandor, zoals ik ook tegen jou heb gezegd, ik weet onze eerste afspraak nog. Allebei totaal bleu wat betreft ASPIRE en de omvang ervan. Ik heb het idee dat jouw verbondenheid met het project (die toen nog niet echt aanwezig was, begrijpelijkerwijs), nu bijna net zo groot is als mijn eigen verbondenheid ermee. Je verantwoordelijkheidsgevoel, drive om het op de juiste manier aan te leveren, maar ook te controleren wat je aanlevert is (naast erg tijdrovend voor jezelf) bewonderenswaardig. Je bent misschien wel niet zo'n dwarsligger als je denkt ;-).

Bas, Daniël; jemig wat een klus. Wisten jullie waar je aan begon met ASPIRE-ICU? Soms vraag ik me dat weleens af. Maar ook weet ik dat managen jullie allebei goed ligt, en daarbij uitdaging en plezier biedt. Daar ben ik blij om, want door jullie overzicht, daadkracht, helderheid en structuur kunnen we dit project op een goede manier afsluiten. Of zoals jij het weleens hebt gezegd Bas: kunnen we de verantwoordelijkheid voor de data met vertrouwen dragen. Zo voelt het voor mij ook, en dat blijkt nog meer uit het feit dat jullie bereid waren om op deze dag achter en naast mij te staan. Mijn dank is groot. 
Huisartsenpraktijk Mondriaanlaan; Johan \& Erik, ik weet dat (de druk van) het proefschrift voelbaar was in Nieuwegein. Dank voor de ruimte die ik heb gekregen om het goed af te ronden.

Kamer 5.143; Karlijn, Marlous, Wouter, Eva, Douwe, Henri, Alwin, Sabine, Martine, Lisa, Indira, Shona, Gerrita, Alies, Laura; in de eerste jaren heb ik veel kamergenoten zien langskomen, en het was altijd een goede mix van gezelligheid en (af en toe (:)) werken. Als één van ons vastliep was er altijd iemand om naar je te luisteren en te helpen, of een rondje te lopen. Ik heb me er altijd thuis gevoeld. Infectie-epi's / (XE-)WMM'ers, wat een gezelligheid met de ECCMID's, post-WMM coffeebreaks, Journal Clubs en wat fijn om zulke constructieve feedback te krijgen wanneer nodig. Van-Geunsjes; het laatste jaar op het Julius was ik net als alle andere promovendi 'verbannen' naar het van Geuns. Dat vond ik helemaal niet erg, de rustige sfeer, de weekstarts en rondjes lopen maakte het tot de perfecte plek om mijn proefschrift zo ver mogelijk af te krijgen. Leuk om met zoveel tegelijk in 'hetzelfde schuitje' te zitten. Daarnaast toch even apart; Darren, Denise, toen ik veel afwezig was i.v.m. de opleiding, waren jullie er wel. Het operationele team heeft veel aan jullie steun gehad, maar ikzelf des te meer. Zonder jullie overzicht en bijdragen, maar ook spar-momenten en steun was de uitkomst van dit alles niet geworden tot wat het nu is. Tim, Lisanne, als buren/kamergenoten hebben we veel binnengelopen bij elkaar, de ene keer langer dan de andere keer. Jammer dat dit nu niet meer zo is! Claudia, even though many hurdles were already taken and many victories already celebrated, harvest time has only just begun. Your fresh perspective is most welcome, and I wish you good luck and lots of fun in the coming years.

Lieve vriendinnen; Miriam, Sjaane, Madelon, Marijn, Elsemiek, Sharon, Sophie, Willemijn, Daniëlle, Carolien, bedankt dat jullie meermaals peptalks hebben gegeven en oneindig frustraties hebben aangehoord. Voor een deel van jullie is het bekende koek, want jullie hebben hetzelfde (phd-)proces doorgemaakt. Maar ook voor degenen waar dat niet voor geldt, jullie warmte, of af en toe eens een eerlijke reflectie, was heel waardevol tijdens het hele proces. Bedankt!

Lieve (schoon)familie, een promotie is niet iets wat je alleen maar 'op je werk' doet. Veel van jullie hebben vaak het wel en wee mogen meebeleven in de afgelopen jaren. Dank dat jullie er naar zijn blijven vragen, interesse bleven tonen, en daarmee steun boden, ook op de moeilijkere momenten. Dit ondanks dat het langer duurde dan gepland, en voor het grootste deel van jullie niet de meest interessante of begrijpelijke materie is ;-). Ida en Hildebrand, bedankt dat jullie altijd voor ons klaar staan, dat voelt heel fijn. Ik prijs me gelukkig om zulke lieve schoonouders te hebben. 
Pap, bij jou kan ik altijd terecht voor goed rationeel advies. Je windt er geen doekjes om, en daar hou ik van. Bedankt voor je aanwezigheid op de momenten dat dit nodig was. Ik vind het heerlijk om te zien hoe je kunt genieten van je kleinkinderen en zij van jou. Mam, Nico, afgelopen jaren is er een hoop gebeurd en veranderd. Grootouders worden, verhuizingen, ziekte. Steun zoeken en steun bieden wisselden elkaar af. Weet dat jullie aanwezigheid en bemoediging onmisbaar zijn geweest, op zoveel momenten (en dan bedoel ik niet alleen door het oppassen). Zo fijn dat we dicht bij elkaar in de buurt wonen, en elkaar zo vaak kunnen zien.

Lief gezin, mijn twee schatjes en grootste schat. Er is niets belangrijker dan jullie. Wat heb ik jullie soms gemist als ik weer moest werken. Zeker de laatste maanden was dit helaas vaker dan we alle vier leuk vonden.

Rosalie en Maurice, bedankt dat er genoeg afleiding was als ik thuiskwam na een dag onderzoek of op de praktijk. Jullie hebben er nauwlettend voor gezorgd dat ik buiten werk zo min mogelijk met promoveren bezig was, heel goed gedaan $(;)$.

Lieve Laurens, het is klaar. Af! Wie had dat gedacht. Ik heel vaak niet, maar jij wel. Jij bent misschien wel degene die het meest van allen begrijpt wat voor een last hiermee van mijn schouders valt. Hoe vaak jij niet dingen hebt moeten aanhoren, heb ik niet geteld. Gekscherend heb je vaak geroepen dat jij wel mijn paranimf kon worden. Maar jij bent mijn steun en toeverlaat voor veel meer dan alleen mijn promotietraject en veel meer dan alleen een steun en toeverlaat. Vanaf nu is er meer ruimte voor ons, en voor onze mooie kinderen en plannen samen. Ik kan niet wachten 


\section{CURRICULUM VITAE}

Fleur was born on $28^{\text {th }}$ of August 1985 in Groningen. She lived there with her parents and brother Jules, until they moved to Haren, where she graduated from the Zernike College in 2003. After this, she travelled through South America for six months, before deciding to start Medical School in Utrecht.

During her study time she did several internships in foreign hospitals, as well as an internship specializing in infectious diseases. After graduation in 2011, she started working in Internal Medicine in the Zuwe Hofpoort ziekenhuis in Woerden, which was followed by a year in the Diakonessenhuis in Utrecht/Zeist in the same field.

As infectious diseases kept drawing her interest she started working as a PhD in the Julius Center for Marc Bonten starting January 2013. In the beginning the focus was on community acquired-pneumonia, but during the first year this shifted to ICU-acquired pneumonia. It led to being the driving force of (what would be) ASPIRE-ICU.

After realizing that the study would take longer than anticipated, she applied for the general practitioner (GP) training. Between September 2015 and September 2018 she participated in research one day per week and spent her other days in training to become a GP. Spending one last year completely on science, she was able to finalize her PhD. In the following year she will finish her third year of GP training to complete the trajectory. During her PhD Fleur gave several oral and poster presentations on conferences in Europe and completed the postgraduate Master in Clinical Epidemiology at Utrecht University.

Fleur is married to Laurens, and they live together in Houten with their two children Rosalie (2016) and Maurice (2017).

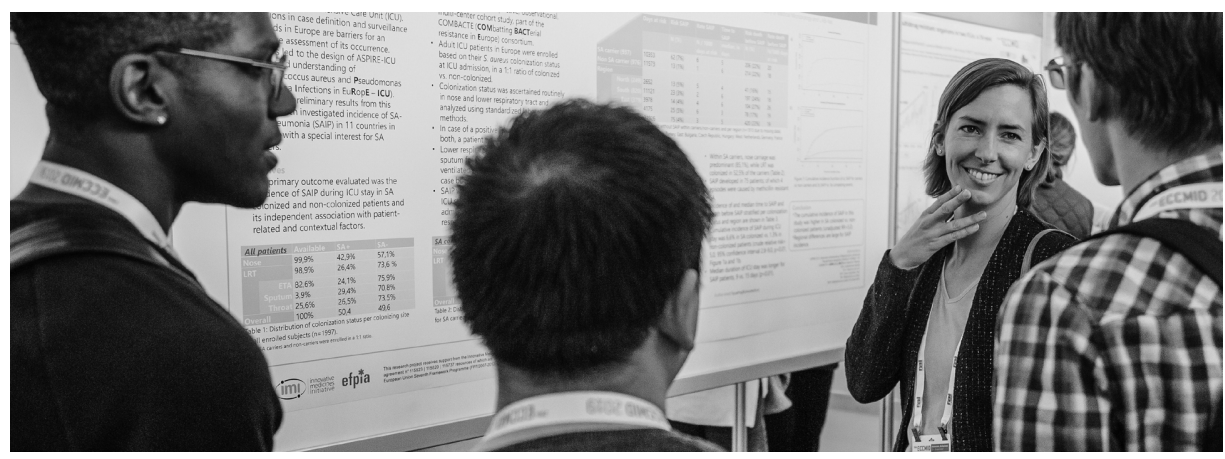




\section{LIST OF PUBLICATIONS}

\section{Publications related to this thesis}

Paling, F. P., Wolkewitz, M., Bode, L. G. M., Klein Klouwenberg, P. M. C., Ong, D. S. Y., Depuydt, P., ... Kluytmans, J. A. J. W. (2017). Staphylococcus aureus colonization at ICU admission as a risk factor for developing S. aureus ICU pneumonia. Clinical Microbiology and Infection, 23(1), 49.e9-49.e14. https://doi.org/10.1016/j.cmi.2016.09.022

Paling, F. P., Wolkewitz, M., Depuydt, P., de Bus, L., Sifakis, F., Bonten, M. J. M., \& Kluytmans, J. A. J. W. (2017). P. aeruginosa colonization at ICU admission as a risk factor for developing P. aeruginosa ICU pneumonia. Antimicrobial Resistance and Infection Control, 6(1). https://doi.org/10.1186/s13756-017-0197-9

Paling, F. P., Troeman, D. P. R., Wolkewitz, M., Kalyani, R., Prins, D. R., Weber, S., ... Kluytmans, J. A. J. W. (2017). Rationale and design of ASPIRE-ICU: A prospective cohort study on the incidence and predictors of Staphylococcus aureus and Pseudomonas aeruginosa pneumonia in the ICU. BMC Infectious Diseases, 17(1). https://doi. org/10.1186/s12879-017-2739-4

Paling, F. P., Olsen, K., Ohneberg, K., Wolkewitz, M., Fowler, V. G., DiNubile, M. J., ... Kluytmans, J. A. J. W. (2018). Risk prediction for Staphylococcus aureus surgical site infection following cardiothoracic surgery; A secondary analysis of the V710-P003 trial. PLoS ONE, 13(3). https://doi.org/10.1371/journal.pone.0193445

\section{Publications unrelated to this thesis}

Engel, M. F., Paling, F. P., Hoepelman, A. I. M., van der Meer, V., \& Oosterheert, J. J. (2012). Evaluating the evidence for the implementation of c-reactive protein measurement in adult patients with suspected lower respiratory tract infection in primary care: $A$ systematic review. Family Practice, 29(4). https://doi.org/10.1093/fampra/cmr119

Paling, F. P., Andrews, L. M., Valk, G. D., \& Blom, H. J. (2012). Life-threatening complications of ibogaine: three case reports. The Netherlands Journal of Medicine, 70(9), 422-424. Retrieved from http://www.ncbi.nlm.nih.gov/pubmed/23123541

Van Werkhoven, C. H., Huijts, S. M., Paling, F. P., \& Bonten, M. J. M. (2016). The scrutiny of identifying community-acquired pneumonia episodes quantified bias in absolute effect estimation in a population-based pneumococcal vaccination trial. Journal of Clinical Epidemiology, 69. https://doi.org/10.1016/j.jclinepi.2015.07.004 




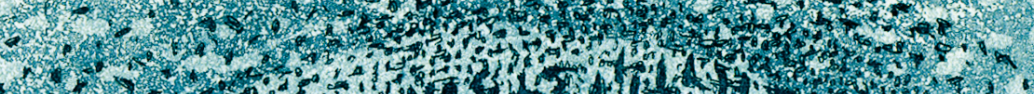

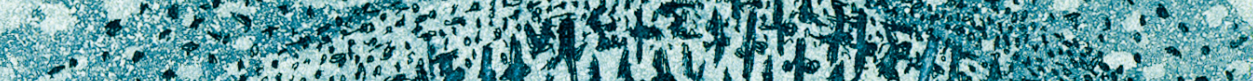

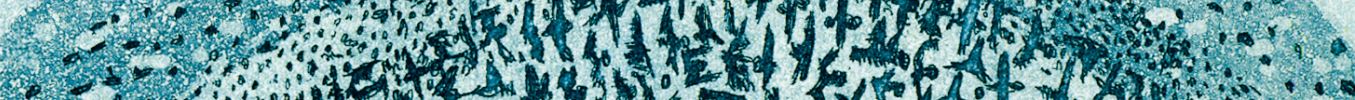

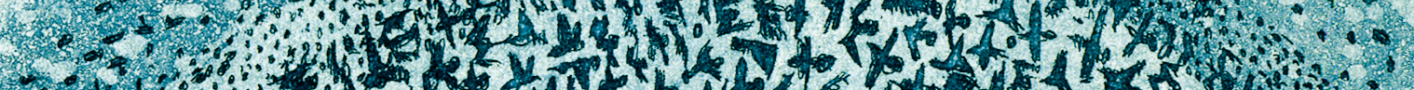
2. 20.

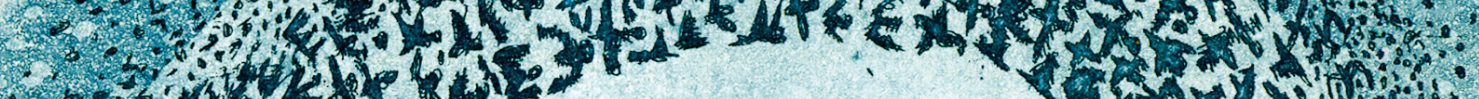

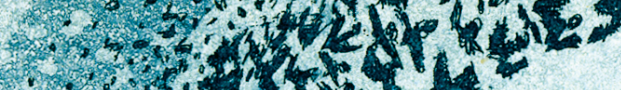

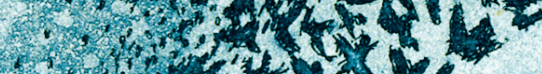
6.7. 3 .

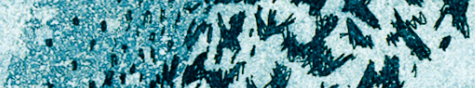

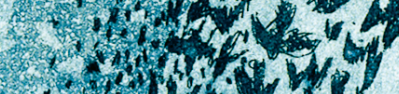

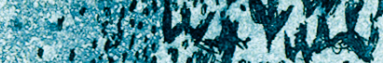

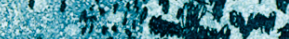

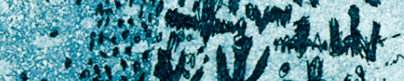
.

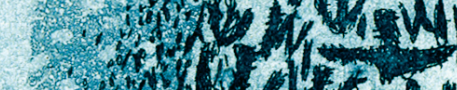
-

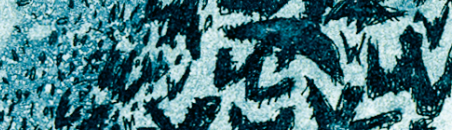

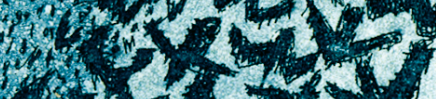
6.

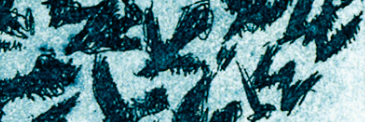

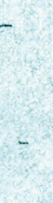

